Division of Geological \& Geophysical Surveys

RAW-DATA FILE 2007-4

\begin{abstract}
MAJOR-OXIDE, MINOR-OXIDE, TRACE-ELEMENT, GEOCHEMICAL, AND NON-CARBONATE CARBON DATA FROM ROCKS COLLECTED IN THE SOLOMON AND NOME QUADRANGLES, SEWARD PENINSULA, ALASKA IN 2006
\end{abstract}

by

Melanie B. Werdon, Rainer J. Newberry, David J. Szumigala, Larry K. Freeman, and Jennifer E. Athey

$\$ 5.00$

September 2007

THIS REPORT HAS NOT BEEN REVIEWED FOR TECHNICAL CONTENT OR FOR CONFORMITY TO THE EDITORIAL STANDARDS OF DGGS

Released by

STATE OF ALASKA

DEPARTMENT OF NATURAL RESOURCES

Division of Geological \& Geophysical Surveys 3354 College Road

Fairbanks, Alaska 99709-3707 



\section{CONTENTS}

PAGE

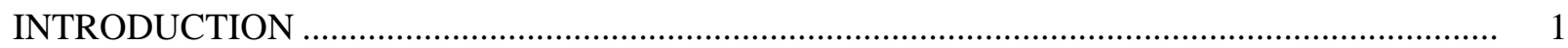

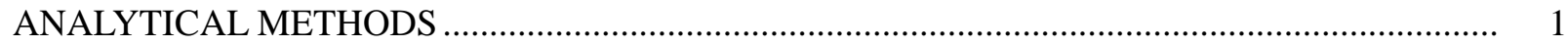

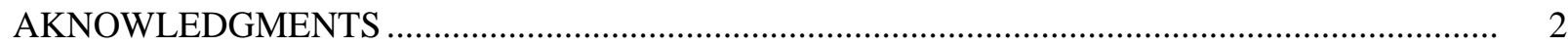

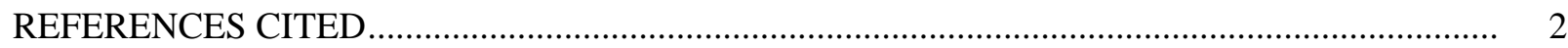

\section{TABLES}

TABLE 1. LOCATION AND DESCRIPTION OF ROCK SAMPLES COLLECTED FOR TRACEELEMENT GEOCHEMICAL ANALYSES IN THE SOLOMON QUADRANGLE, SEWARD PENINSULA, ALASKA.

TABLE 2. CONCENTRATION OF TRACE ELEMENTS IN ROCK SAMPLES COLLECTED IN THE SOLOMON QUADRANGLE, SEWARD PENINSULA, ALASKA

TABLE 3. LOCATION AND DESCRIPTION OF ROCK SAMPLES COLLECTED FOR MAJOROXIDE, MINOR-OXIDE, AND TRACE-ELEMENT ANALYSES IN THE SOLOMON AND NOME QUADRANGLES, SEWARD PENINSULA, ALASKA

TABLE 4. CONCENTRATION OF MAJOR-OXIDES, MINOR OXIDES, AND TRACE ELEMENTS IN ROCK SAMPLES COLLECTED IN THE SOLOMON AND NOME QUADRANGLES, SEWARD PENINSULA, ALASKA..

TABLE 5. DETECTION LIMITS AND ANALYTICAL METHODS FOR TRACE-ELEMENT GEOCHEMICAL ANALYSES.

TABLE 6. DETECTION LIMITS AND ANALYTICAL METHODS FOR MAJOR-OXIDE, MINOROXIDE, AND TRACE-ELEMENT ANALYSES

TABLE 7. LOCATION AND DESCRIPTION OF ROCKS COLLECTED FOR NON-CARBONATE CARBON ANALYSES IN THE SOLOMON QUADRANGLE, SEWARD PENINSULA, ALASKA [INCLUDES DETECTION LIMITS AND ANALYTICAL METHOD].

Note: This report (including all analytical data and tables) is available in digital format from the DGGS web site (http://wwwdggs.dnr.state.ak.us) at no charge. The digital data are available as PDF files and Excel spreadsheets. 



\title{
MAJOR-OXIDE, MINOR-OXIDE, TRACE-ELEMENT, GEOCHEMICAL, AND NON- CARBONATE CARBON DATA FROM ROCKS COLLECTED IN THE SOLOMON AND NOME QUADRANGLES, SEWARD PENINSULA, ALASKA IN 2006
}

\author{
by \\ MELANIE B. WERDON, RAINER J. NEWBERRY, DAVID J. SZUMIGALA, LARRY K. \\ FREEMAN, and JENNIFER E. ATHEY
}

\section{INTRODUCTION}

Mineral-resource personnel from the Alaska Division of Geological \& Geophysical Surveys carried out a geological field survey, including mapping and sampling in the Solomon Quadrangle, Seward Peninsula, Alaska from June 15 to July 26, 2006, and a reconnaissance visit to the Nome Quadrangle. The fieldwork provides basic information critical to building an understanding of Alaska's geology and is part of an integrated program of airborne geophysical surveys followed by geological mapping. During 2006, 211 rock samples were collected for geochemical trace-element analysis, 24 rock samples were collected for whole rock (major- and minor-oxides, and petrogenetically important trace-elements) analysis, and 3 samples were collected for non-carbonate carbon analysis. Location coordinates were collected using a hand-held GPS unit (no differential correction was applied), and coordinates are presented in latitude and longitude (based on the NAD 27 Alaska datum) and in UTM coordinates (based on the Clark 1866 spheroid, NAD 27 datum, UTM zone 3 projection). Additional details about the sampling program can be found in the metadata file associated with the digital version of this report. Sample numbers, location data, descriptions, and analytical results for each sample are tabulated in Tables 1, 2, 3, 4, and 7.

\section{ANALYTICAL METHODS}

All trace-element geochemical analyses (table 2) collected in 2006 were performed by ALS Chemex. Rock samples were crushed with a Terminator jaw crusher with chrome steel alloy plates so that at least 70 percent of the material passed through a $-10(2 \mathrm{~mm})$ mesh screen. Representative aliquots of 250 grams and 30 grams each were taken using a stainless steel riffle splitter. These samples were then pulverized in a chrome steel ring mill so that 85 percent of the sample passed through a -200 (75 micron) mesh screen. Most trace-element analyses were performed on the 250 gram split while gold analyses were performed on the 30 gram representative split.

Initially, all samples were assayed for gold by atomic absorption spectroscopy following a fire assay fusion (FA-AAS). Samples containing greater than 10,000 ppb gold were also assayed by gravimetric finish following a fire assay fusion (FA-GRAV). All samples were assayed for mercury by inductively coupled plasma-atomic emission spectroscopy (ICP-AES) after aqua regia digestion, and by atomic absorption spectroscopy (AAS) after aqua regia digestion/cold vapor. High-grade copper samples were assayed by AAS. All other trace-element geochemical analyses were performed by inductively coupled plasma-atomic emission spectroscopy (ICP-AES) methods following aqua regia digestion. This method of digestion is possibly incomplete for some elements and may result in lower analytical results for certain elements. The elements that may be affected by incomplete digestion as well as analytical methods, and lower and upper detection limits are tabulated in Table 5. 
All whole rock analyses (table 4) were performed by ALS Chemex. Major- and minor-element oxides were determined by X-ray fluorescence spectrometry following a lithium metaborate fusion (LBF-XRF). Trace elements (Nb, Rb, Sr, Y, and $\mathrm{Zr}$ ) were determined using wavelength-dispersive $\mathrm{X}$-ray fluorescence spectrometry on pressed pellets (PP-XRF). Analytical methods and detection limits are tabulated in Table 6.

In order to check assay values reported by laboratories, DGGS inserts well-characterized rock standards throughout our sample order. Inspection of the analyses of DGGS's whole rock standards indicated that pressed-pellet XRF values for yttrium (Y) reported by ALS Chemex were too low. Upon our request, ALS Chemex examined their internal standards and stated that the Y values were within their accepted range of standard deviation. To examine this issue further, DGGS submitted the leftover pulps from basalt and gabbro samples to the University of Alaska Fairbanks (UAF) Advanced Instrumentation Laboratory for comparative XRF analyses. XRF analyses at UAF were conducted on pressed pellets using standard Norrish and Hutton (1969) procedures as described in Potts (1987) and as used by Cameron (2000). Well-calibrated international standards were used as a check of the analyses. The routine was customized for inter-elemental X-ray interferences in rocks of basaltic composition. The UAF-derived XRF analyses for Y are significantly (5-25\%) higher than those reported by ALS Chemex (both analyses are reported in table 4). The UAF Y values are more comparable to the independently derived Y XRF analyses of Amato and others (2003) [run at New Mexico State University] for basalts and gabbros of the same age in the Nome Quadrangle immediately to the west of the Council project area. We conclude that all of the UAF-derived trace-element analyses ( $\mathrm{Nb}, \mathrm{Rb}, \mathrm{Sr}, \mathrm{Y}$, and $\mathrm{Zr}$ ) more closely reflect the true concentration values, and hence recommend the use of the UAF values over those reported by ALS Chemex.

All non-carbonate carbon analyses (table 7) were performed by ALS Chemex. Non-carbonate carbon concentrations were determined by Leco induction furnace pyrolysis following a dilute acid digestion. Analytical methods and detection limits are tabulated in Table 7.

\section{ACKNOWLEDGMENTS}

We would like to thank Laurel E. Burns for reviewing the text and tables in this report. This project is part of the Alaska Airborne Geophysical/Geological Mineral Inventory Program funded by the Alaska State Legislature and managed by State of Alaska, Department of Natural Resources, Division of Geological \& Geophysical Surveys. Partial funding for the geologic mapping and geochemical analyses was also provided through the U.S. Geological Survey STATEMAP Program under award number 06HQAG0024.

\section{REFERENCES CITED}

Amato, J.M., Miller, E.L., Wright, J.E., and McIntosh, W.C., 2003, Dike swarms on Seward Peninsula, Alaska, and their implications for the kinematics of Cretaceous extension in the Bering Strait region: Canadian Journal of Earth Sciences, v. 40, p. 865-886.

Cameron, C.E., 2000, Fault-hosted Au mineralization, Ester Dome, Alaska: Fairbanks, Alaska, M.S. thesis, University of Alaska Fairbanks, 115 p.

Noorish, K., and Hutton, J.T., 1969, An accurate X-ray spectrographic method for the analysis of a wide range of geologic samples: Geochimica et Cosmochimica Acta, v. 33, p. 431-453.

Potts, P.J., 1987, A handbook of silicate rock analysis: Glasgow, Blackie, 622 p. 
Table 1. Location and description of rocks collected for trace-element geochemical analyses in the Solomon Quadrangle, Seward Peninsula, Alaska.

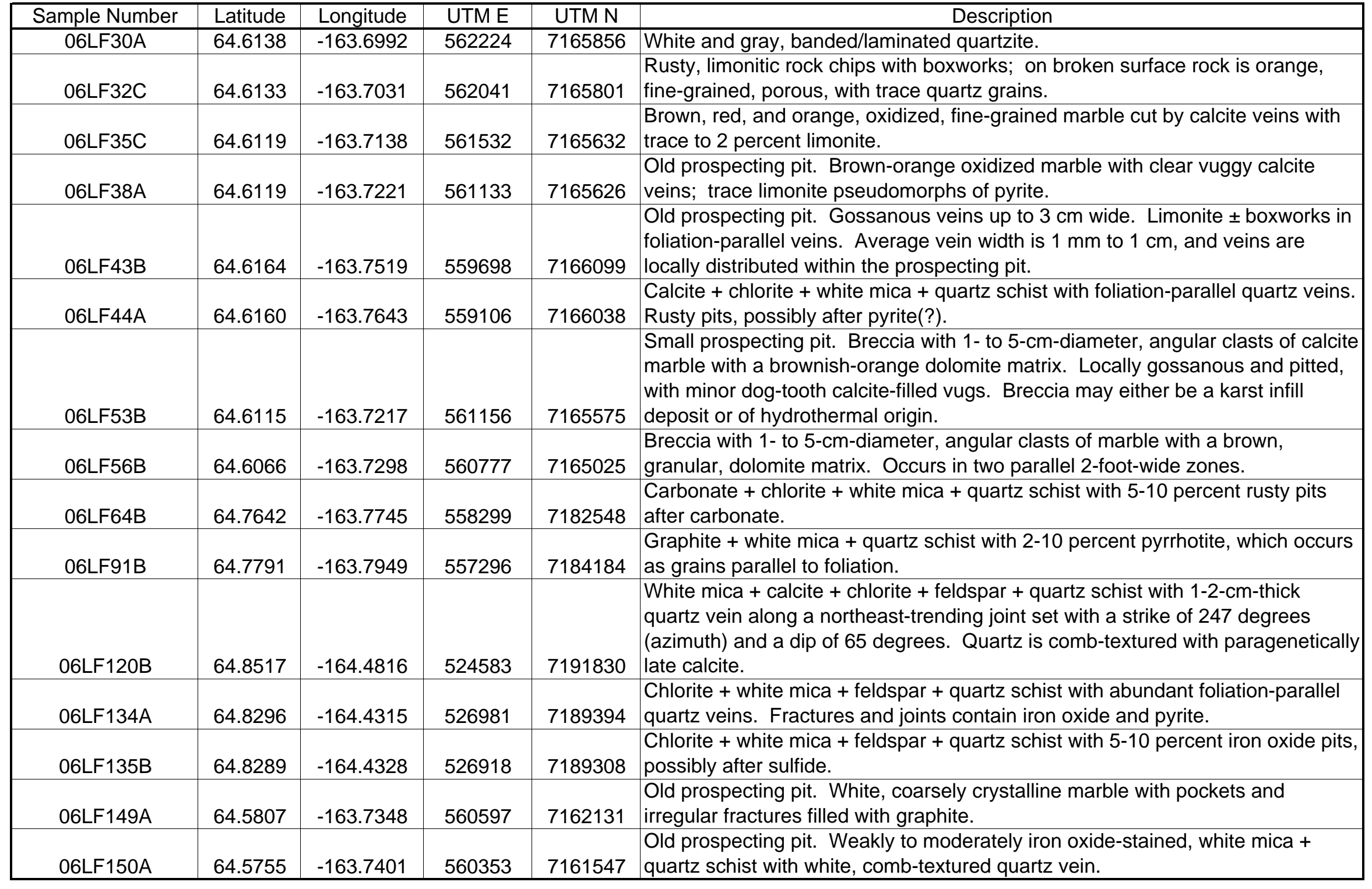


Table 1. (continued).

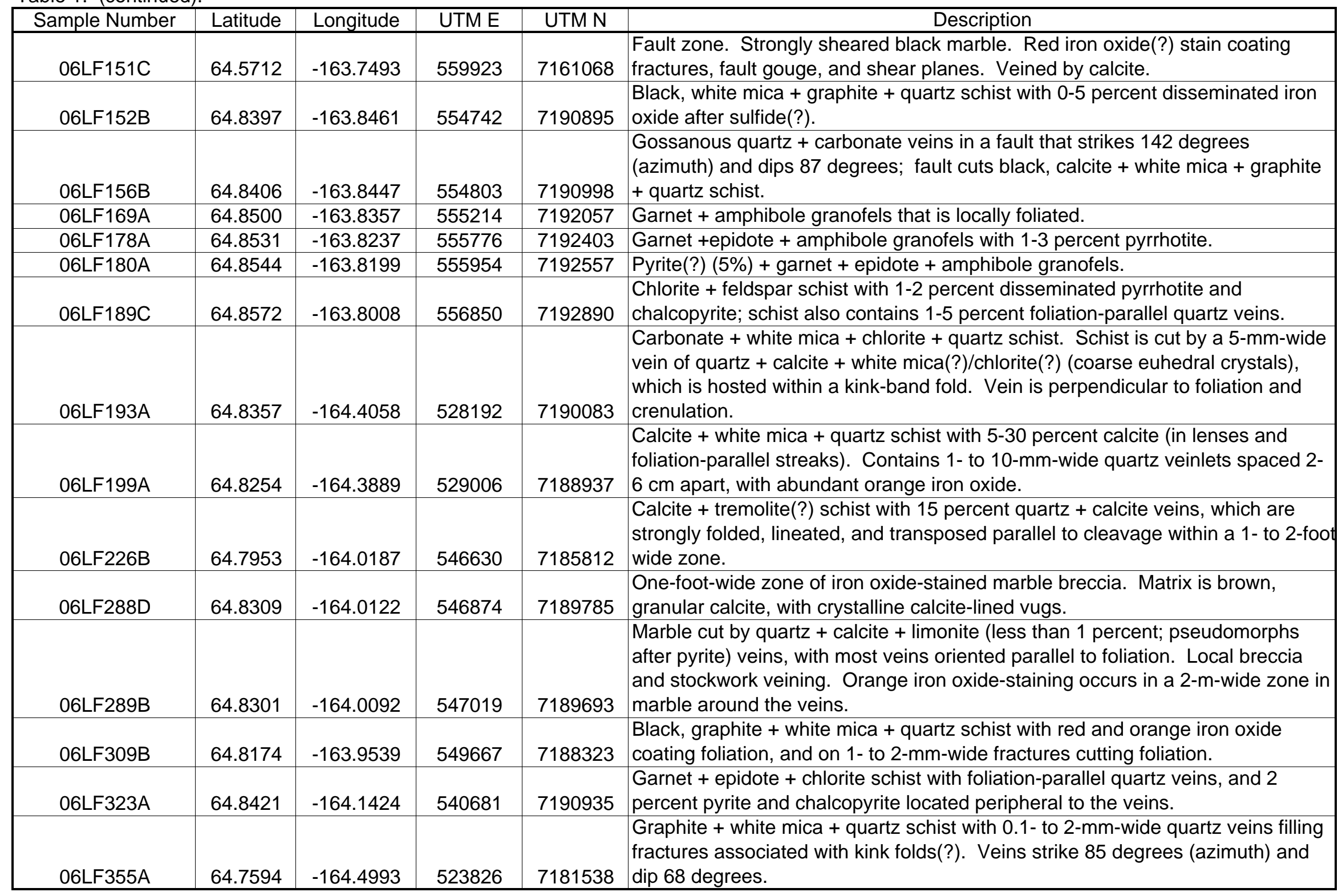


Table 1. (continued).

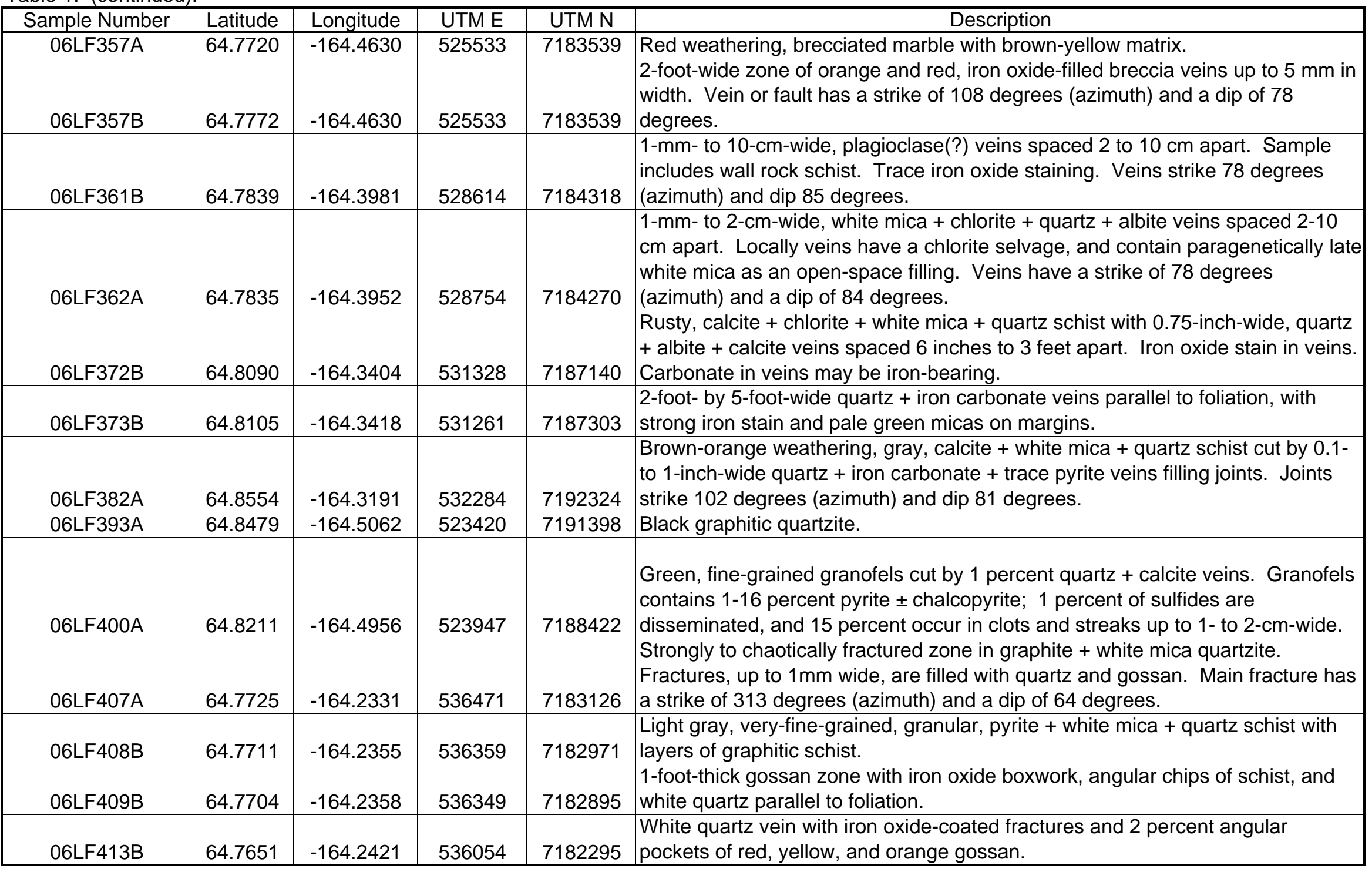


Table 1. (continued).

\begin{tabular}{|c|c|c|c|c|c|}
\hline Sample Number & Latitude & Longitude & UTM E & UTM N & Description \\
\hline 06LF442B & 64.8849 & -164.2139 & 537230 & 7195660 & $\begin{array}{l}\text { Black, very sooty, graphite + quartz schist with fine-grained, alternating layers of } \\
\text { quartz and graphite. }\end{array}$ \\
\hline 06LF449A & 64.8971 & -164.1950 & 538107 & 7197034 & $\begin{array}{l}\text { Orange-tan weathering, greenish-gray, impure dolomitic marble with trace to } 1 \\
\text { percent pyrite, and foliation defined by greenish-yellow mica crystals. Cut by } 10 \\
\text { percent, one-quarter-inch-wide calcite veinlets. }\end{array}$ \\
\hline 06LF457A & 64.8416 & -164.0861 & 543351 & 7190925 & $\begin{array}{l}\text { Tan weathering, white mica + calcite schist with iron oxide along fractures and in } \\
\text { lenses up to } 1 \mathrm{~mm} \text { thick. }\end{array}$ \\
\hline 06LF489A & 64.7923 & -164.0568 & 544824 & 7185447 & $\begin{array}{l}\text { Green, chlorite-spotted, chlorite }+ \text { calcite }+ \text { epidote }+ \text { feldspar schist cut by } \\
\text { calcite-filled, red iron oxide(?)-coated fractures. }\end{array}$ \\
\hline 06LF492A & 64.7947 & -164.0627 & 544539 & 7185706 & $\begin{array}{l}\text { Orange, tan, and gray, fine-grained, foliated marble cut by abundant white } \\
\text { calcite veinlets. Marble contains iron-stained, sooty graphite-filled fractures, } \\
\text { voids, and matrix. }\end{array}$ \\
\hline 06LF499B & 64.7583 & -164.4693 & 525255 & 7181426 & $\begin{array}{l}\text { Tan weathering, impure schistose marble, which occurs in discontinuous layers } \\
\text { or in the cores of isoclinal folds(?). Pyrite and chalcopyrite occur in } 0.05 \text {-inch- } \\
\text { thick siliceous bands, and as fracture coatings in brecciated impure marble over } \\
\text { a } 15 \text {-foot by } 15 \text {-foot area. The mineralized zone is about } 2 \text { to } 4 \text { inches thick. }\end{array}$ \\
\hline 06LF501B & 64.7581 & -164.4714 & 525154 & 7181408 & White, granular, fine-grained, silicified marble(?) with relict banding/foliation. \\
\hline 06LF501C & 64.7581 & -164.4714 & 525154 & 7181408 & $\begin{array}{l}\text { Brown weathering, oxidized rock. Light brown, fine-grained rock with orange, } \\
\text { limonite-filled pockets between } 0.1 \text { and } 0.3 \text { inches in diameter. }\end{array}$ \\
\hline
\end{tabular}


Table 1. (continued).

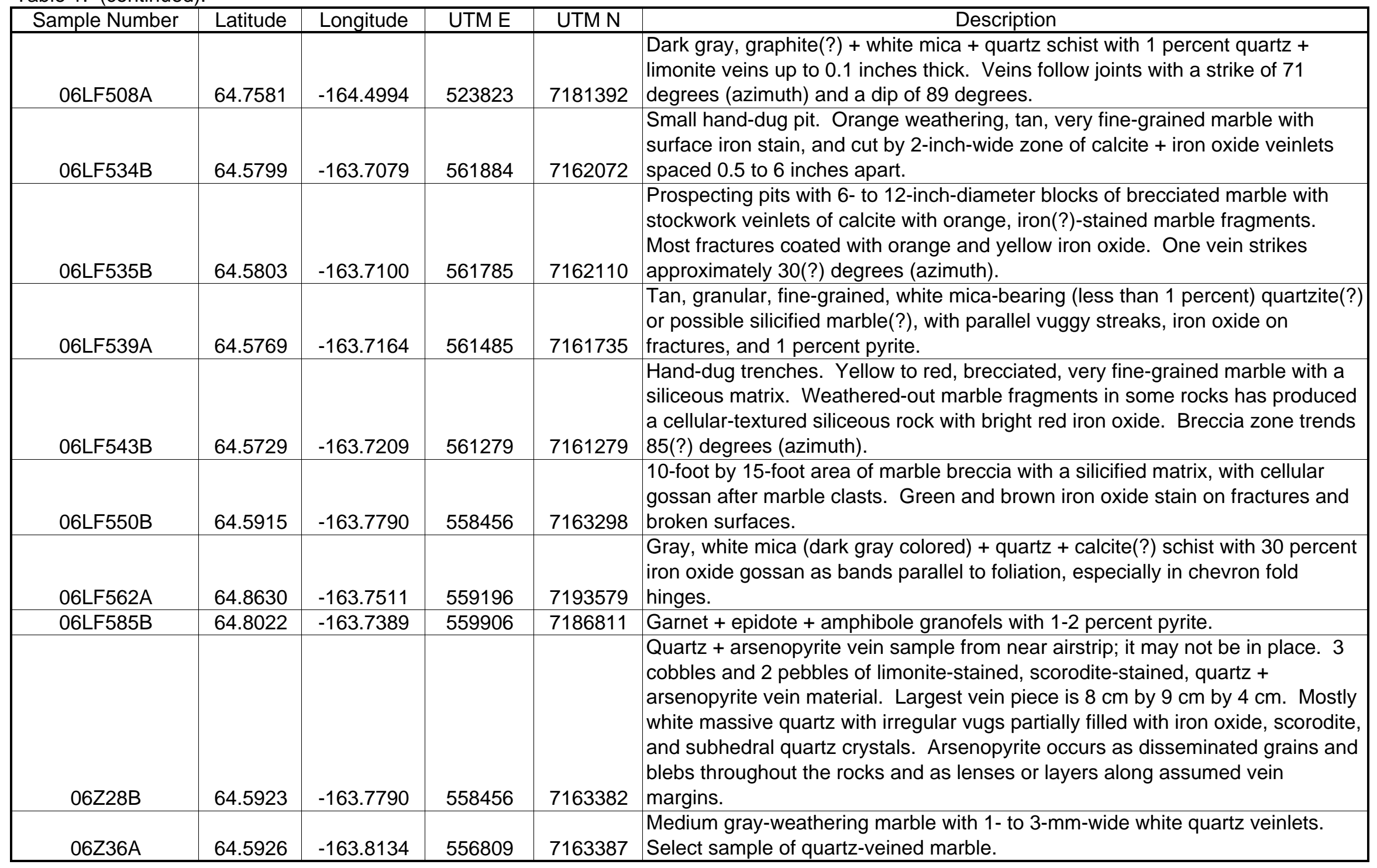


Table 1. (continued).

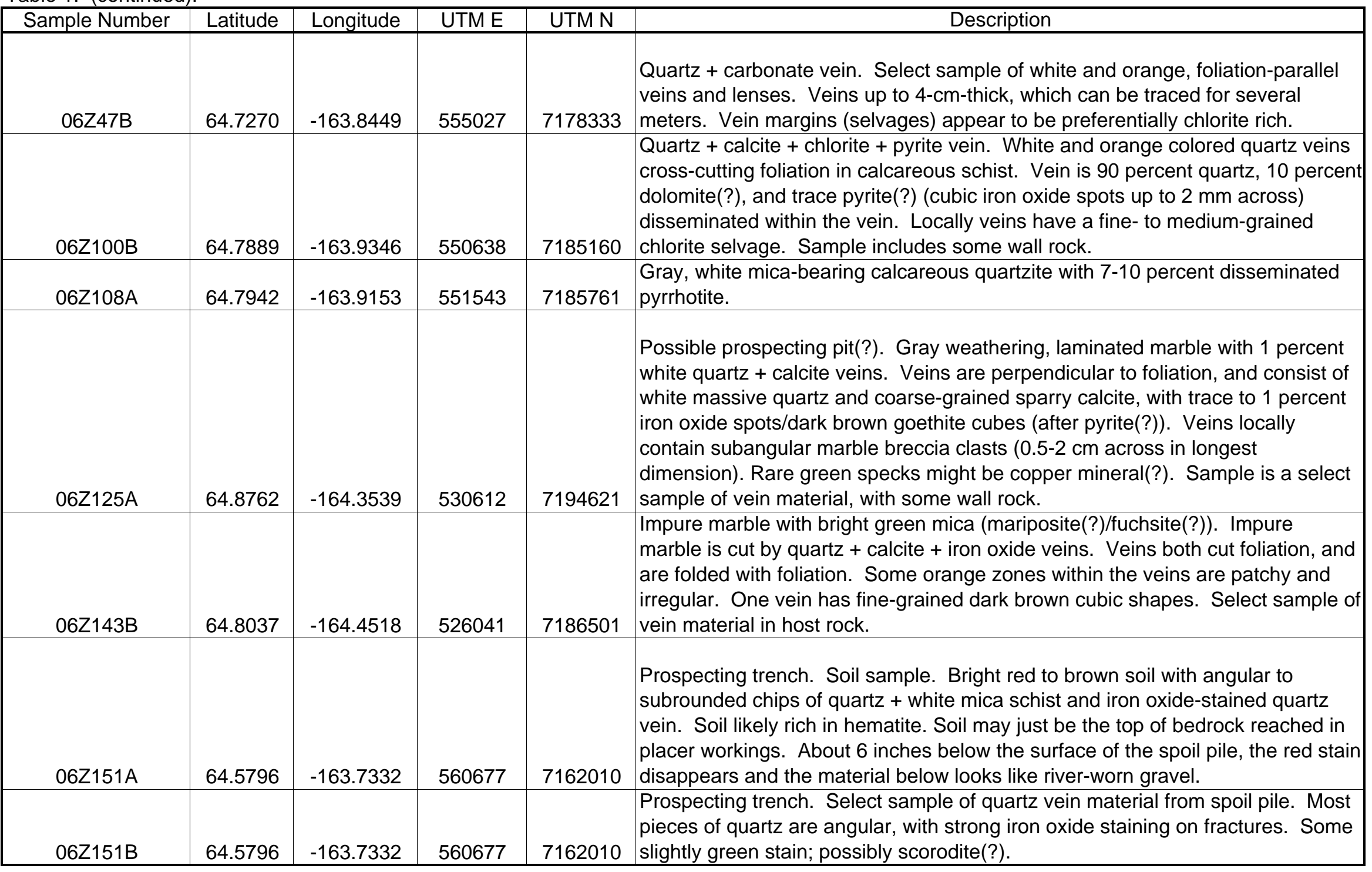


Table 1. (continued).

\begin{tabular}{|c|c|c|c|c|c|}
\hline Sample Number & Latitude & Longitude & UTM E & UTM N & Description \\
\hline 06Z156A & 64.5677 & -163.6965 & 562461 & 7160727 & $\begin{array}{l}\text { Swede lode. Observed } 1 \text { shaft and } 3 \text { prospecting pits within } 50 \text {-foot-diameter } \\
\text { area. Collected sample from ore pile approximately } 25 \text { feet from cliff. Calcite }+ \\
\text { cinnabar breccia. Pink, light brown, and white breccia consisting of brown } \\
\text { angular clasts of marble, from } 1 \mathrm{~mm} \text { to } 4 \mathrm{~cm} \text { in diameter, cemented by a white } \\
\text { calcite matrix. Red cinnabar crusts and irregular grains make up approximately } \\
5 \text { percent of the rock. Weathered surfaces coated with black, mm-diameter, } \\
\text { semi-equant grains (oxidized sulfides(?)); limonite is also present in moderate } \\
\text { amounts. }\end{array}$ \\
\hline 06Z161A & 64.8338 & -163.9668 & 549025 & 7190137 & $\begin{array}{l}\text { Dark gray, tightly crenulated, quartz + white mica schist with } 2-3 \text { percent, fine- } \\
\text { grained (up to } 1 \mathrm{~mm} \text { diameter) disseminated grains and blebs of brass-colored } \\
\text { sulfide (likely pyrrhotite). Outcrop has } 1-2 \text { percent, foliation-parallel quartz veins } \\
\text { Sample taken of pyrrhotite-bearing schist collected over an } 8 \text {-foot section along } \\
\text { creek. }\end{array}$ \\
\hline 06Z179A & 64.8393 & -164.3944 & 528730 & 7190492 & $\begin{array}{l}\text { Light brown quartzite with minor white mica, chlorite, and calcite, along with 3-4 } \\
\text { percent disseminated pyrite up to } 1 \mathrm{~mm} \text { in diameter. Weak iron oxide stain on } \\
\text { weathered surfaces, and moderate iron oxide on fractures. Some pods of light } \\
\text { brown/orange iron oxide in open spaces. Grab sample over 15-foot-diameter } \\
\text { area. }\end{array}$ \\
\hline 06Z196B & 64.7685 & -164.1353 & 541130 & 7182745 & $\begin{array}{l}\text { Quartz + epidote + chlorite + feldspar(?) veins. White cobbles and boulders up } \\
\text { to } 20 \mathrm{~cm} \text { in diameter. Many veins of white bull quartz, but some of the massive } \\
\text { quartz veins also have granular texture like pegmatite veins. Several veins have } \\
\text { malachite spots. Sample collected over } 30 \text {-foot-diameter area. Most } \\
\text { vein/pegmatites have coarse-grained chlorite and iron oxide. At least one vein } \\
\text { had epidote crystals up to } 5 \mathrm{~cm} \text { long by } 1 \mathrm{~cm} \text { wide, along with chlorite }+ \\
\text { biotite(?) and feldspar(?). Trace amounts of non-magnetic, black unknown } \\
\text { mineral, and trace, bright-red hematite(?) stain. }\end{array}$ \\
\hline
\end{tabular}


Table 1. (continued).

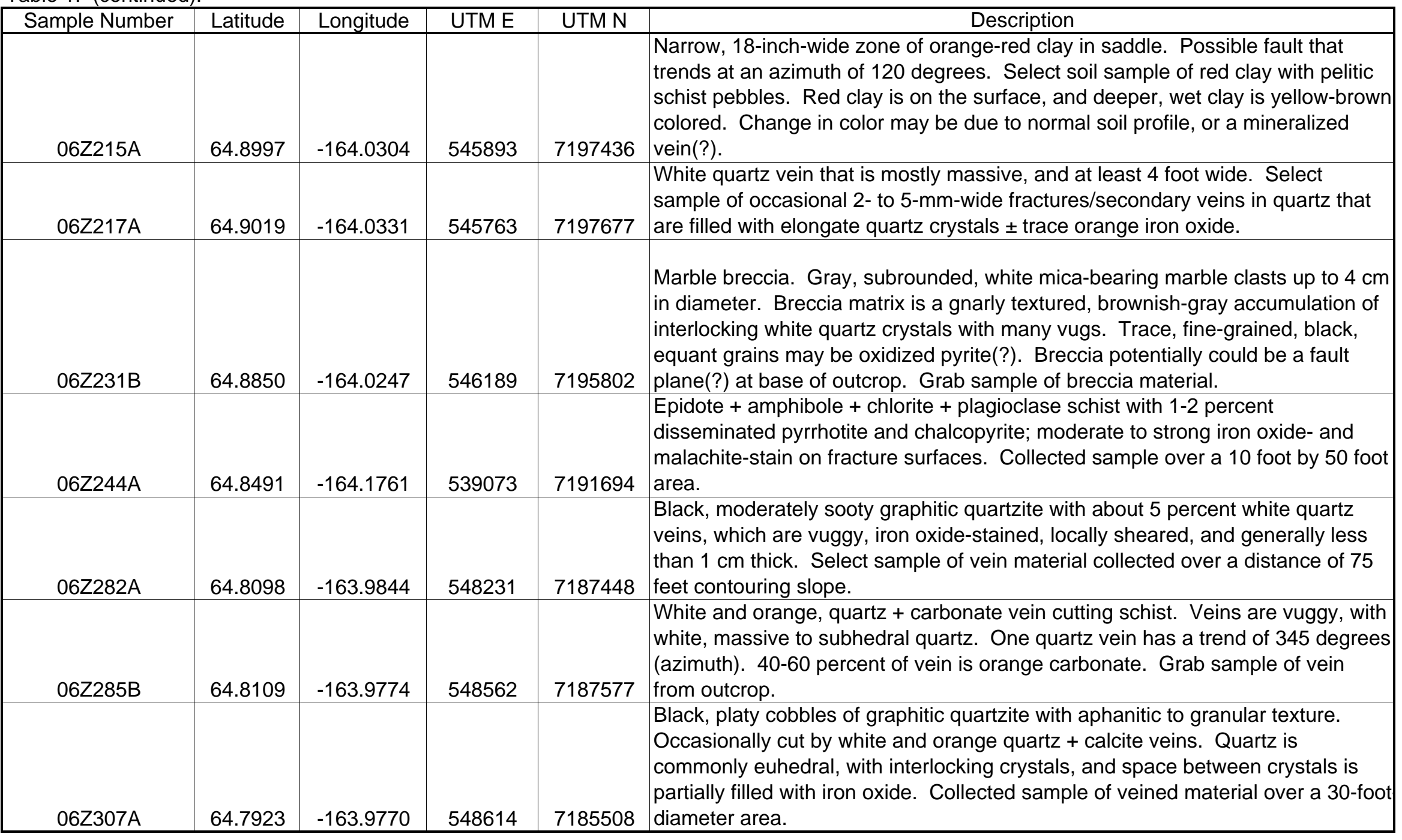


Table 1. (continued).

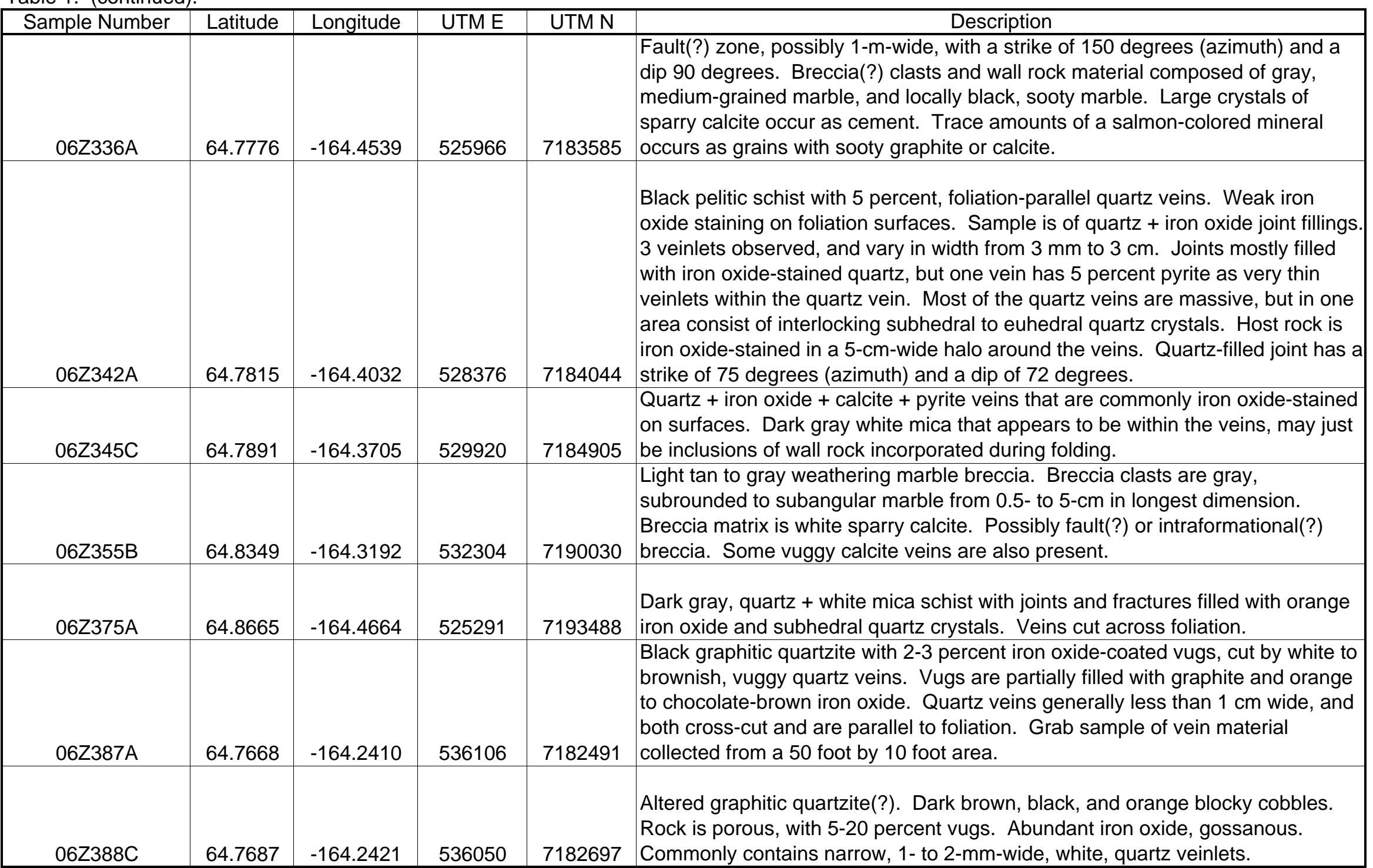


Table 1. (continued).

\begin{tabular}{|c|c|c|c|c|c|}
\hline Sample Number & Latitude & Longitude & UTM E & UTM N & Description \\
\hline 06Z404A & 64.7799 & -164.2730 & 534564 & 7183927 & $\begin{array}{l}\text { Dark gray, granular, fine-grained, white mica-bearing marble with } 3-4 \text { percent } \\
\text { disseminated pyrite up to } 1 \mathrm{~mm} \text { across, but generally less than } 0.5 \mathrm{~mm} \text {. }\end{array}$ \\
\hline 06Z412B & 64.7614 & -164.2408 & 536123 & 7181891 & $\begin{array}{l}\text { Dark brown to orange gossan. Abundant iron oxide. Some pieces are } \\
\text { brecciated, with graphitic quartzite clasts surrounded by brown iron oxide. Grab } \\
\text { sample taken over 15-square-foot area. }\end{array}$ \\
\hline 06Z420A & 64.9263 & -164.1390 & 540715 & 7200324 & $\begin{array}{l}\text { Dark gray, calcite + white mica + quartz schist with } 5 \text { percent white quartz } \\
\text { veinlets. Veins are both parallel to and cross foliation. } 1 \text { percent fine-grained, } \\
\text { disseminated pyrite cubes and irregular clots. Schist also cut by quartz + calcite } \\
\text { veinlets, which cross foliation. }\end{array}$ \\
\hline 06Z430A & 64.9129 & -164.1822 & 538690 & 7198808 & $\begin{array}{l}\text { Dark gray and white, calcite }+ \text { white mica }+ \text { quartz schist with } 1 \text { percent, fine- } \\
\text { grained, disseminated pyrite, and } 7 \text { percent, white, foliation-parallel quartz }+ \\
\text { calcite }+ \text { iron oxide veins. Veins are } 1-\text { to } 2-c m-t h i c k, \text { and locally folded and } \\
\text { structurally thickened. }\end{array}$ \\
\hline 06Z489B & 64.8581 & -164.4227 & 527369 & 7192568 & $\begin{array}{l}\text { White, pale brown, and gray marble breccia. Matrix composed of white quartz } \\
\text { with trace disseminated pyrite and minor iron oxide stain. Marble breccia clasts } \\
\text { are dark gray to bleached(?) light orange, subrounded, and up to } 8 \mathrm{~cm} \text { in length. } \\
\text { Breccia also contains schist clasts, or schist pieces are part of wall rock. }\end{array}$ \\
\hline 06Z490A & 64.8573 & -164.4175 & 527615 & 7192487 & ght to dark gray, silicified marble with patches of orange and red iron oxide. \\
\hline
\end{tabular}


Table 1. (continued).

\begin{tabular}{|c|c|c|c|c|c|}
\hline Sample Number & Latitude & Longitude & UTM E & UTM N & Description \\
\hline 06Z492A & 64.5710 & -163.7467 & 560045 & 7161048 & $\begin{array}{l}\text { Idaho lode prospect. Quartz + calcite + white mica + arsenopyrite vein, which } \\
\text { varies from } 10 \mathrm{~cm} \text { to } 95 \mathrm{~cm} \text { in thickness. In places the vein is entirely massive } \\
\text { sulfide, and in other areas it consists of about } 10 \text { percent sulfide (arsenopyrite } \\
\text { and pyrite cubes) and a mixture of white quartz, white to orange carbonate, and } \\
\text { coarse-grained white mica. Vein is folded, and cuts foliation in marble; on cliff } \\
\text { above, quartz + arsenopyrite vein cuts through schist. Vein is located near the } \\
\text { contact between schist and marble. Sample from arsenopyrite-rich portion of } \\
\text { vein. }\end{array}$ \\
\hline 06Z492D & 64.5710 & -163.7467 & 560045 & 7161048 & $\begin{array}{l}\text { Idaho lode prospect. Quartz + calcite + white mica + arsenopyrite vein, which } \\
\text { varies from } 10 \mathrm{~cm} \text { to } 95 \mathrm{~cm} \text { in thickness. In places the vein is entirely massive } \\
\text { sulfide, and in other areas it consists of about } 10 \text { percent sulfide (arsenopyrite } \\
\text { and pyrite cubes) and a mixture of white quartz, white to orange carbonate, and } \\
\text { coarse-grained white mica. Vein is folded, and cuts foliation in marble; on cliff } \\
\text { above, quartz + arsenopyrite vein cuts through schist. Vein is located near the } \\
\text { contact between schist and marble. Sample from quartz- and calcite-rich } \\
\text { portion of vein. }\end{array}$ \\
\hline 06Z523B & 64.7510 & -163.9710 & 548974 & 7180911 & $\begin{array}{l}\text { Black graphitic quartzite with abundant white quartz veinlets cutting across } \\
\text { foliation. Some open spaces in veins filled with orange and red iron oxide stain. }\end{array}$ \\
\hline 06MBW48A & 64.6815 & -164.4893 & 524372 & 7172868 & $\begin{array}{l}\text { High grade sample from dump pile at West Creek prospect. Massive, white, } \\
\text { heavily iron-stained, } 3(?) \text {-meter-wide quartz vein on hillside. Quartz vein cut by } \\
\text { later fractures, with open space } \pm \text { filled with euhedral quartz crystals. Adit below } \\
\text { quartz vein has a dump pile just down stream. Took sample of dump material, } \\
\text { which contains quartz + massive arsenopyrite + cubic pyrite + white kaolinite } \\
\text { (confirmed by X-ray diffraction) + carbonate. }\end{array}$ \\
\hline 06MBW50A & 64.7431 & -164.0154 & 546878 & 7179997 & $\begin{array}{l}\text { Quartz + plagioclase vein in schist within a low-angle fault. Foliation in schist } \\
\text { above the vein is highly folded and sheared, suggesting faulting occurred within } \\
\text { this zone. }\end{array}$ \\
\hline 06MBW73A & 64.6252 & -163.8688 & 554089 & 7166976 & $\begin{array}{l}\text { Black, foliated, graphitic quartzite cut perpendicular to foliation by } 3-\text { to } 6-\mathrm{mm}- \\
\text { wide quartz + limonite veins. Quartz is crystalline and growing perpendicular to } \\
\text { vein walls. Most limonite likely was calcite. Locally limonite is clear and glassy } \\
\text { (bottle-glass limonite), suggesting sulfides may have been present. Couple of } \\
\text { pieces of quartzite are brecciated, with limonite cement. }\end{array}$ \\
\hline
\end{tabular}


Table 1. (continued).

\begin{tabular}{|c|c|c|c|c|c|}
\hline Sample Number & Latitude & Longitude & UTM E & UTM N & Description \\
\hline 06MBW81A & 64.6156 & -163.6946 & 562442 & 7166061 & $\begin{array}{l}\text { Chlorite + white mica + biotite(?) + quartz schist with foliation-parallel, folded } \\
\text { quartz + limonite veins. Sampled 2-inch-wide folded quartz vein with about } 5 \\
\text { percent patches of limonite (after carbonate(?)). }\end{array}$ \\
\hline 06MBW82C & 64.6124 & -163.6949 & 562436 & 7165707 & $\begin{array}{l}\text { Contact between schist to the east and marble to the west. Contact zone } \\
\text { contains gray and white striped, granular, silicified marble with relict foliation } \\
\text { visible. }\end{array}$ \\
\hline 06MBW83C & 64.6126 & -163.6955 & 562406 & 7165726 & $\begin{array}{l}\text { Gray, partially to completely silicified, } \pm \text { brecciated, color-banded marble that } \\
\text { contains disseminated pyrite and possibly chalcopyrite. Local thin coatings of } \\
\text { malachite and azurite on surfaces of rocks. Locally veined by limonite + calcite } \\
+ \text { cubic limonite pseudomorphs after pyrite. }\end{array}$ \\
\hline 06MBW86A & 64.6098 & -163.6980 & 562292 & 7165410 & $\begin{array}{l}\text { Prospecting pit. Gray, partially to completely silicified calcite marble cut by } \\
\text { quartz veins within foliation planes and quartz veins that cross foliation. Silicified } \\
\text { and quartz-veined areas contain disseminated chalcopyrite, variably weathered } \\
\text { to limonite, azurite, and malachite. Some pyrite is also present. Quartz veins } \\
\text { are white, massive, and granular. Trend of surface vein float has an azimuth of } \\
\text { about } 0 \text { degrees; the veined zone varies from } 0 \text { to } 20 \text { feet wide, and extends for } \\
\text { about } 150 \text { feet in a north-south direction. Some veins are folded, and measured } \\
\text { fold axes have a strike of about } 175 \text { degrees (azimuth) and a dip of } 65 \text { degrees. }\end{array}$ \\
\hline 06MBW90B & 64.6055 & -163.6982 & 562293 & 7164939 & $\begin{array}{l}\text { Silicified marble with limonite (after sulfides(?)) in patches and stringers } \\
\text { throughout the rock. }\end{array}$ \\
\hline 06MBW90C & 64.6055 & -163.6982 & 562293 & 7164939 & $\begin{array}{l}\text { Dark gray, graphitic breccia composed of 2-mm-diameter, angular marble host- } \\
\text { rock clasts and cemented by a graphite-rich matrix. Probable fault zone } \\
\text { material. }\end{array}$ \\
\hline 06MBW92A & 64.6048 & -163.7023 & 562096 & 7164853 & $\begin{array}{l}\text { 20-foot-wide zone of silicified marble that has an azimuth of } 20 \text { degrees. } \\
\text { Silicified marble is composed of white, granular quartz with streaks of gray } \\
\text { (graphite(?)). }\end{array}$ \\
\hline 06MBW107A & 64.6215 & -163.6985 & 562239 & 7166714 & $\begin{array}{l}\text { White weathering, granular, silicified marble with white and light gray color- } \\
\text { banding along relict foliation planes. Silicified marble contains } 1-3 \text { percent } \\
\text { disseminated grains of chalcopyrite and pyrite. Sulfides weathering to limonite } \\
\text { and malachite and lesser azurite. }\end{array}$ \\
\hline 06MBW161A & 64.7835 & -164.0100 & 547064 & 7184498 & $\begin{array}{l}\text { Quartz veins with sparse iron oxide pseudomorphs after iron-bearing sulfide, } \\
\text { with minor quartz + white mica schist wall rock included in sample. }\end{array}$ \\
\hline
\end{tabular}


Table 1. (continued).

\begin{tabular}{|c|c|c|c|c|c|}
\hline Sample Number & Latitude & Longitude & UTM E & UTM N & Description \\
\hline 06MBW163A & 64.7842 & -164.0103 & 547048 & 7184574 & $\begin{array}{l}\text { Quartz + calcite vein in marble. Quartz vein is massive, white, brittle-fractured, } \\
\text { and strikes approximately } 150 \text { degrees (azimuth). }\end{array}$ \\
\hline 06MBW168A & 64.7880 & -164.0061 & 547242 & 7185006 & $\begin{array}{l}\text { Fissile calcite marble with white quartz vein sub-parallel to foliation. The marble } \\
\text { adjacent to the quartz vein has crystals of pale green tremolite(?) (up to } 1 \mathrm{~cm} \text { in } \\
\text { diameter and up to } 3 \mathrm{~cm} \text { in length), and coarsely crystalline white mica up to } 3 \\
\text { mm in diameter. Sampled the calcite + tremolite(?) + white mica + quartz- } \\
\text { altered wall rock and vein material. Reaction zone is } 2-3 \text { inches wide. Quartz } \\
\text { vein has a strike of } 140 \text { degrees (azimuth). }\end{array}$ \\
\hline 06MBW179A & 64.8294 & -163.8025 & 556832 & 7189784 & Quartz + chlorite vein in schist. \\
\hline 06MBW189A & 64.7842 & -163.7751 & 558226 & 7184778 & $\begin{array}{l}\text { Calcium-carbonate travertine deposited out of the water coming out of a spring } \\
\text { on hillside above tiny creek. Surface weathering or oxidation colors of travertine } \\
\text { include black, tan, orange, light brown, and dark rusty orange-brown. Freshly } \\
\text { broken surfaces are usually pale brown. Travertine deposits are usually } \\
\text { layered, with horizons of vugs; deposits sink up to } 2.5 \text { feet when stepped upon. } \\
\text { Calcium carbonate also forms botryoidal-textured deposits, and "pond and } \\
\text { terrace" deposits; empty ponds commonly contain round, pea-sized pisoliths. } \\
\text { Inclusions of leaf and twig debris are common, and the deposits have formed a } \\
\text { natural kill zone in the vegetation around the spring. The calcium carbonate has } \\
\text { been deposited over an area about } 200 \text { feet in diameter. }\end{array}$ \\
\hline 06MBW232A & 64.8366 & -164.4195 & 527544 & 7190172 & $\begin{array}{l}\text { Lumpy, fractured quartz + carbonate + chlorite + white mica(?) vein in tundra } \\
\text { with schist host rock. }\end{array}$ \\
\hline 06MBW262A & 64.8145 & -164.2319 & 536475 & 7187811 & $\begin{array}{l}\text { Black, sooty, very fine-grained, foliated, isoclinally folded, graphitic schist and } \\
\text { quartzite. Locally lightly iron oxide-coated on fracture surfaces. Rocks contain } \\
\text { varying proportions of graphite (1-8 percent), iron sulfide (1-2 percent pyrite(?) } \\
\text { weathering to limonite), white mica (trace to } 20 \text { percent in schist), and primarily } \\
\text { quartz. Locally cut by quartz } \pm \text { iron sulfide veins. Veins cut foliation, and in most } \\
\text { cases appear to be tension-related since quartz crystals grow off of vein } \\
\text { margins into the vein center. Sampled select pieces with } 3-5 \text { percent veins. }\end{array}$ \\
\hline
\end{tabular}


Table 1. (continued).

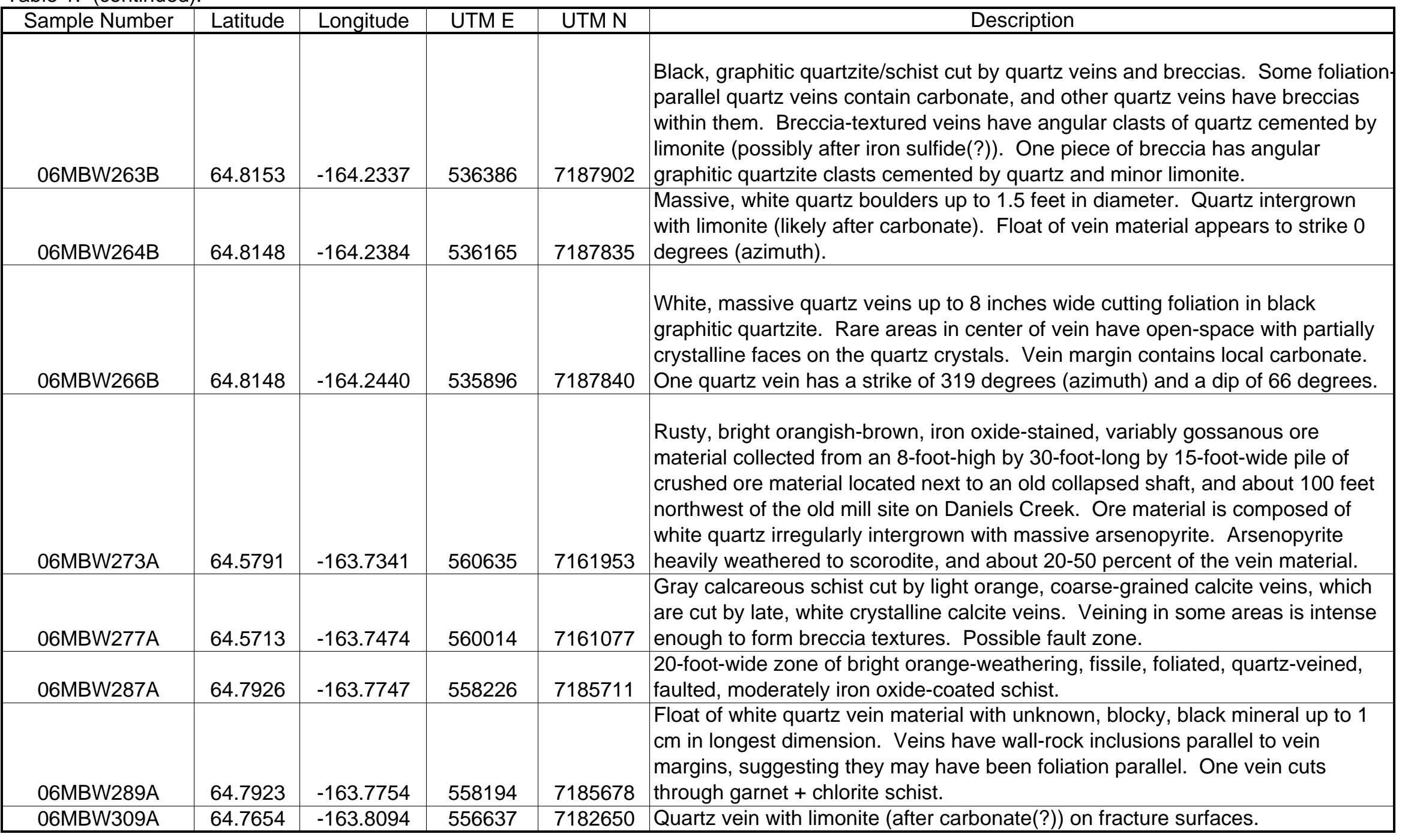


Table 1. (continued).

\begin{tabular}{|c|c|c|c|c|c|}
\hline Sample Number & Latitude & Longitude & UTM E & UTM N & Description \\
\hline 06MBW330A & 64.8269 & -164.3062 & 532928 & 7189151 & $\begin{array}{l}\text { Fault zone that is at least } 40 \text { feet wide and has an approximate strike of } 305 \\
\text { degrees (azimuth) and an estimated dip of } 65 \text { degrees. Brownish-orange } \\
\text { weathering, bright brownish-orange colored, blocky, brecciated, calcite marble. } \\
\text { Breccia clasts composed of marble and large, gray, secondary crystals of calcite } \\
\text { up to } 1 \mathrm{~cm} \text { in length. All clasts are cemented and cut by late veins of calcite. }\end{array}$ \\
\hline 06MBW336A & 64.7815 & -164.1695 & 539485 & 7184168 & $\begin{array}{l}\text { Large boulders of vein material up to } 3.5 \text { feet in diameter. Primarily white, } \\
\text { massive, fractured, quartz ( } 85 \text { percent), intergrown with lesser (10 percent), tan, } \\
\text { euhedral feldspar crystals (up to } 2.5 \mathrm{~cm} \text { long; averaging less than } 1 \mathrm{~cm} \text { in } \\
\text { length), and dark green chlorite (5 percent). Quartz boulder float trends roughly } \\
270(?) \text { degrees (azimuth). }\end{array}$ \\
\hline 06MBW403A & 64.8159 & -164.2108 & 537471 & 7187976 & $\begin{array}{l}\text { Black, very fine-grained, planar-foliated, graphitic quartzite. Lightly coated with } \\
\text { iron oxide on some fractures. Other joint surfaces contain quartz + limonite } \\
\text { (after iron sulfides(?)) veins up to } 1 \mathrm{~cm} \text { wide. Veins are probably extensional } \\
\text { since the quartz crystals grow off the walls of the veins towards the center. } \\
\text { Veins form en echelon tensional joint sets. One quartz + limonite vein has a } \\
\text { strike of } 323 \text { degrees (azimuth) and a dip of } 40 \text { degrees. }\end{array}$ \\
\hline 06MBW438A & 64.8308 & -164.1886 & 538504 & 7189653 & $\begin{array}{l}\text { 10-foot-wide brecciated fault zone in marble, which has a strike of about } 320 \\
\text { degrees (azimuth) and may dip to the northeast. Gray calcite marble is } \\
\text { brecciated, with angular clasts of marble cemented by bright orange-weathering } \\
\text { crystalline carbonate. Some brecciation didn't involve clast rotation - it is just a } \\
\text { crackle breccia. }\end{array}$ \\
\hline 06MBW454A & 64.8385 & -164.1992 & 537991 & 7190498 & $\begin{array}{l}\text { Black graphitic quartzite cut by irregular quartz + limonite veins and breccias. } \\
\text { Breccia clasts of quartzite average } 1-2 \text { inches in diameter, and are cemented by } \\
\text { iron oxide. }\end{array}$ \\
\hline 06MBW465A & 64.7903 & -164.1816 & 538895 & 7185144 & $\begin{array}{l}\text { Gray weathering calcite marble with white and gray color banding sub-parallel to } \\
\text { foliation. Locally silicified marble layers are interlayered with pure marble. } \\
\text { Weathers orange and tan where silicified. }\end{array}$ \\
\hline 06MBW472A & 64.7928 & -164.1815 & 538896 & 7185419 & $\begin{array}{l}\text { Tan weathering, planar-foliated marble with approximately } 20-25 \text { volume } \\
\text { percent quartz veins parallel to foliation. Either early veins, or later } \\
\text { replacements of marble (less likely). Sampled more siliceous layer. }\end{array}$ \\
\hline
\end{tabular}


Table 1. (continued).

\begin{tabular}{|c|c|c|c|c|c|}
\hline Sample Number & Latitude & Longitude & UTM E & UTM N & Description \\
\hline 06MBW484A & 64.8020 & -164.1741 & 539237 & 7186450 & $\begin{array}{l}\text { Black graphitic quartzite cut across foliation by tensional quartz + limonite + } \\
\text { pyrite veins up to } 4 \mathrm{~cm} \text { wide. Quartz crystals grow towards center of vein, both } \\
\text { perpendicular to, and at an angle to vein walls. Outcrop lightly iron oxide } \\
\text { stained. Quartz + limonite + pyrite vein has a strike of } 302 \text { degrees (azimuth) } \\
\text { and a dip of } 70 \text { degrees. }\end{array}$ \\
\hline 06MBW565A & 64.8167 & -164.1669 & 539554 & 7188094 & $\begin{array}{l}\text { Medium and dark gray, finely color-banded, medium-grained, calcite marble, } \\
\text { with possible minor graphite. Marble contains foliation sub-parallel, and foliation- } \\
\text { cutting quartz + calcite veins up to } 6 \mathrm{~cm} \text { wide. Quartz and calcite are } \\
\text { intergrown. Main vein occurs where the foliation is folded, and may occur within } \\
\text { a fault with minor displacement. Vein/fault has a strike of } 123 \text { degrees (azimuth) } \\
\text { and a dip of } 41 \text { degrees. }\end{array}$ \\
\hline 06MBW568A & 64.8163 & -164.1659 & 539604 & 7188052 & $\begin{array}{l}\text { Heavily iron oxide-coated outcrop of black graphitic quartzite with disseminated } \\
\text { pyrrhotite } \pm \text { pyrite(?). Preferentially sampled rock with highest magnetic } \\
\text { susceptibility and highest visible iron sulfide content ( } 3-5 \text { percent). }\end{array}$ \\
\hline 06MBW572A & 64.8197 & -164.1667 & 539563 & 7188422 & $\begin{array}{l}\text { Black graphitic schist and quartzite cut by tensional veins up to } 3 \mathrm{~cm} \text { wide. } \\
\text { Quartz crystals grow off vein wall into center, and late open space is filled with } \\
\text { limonite. Some veins are parallel to axial planes, and axial-planar cleavage, of } \\
\text { small crenulation folds; these veins strike } 332 \text { degrees (azimuth) and dip } 64 \\
\text { degrees. Sample taken from this vein. Other veins occur along joints; one vein } \\
\text { has a strike of } 134 \text { degrees (azimuth) and a dip of } 85 \text { degrees; another vein has } \\
\text { a strike of } 286 \text { degrees (azimuth) and a dip of } 90 \text { degrees. }\end{array}$ \\
\hline 06MBW573A & 64.8194 & -164.1670 & 539549 & 7188399 & $\begin{array}{l}\text { Outcrop of black graphitic quartzite cut by a low-angle fault. Quartzite above the } \\
\text { fault is planar foliated and relatively undisturbed. Below the fault plane, the } \\
\text { quartzite is highly folded, and contains hundreds of white, massive, randomly } \\
\text { oriented, quartz-filled fractures. Sampled intensely quartz-veined quartzite } \\
\text { below the fault. }\end{array}$ \\
\hline 06MBW589A & 64.7674 & -164.4651 & 525445 & 7182442 & $\begin{array}{l}\text { Gray and white, color-banded marble cut by veins of calcite } \pm \text { iron oxide } \pm \text { red } \\
\text { earthy hematite. Veins anastomose throughout rock in a slightly planar } \\
\text { orientation. Veins pinch and swell, and range from } 0-10 \mathrm{~cm} \text { wide. One calcite } \pm \\
\text { iron oxide vein strikes } 51 \text { degrees (azimuth) and dips } 71 \text { degrees. Another } \\
\text { calcite + hematite vein strikes } 140 \text { degrees (azimuth) and dips } 83 \text { degrees. }\end{array}$ \\
\hline
\end{tabular}


Table 1. (continued).

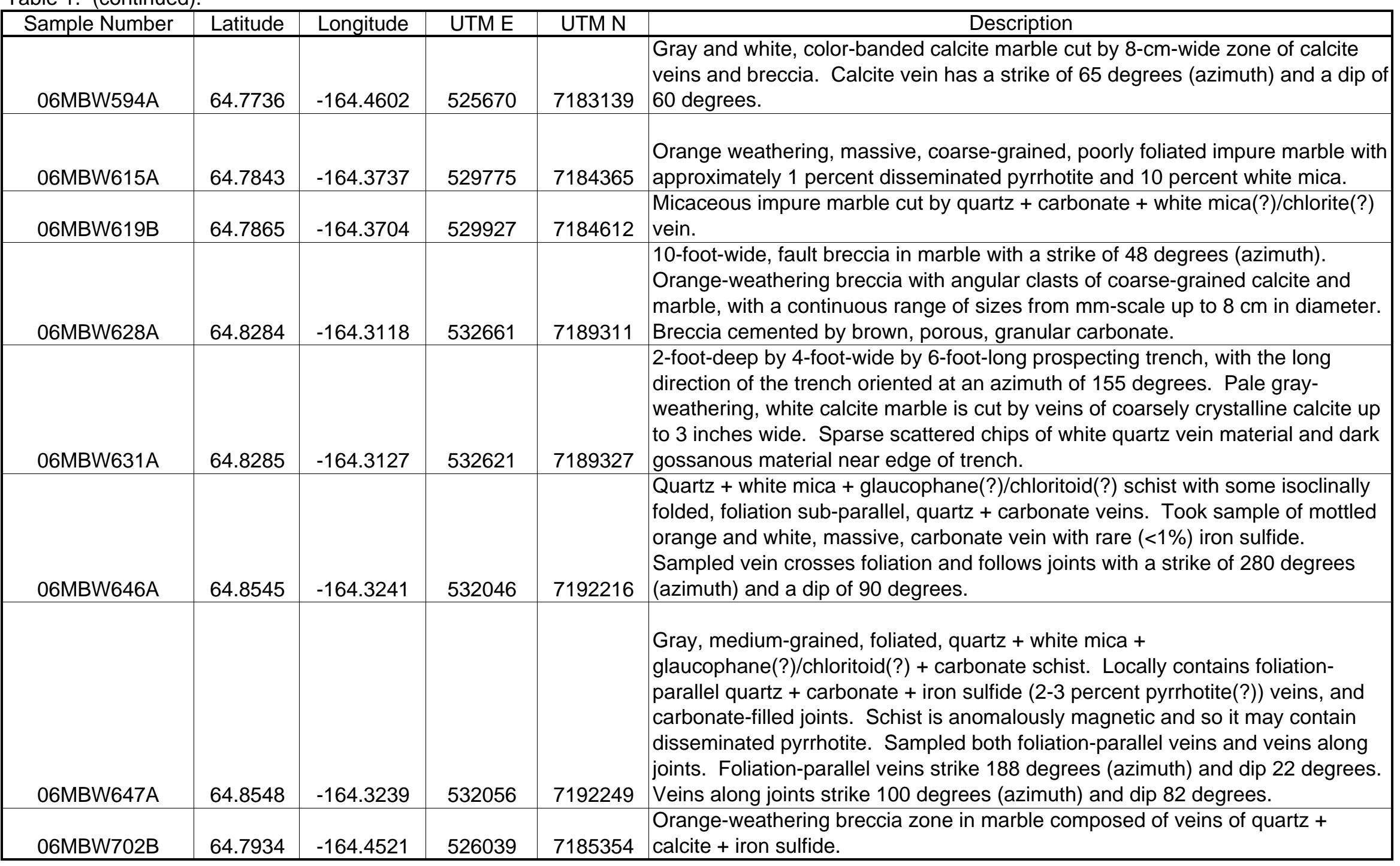


Table 1. (continued).

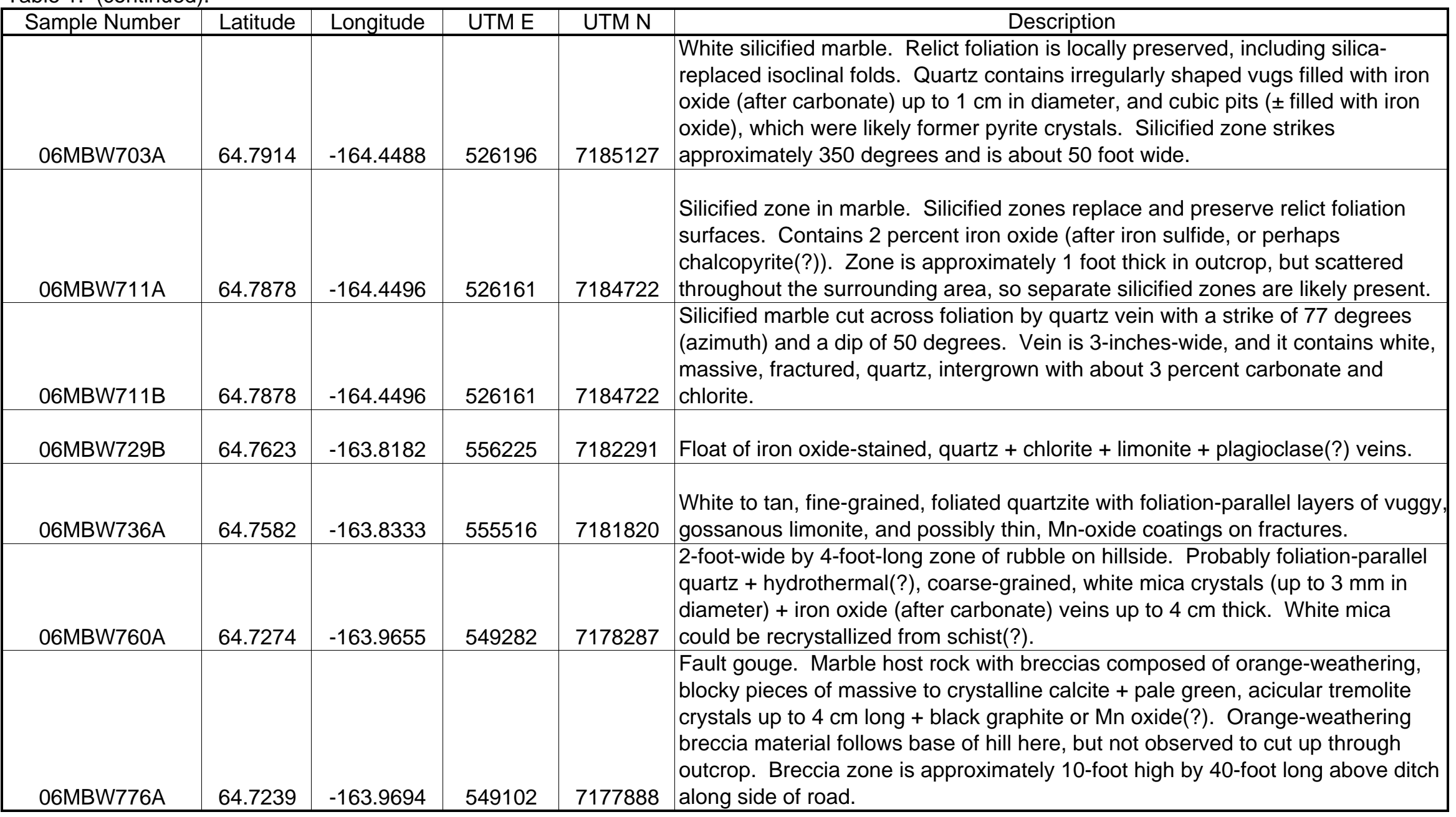


Table 1. (continued).

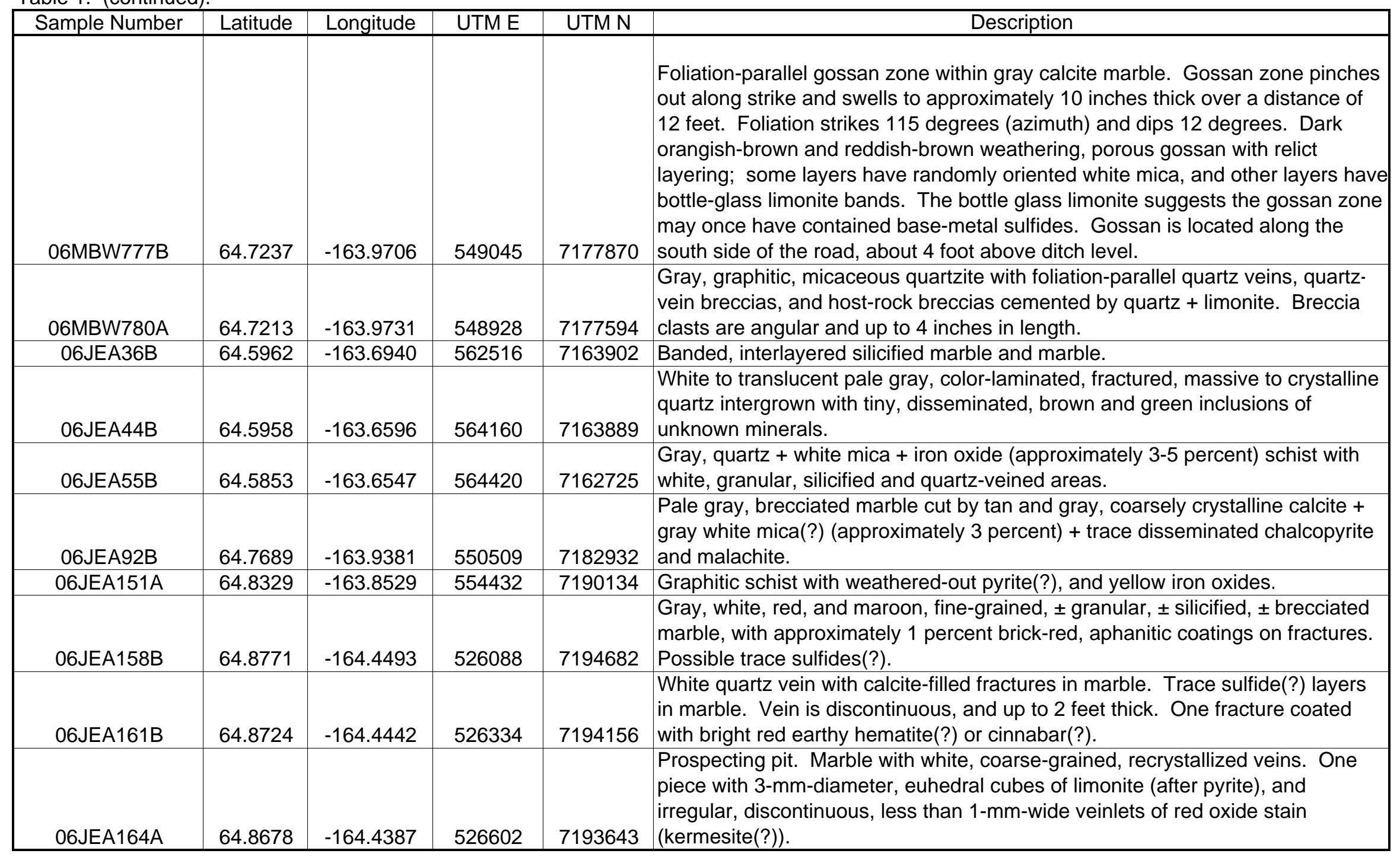


Table 1. (continued).

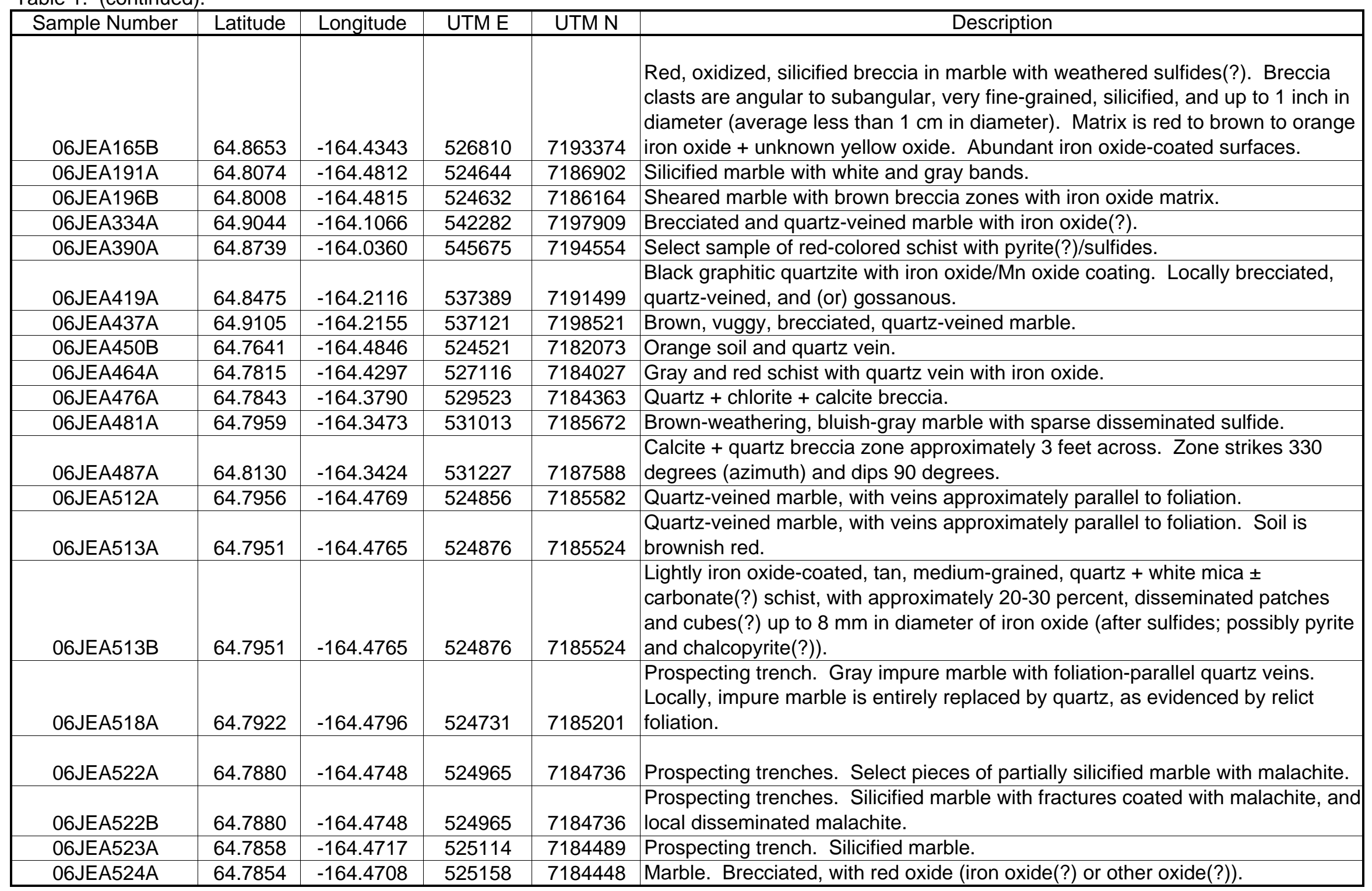


Table 1. (continued).

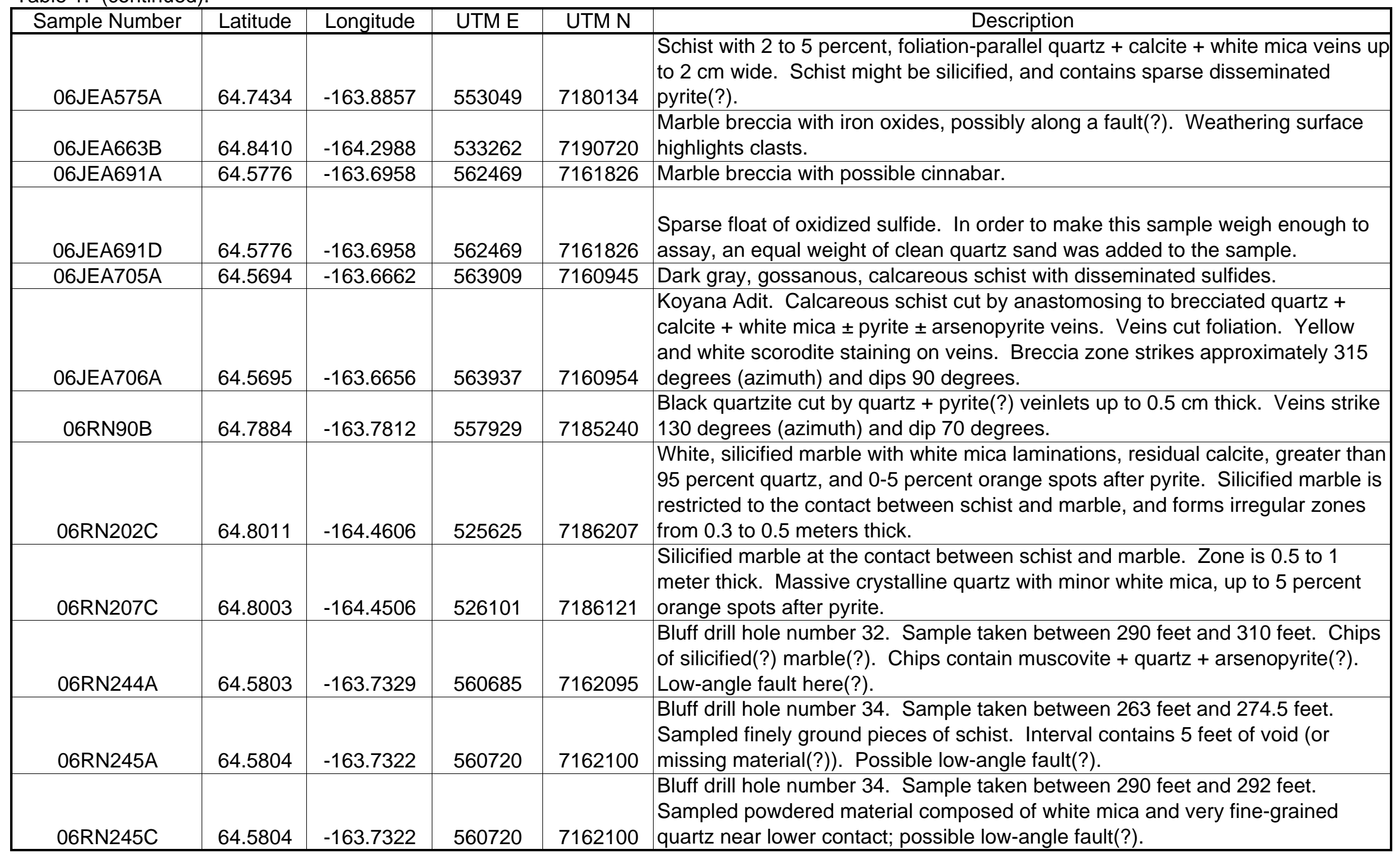


Table 1. (continued).

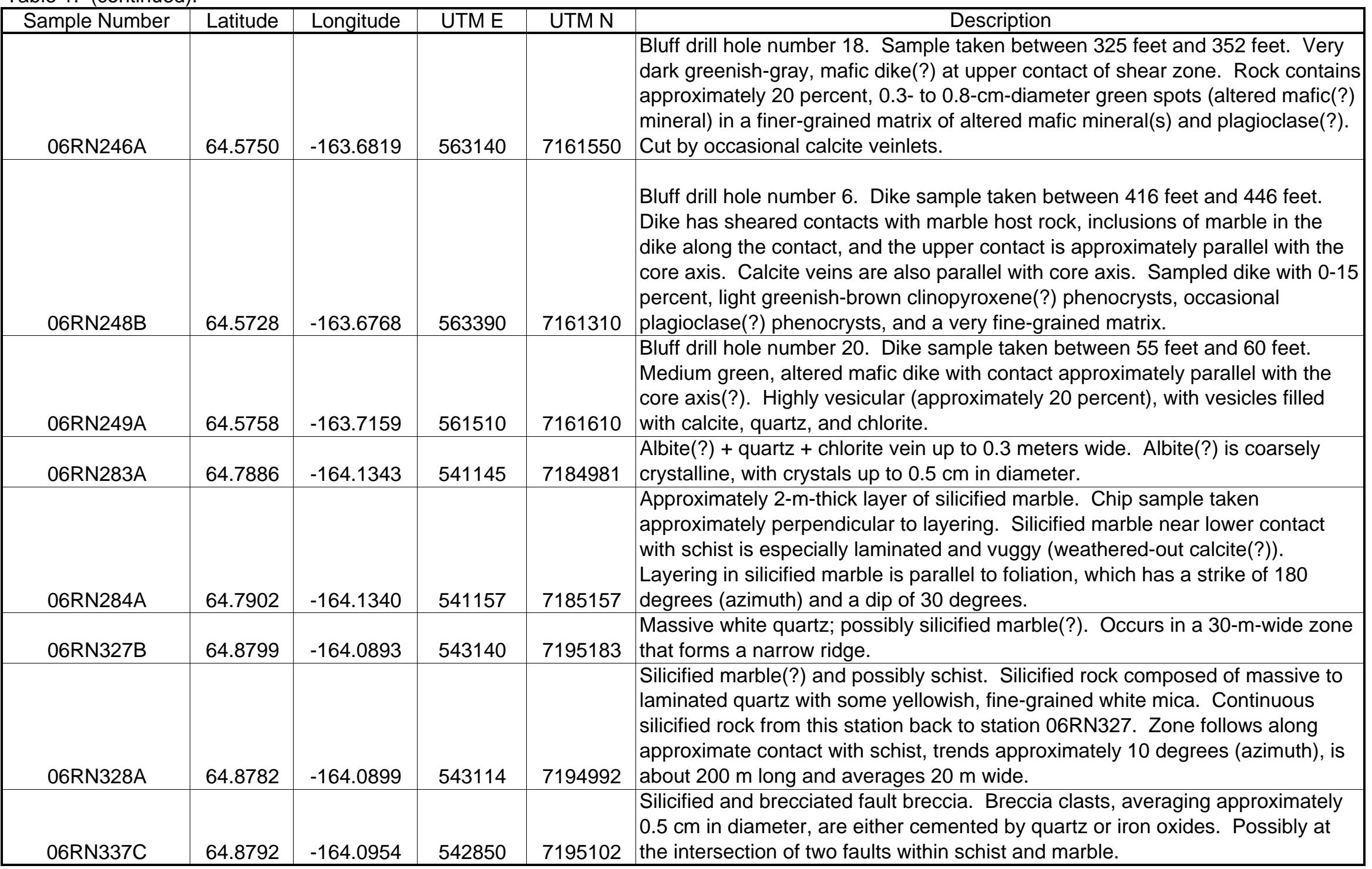


Table 1. (continued).

\begin{tabular}{|c|c|c|c|c|c|}
\hline Sample Number & Latitude & Longitude & UTM E & UTM N & Description \\
\hline 06RN343C & 64.8878 & -164.1088 & 542203 & 7196051 & $\begin{array}{l}\text { Silicified marble at contact between schist and marble. Zone is approximately } \\
10 \mathrm{~m} \text { wide. Silicified marble composed of greater than } 99 \text { percent white, } \\
\text { massive to slightly laminated quartz with less than } 1 \text { percent vugs (former } \\
\text { carbonate or sulfide). Sampled perpendicular to contact. }\end{array}$ \\
\hline 06RN522A & 64.5727 & -163.6773 & 563370 & 7161295 & $\begin{array}{l}\text { Bluff drill hole number } 4 \text {. Dike sample taken between } 199 \text { feet and } 199.5 \text { feet. } \\
\text { Altered mafic dike. }\end{array}$ \\
\hline 06RN556B & 64.7657 & -164.3422 & 531293 & 7182307 & $\begin{array}{l}\text { Quartz } \pm \text { calcite } \pm \text { white mica ( } 0-10 \text { percent }) \pm \text { plagioclase(?) veins along joint } \\
\text { set that has a strike of } 330 \text { degrees (azimuth) and a dip of } 80 \text { degrees. Veins } \\
\text { are } 2-4 \mathrm{~cm} \text { wide. }\end{array}$ \\
\hline 06RN574B & 64.8066 & -164.1576 & 540011 & 7186973 & $\begin{array}{l}\text { Black graphitic quartzite with quartz } \pm \text { iron oxide (after sulfide) veins from } 1-5 \\
\text { mm wide and greater than } 0.3 \mathrm{~m} \text { long. Veins are perpendicular to foliation. } \\
\text { Sample is approximately } 10 \text { percent veins and } 90 \text { percent graphitic quartzite. }\end{array}$ \\
\hline
\end{tabular}


This page has intentionally been left blank. 
Table 2. Trace-element geochemical analyses for rocks collected in the Solomon Quadrangle, Seward Peninsula, Alaska.

Note: - = sample not analyzed for $\mathrm{Au}(+), \mathrm{Cu}(+)$, or $\mathrm{Hg}(+)$; ppm = parts per million; \% = percent; See table 5 for analytical methods.

\begin{tabular}{|c|c|c|c|c|c|c|c|c|c|c|c|c|c|c|c|c|c|c|c|}
\hline Sample Number & $\begin{array}{c}\mathrm{Au} \\
\mathrm{ppm}\end{array}$ & $\begin{array}{l}\mathrm{Au}(+) \\
\mathrm{ppm}\end{array}$ & $\begin{array}{c}\mathrm{Ag} \\
\mathrm{ppm}\end{array}$ & $\begin{array}{l}\mathrm{Al} \\
\% \\
\end{array}$ & $\begin{array}{c}\text { As } \\
\mathrm{ppm}\end{array}$ & $\begin{array}{c}\mathrm{B} \\
\mathrm{ppm}\end{array}$ & $\begin{array}{c}\mathrm{Ba} \\
\mathrm{ppm}\end{array}$ & $\begin{array}{c}\mathrm{Be} \\
\mathrm{ppm}\end{array}$ & $\begin{array}{c}\mathrm{Bi} \\
\mathrm{ppm}\end{array}$ & $\begin{array}{l}\mathrm{Ca} \\
\% \\
\end{array}$ & $\begin{array}{c}\mathrm{Cd} \\
\mathrm{ppm}\end{array}$ & $\begin{array}{c}\text { Co } \\
\mathrm{ppm}\end{array}$ & $\begin{array}{c}\mathrm{Cr} \\
\mathrm{ppm}\end{array}$ & $\begin{array}{c}\mathrm{Cu} \\
\mathrm{ppm}\end{array}$ & $\begin{array}{c}\mathrm{Cu}(+) \\
\% \\
\end{array}$ & $\begin{array}{l}\mathrm{Fe} \\
\% \\
\end{array}$ & $\begin{array}{c}\mathrm{Ga} \\
\mathrm{ppm}\end{array}$ & $\begin{array}{c}\mathrm{Hg} \\
\mathrm{ppm}\end{array}$ & \begin{tabular}{|c|}
$\mathrm{Hg}(+)$ \\
$\mathrm{ppm}$ \\
\end{tabular} \\
\hline 06LF30A & $<0.005$ & - & 0.3 & 0.08 & 56 & $<10$ & 10 & $<0.5$ & $<2$ & 0.01 & $<0.5$ & 4 & 18 & 41 & - & 0.63 & $<10$ & 3 & 3.04 \\
\hline 06LF32C & 0.007 & - & $<0.2$ & 0.15 & 77 & $<10$ & 20 & $<0.5$ & $<2$ & $>25.0$ & $<0.5$ & 6 & 6 & 32 & - & 2.91 & $<10$ & 4 & 3.30 \\
\hline 06LF35C & 0.016 & - & $<0.2$ & 0.12 & 85 & $<10$ & 70 & $<0.5$ & $<2$ & $>25.0$ & 1.7 & 6 & 4 & 13 & - & 2.19 & $<10$ & $<1$ & 0.92 \\
\hline 06LF38A & 0.005 & - & $<0.2$ & 0.06 & 66 & $<10$ & 90 & $<0.5$ & $<2$ & $>25.0$ & 0.5 & 5 & 3 & 7 & - & 1.55 & $<10$ & 2 & 0.69 \\
\hline 06LF43B & $<0.005$ & - & 0.4 & 0.18 & 301 & $<10$ & 10 & $<0.5$ & $<2$ & $>25.0$ & $<0.5$ & 6 & 4 & 103 & - & 11.40 & $<10$ & 1 & 0.82 \\
\hline 06LF44A & $<0.005$ & - & $<0.2$ & 2.39 & 18 & $<10$ & 60 & $<0.5$ & 2 & 0.59 & $<0.5$ & 19 & 34 & 26 & - & 4.61 & 10 & $<1$ & 0.02 \\
\hline 06LF53B & $<0.005$ & - & $<0.2$ & 0.13 & 39 & $<10$ & 20 & $<0.5$ & $<2$ & $>25.0$ & $<0.5$ & 4 & 4 & 8 & - & 0.78 & $<10$ & 1 & 0.26 \\
\hline 06LF56B & $<0.005$ & - & $<0.2$ & 0.22 & 47 & $<10$ & 10 & 0.9 & $<2$ & $>25.0$ & $<0.5$ & 6 & 3 & 9 & - & 2.18 & $<10$ & 1 & 0.06 \\
\hline 06LF64B & $<0.005$ & - & $<0.2$ & 0.71 & 3 & $<10$ & 40 & $<0.5$ & $<2$ & 8.50 & $<0.5$ & 9 & 18 & 18 & - & 2.60 & $<10$ & $<1$ & 0.04 \\
\hline 06LF91B & $<0.005$ & - & $<0.2$ & 2.17 & 18 & $<10$ & 110 & 0.5 & 3 & 0.87 & $<0.5$ & 17 & 25 & 38 & - & 4.21 & 10 & $<1$ & 0.01 \\
\hline 06LF120B & 0.007 & - & $<0.2$ & 2.05 & 8 & $<10$ & 70 & $<0.5$ & $<2$ & 1.23 & $<0.5$ & 14 & 38 & 100 & - & 3.33 & 10 & $<1$ & 0.01 \\
\hline 06LF134A & $<0.005$ & - & $<0.2$ & 1.91 & 4 & $<10$ & 50 & $<0.5$ & $<2$ & 3.00 & $<0.5$ & 9 & 30 & 6 & - & 3.16 & $<10$ & $<1$ & 0.01 \\
\hline 06LF135B & $<0.005$ & - & $<0.2$ & 0.35 & 3 & $<10$ & 20 & $<0.5$ & 3 & 10.80 & $<0.5$ & 6 & 14 & 8 & - & 2.23 & $<10$ & $<1$ & 0.02 \\
\hline 06LF149A & $<0.005$ & - & $<0.2$ & 0.04 & 7 & $<10$ & 10 & $<0.5$ & $<2$ & $>25.0$ & $<0.5$ & 1 & 1 & 2 & - & 0.11 & $<10$ & $<1$ & 0.05 \\
\hline 06LF150A & 0.535 & - & 1.5 & 1.79 & 2660 & $<10$ & 70 & 1.0 & 2 & 0.25 & 2.5 & 13 & 49 & 19 & - & 3.94 & $<10$ & $<1$ & 0.15 \\
\hline 06LF151C & 0.006 & - & $<0.2$ & 0.37 & 95 & $<10$ & 20 & $<0.5$ & $<2$ & $>25.0$ & 0.6 & 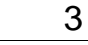 & 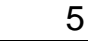 & 34 & - & 0.71 & $<10$ & $<1$ & 0.22 \\
\hline 06LF152B & 0.005 & - & 0.7 & 0.38 & 31 & $<10$ & 500 & $<0.5$ & $<2$ & 0.21 & 0.6 & 2 & 14 & 13 & - & 1.41 & $<10$ & 1 & 0.17 \\
\hline 06LF156B & 0.011 & - & 0.3 & 0.41 & 44 & 10 & 560 & 0.5 & 2 & 0.08 & 1.9 & 7 & 14 & 84 & - & 4.34 & $<10$ & $<1$ & 0.11 \\
\hline 06LF169A & $<0.005$ & - & $<0.2$ & 2.25 & 8 & $<10$ & 10 & $<0.5$ & $<2$ & 2.48 & $<0.5$ & 33 & 116 & 104 & - & 3.21 & $<10$ & $<1$ & 0.01 \\
\hline 06LF178A & $<0.005$ & - & $<0.2$ & 1.94 & 6 & $<10$ & 10 & $<0.5$ & $<2$ & 1.71 & $<0.5$ & 25 & 34 & 285 & - & 4.00 & 10 & $<1$ & 0.01 \\
\hline 06LF180A & $<0.005$ & - & $<0.2$ & 2.14 & 21 & $<10$ & 10 & $<0.5$ & $<2$ & 3.44 & $<0.5$ & 47 & 26 & 60 & - & 5.69 & 10 & $<1$ & 0.02 \\
\hline 06LF189C & $<0.005$ & - & $<0.2$ & 2.88 & 8 & $<10$ & 70 & $<0.5$ & $<2$ & 3.77 & $<0.5$ & 31 & 39 & 49 & - & 6.29 & 10 & 1 & 0.02 \\
\hline 06LF193A & $<0.005$ & - & $<0.2$ & 2.60 & 21 & $<10$ & 70 & 0.5 & $<2$ & 0.20 & $<0.5$ & 15 & 48 & 25 & - & 4.19 & 10 & $<1$ & 0.01 \\
\hline 06LF199A & 0.007 & - & $<0.2$ & 0.83 & 135 & $<10$ & 50 & $<0.5$ & 2 & 6.59 & 0.6 & 10 & 18 & 8 & - & 2.83 & $<10$ & $<1$ & 0.11 \\
\hline 06LF226B & $<0.005$ & - & $<0.2$ & 1.50 & 12 & $<10$ & 10 & $<0.5$ & $<2$ & 10.35 & $<0.5$ & 5 & 18 & 2 & - & 1.37 & $<10$ & $<1$ & 0.01 \\
\hline 06LF288D & $<0.005$ & - & 0.2 & 0.21 & 16 & $<10$ & 100 & $<0.5$ & $<2$ & $>25.0$ & $<0.5$ & 3 & 4 & 10 & - & 1.22 & $<10$ & $<1$ & 0.01 \\
\hline 06LF289B & $<0.005$ & - & $<0.2$ & 0.04 & 109 & $<10$ & 20 & $<0.5$ & $<2$ & 13.25 & $<0.5$ & 2 & 3 & 4 & - & 2.27 & $<10$ & $<1$ & 0.01 \\
\hline 06LF309B & $<0.005$ & - & $<0.2$ & 0.40 & 8 & 10 & 1950 & $<0.5$ & 2 & 0.11 & $<0.5$ & 1 & 18 & 4 & - & 0.82 & $<10$ & $<1$ & 0.16 \\
\hline 06LF323A & 0.089 & - & $<0.2$ & 2.78 & 18 & $<10$ & 60 & $<0.5$ & 2 & 2.89 & $<0.5$ & 36 & 30 & 497 & - & 5.77 & 10 & $<1$ & 0.02 \\
\hline 06LF355A & 0.043 & - & 0.2 & 1.43 & 11 & $<10$ & 270 & $<0.5$ & 2 & 1.27 & 0.5 & 15 & 26 & 270 & - & 2.92 & $<10$ & $<1$ & 0.04 \\
\hline 06LF357A & $<0.005$ & - & $<0.2$ & 0.80 & 27 & $<10$ & 30 & $<0.5$ & $<2$ & $>25.0$ & $<0.5$ & 9 & 10 & 25 & - & 1.74 & $<10$ & $<1$ & 0.07 \\
\hline 06LF357B & $<0.005$ & - & $<0.2$ & 0.35 & 28 & $<10$ & 30 & $<0.5$ & 2 & $>25.0$ & $<0.5$ & 4 & 2 & 16 & - & 2.80 & $<10$ & 1 & $<0.1$ \\
\hline 06LF361B & $<0.005$ & - & $<0.2$ & 2.56 & 15 & $<10$ & 70 & 0.6 & 2 & 0.30 & $<0.5$ & 18 & 41 & 17 & - & 4.15 & 10 & $<1$ & $<0.1$ \\
\hline
\end{tabular}


Table 2. (continued)

\begin{tabular}{|c|c|c|c|c|c|c|c|c|c|c|c|c|c|c|c|c|c|c|c|}
\hline ample Number & $\begin{array}{l}\mathrm{K} \\
\% \\
\end{array}$ & $\begin{array}{c}\mathrm{La} \\
\mathrm{ppm}\end{array}$ & $\begin{array}{c}\mathrm{Mg} \\
\%\end{array}$ & $\begin{array}{c}\mathrm{Mn} \\
\mathrm{ppm}\end{array}$ & $\begin{array}{c}\text { Mo } \\
\text { ppm }\end{array}$ & $\begin{array}{l}\mathrm{Na} \\
\% \\
\end{array}$ & \begin{tabular}{|c|}
$\mathrm{Ni}$ \\
$\mathrm{ppm}$
\end{tabular} & $\begin{array}{l}\mathrm{P} \\
\% \\
\end{array}$ & $\begin{array}{c}\mathrm{Pb} \\
\mathrm{ppm}\end{array}$ & $\begin{array}{l}\mathrm{S} \\
\% \\
\end{array}$ & $\begin{array}{c}\mathrm{Sb} \\
\mathrm{ppm}\end{array}$ & $\begin{array}{c}\mathrm{Sc} \\
\mathrm{ppm}\end{array}$ & $\begin{array}{c}\mathrm{Sr} \\
\mathrm{ppm}\end{array}$ & $\begin{array}{l}\mathrm{Ti} \\
\% \\
\end{array}$ & $\begin{array}{c}\mathrm{TI} \\
\mathrm{ppm}\end{array}$ & $\begin{array}{c}\mathrm{U} \\
\mathrm{ppm}\end{array}$ & $\begin{array}{c}\mathrm{V} \\
\mathrm{ppm}\end{array}$ & $\begin{array}{c}\mathrm{W} \\
\mathrm{ppm}\end{array}$ & $\begin{array}{c}\mathrm{Zn} \\
\mathrm{ppm}\end{array}$ \\
\hline 06LF30A & 0.02 & $<10$ & 0.01 & 43 & 1 & $<0.01$ & 3 & 30 & 10 & $<0.01$ & 12 & $<1$ & 2 & $<0.01$ & $<10$ & $<10$ & 2 & $<10$ & 18 \\
\hline 06LF32C & 0.01 & $<10$ & 3.46 & 564 & $<1$ & 0.01 & 11 & 150 & 9 & $<0.01$ & 1120 & 4 & 43 & $<0.01$ & $<10$ & 10 & 43 & 10 & 26 \\
\hline 06LF35C & 0.03 & $<10$ & 0.29 & 605 & $<1$ & 0.01 & 17 & 130 & 7 & $<0.01$ & 236 & 1 & 26 & $<0.01$ & $<10$ & 10 & 11 & $<10$ & 26 \\
\hline 06LF38A & 0.01 & 10 & 0.22 & 1055 & 1 & $<0.01$ & 6 & 60 & 2 & $<0.01$ & 123 & 1 & 42 & $<0.01$ & $<10$ & 10 & 13 & $<10$ & 42 \\
\hline 06LF43B & 0.02 & $<10$ & 0.10 & 84 & 4 & 0.01 & 65 & 160 & 39 & $<0.01$ & 354 & 1 & 112 & $<0.01$ & $<10$ & 10 & 27 & $<10$ & 32 \\
\hline 06LF44A & 0.22 & 20 & 1.15 & 641 & $<1$ & 0.05 & 64 & 620 & 11 & $<0.01$ & 3 & 6 & 19 & 0.02 & $<10$ & $<10$ & 43 & $<10$ & 122 \\
\hline 06LF53B & 0.02 & $<10$ & 0.08 & 386 & $<1$ & $<0.01$ & 6 & 50 & 4 & $<0.01$ & 71 & 0 & 16 & $<0.01$ & $<10$ & 10 & 12 & $<10$ & 17 \\
\hline 06LF56B & 0.03 & $<10$ & 0.11 & 288 & 2 & $<0.01$ & 14 & 140 & 23 & $<0.01$ & 168 & 1 & 144 & $<0.01$ & $<10$ & 10 & 26 & $<10$ & 70 \\
\hline 06LF64B & 0.22 & 20 & 0.21 & 681 & $<1$ & 0.02 & 29 & 570 & 3 & 0.02 & 4 & 6 & 188 & $<0.01$ & $<10$ & $<10$ & 16 & $<10$ & 45 \\
\hline 06LF91B & 0.32 & 30 & 1.54 & 394 & 3 & 0.02 & 46 & 520 & 8 & 0.47 & 3 & 5 & 37 & 0.01 & $<10$ & $<10$ & 30 & $<10$ & 95 \\
\hline 06LF120B & 0.36 & 20 & 1.38 & 767 & $<1$ & 0.03 & 36 & 670 & 2 & $<0.01$ & 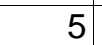 & 5 & 34 & 0.04 & $<10$ & $<10$ & 44 & $<10$ & 62 \\
\hline 06LF & 0.33 & 20 & 0.62 & 446 & $<1$ & 0.02 & 42 & 870 & 2 & $<0.01$ & 3 & 4 & 71 & 0.01 & $<10$ & $<10$ & 21 & $<10$ & 65 \\
\hline 06LF135B & 0.08 & $<10$ & 0.16 & 504 & $<1$ & 0.02 & 22 & 310 & 21 & $<0.01$ & 3 & $\sqrt{3}$ & 545 & $<0.01$ & 10 & $<10$ & 10 & $<10$ & 79 \\
\hline 06LF1 & 0.01 & $<10$ & 0.27 & 106 & $<1$ & 0.01 & $<1$ & 20 & 2 & $<0.01$ & 3 & $<1$ & 780 & $<0.01$ & 10 & 10 & 3 & $<10$ & \\
\hline $0 A$ & 0.21 & 40 & 0.61 & 457 & 1 & 0.01 & 40 & 470 & 31 & $<0.01$ & 37 & 0 & 7 & 0.01 & $<10$ & $<10$ & 62 & 650 & 118 \\
\hline LC & 0.07 & 10 & 0.20 & 122 & 8 & 0.06 & 47 & 300 & 10 & $<0.01$ & 13 & 2 & 665 & $<0.01$ & $<10$ & 10 & 57 & $<10$ & 69 \\
\hline 06LF152B & 0.10 & 10 & 0.03 & 117 & 18 & $<0.01$ & 25 & 310 & 5 & $<0.01$ & 20 & 1 & 11 & $<0.01$ & $<10$ & $<10$ & 52 & $<10$ & 72 \\
\hline 06LF156B & 0.13 & $<10$ & 0.04 & 102 & 17 & 0.01 & 85 & 540 & 8 & 0.07 & 245 & 2 & 17 & $<0.01$ & $<10$ & 10 & 24 & $<10$ & 258 \\
\hline 06LF169A & 0.01 & $<10$ & 2.26 & 727 & $<1$ & 0.04 & 59 & 340 & 5 & 0.11 & 3 & 7 & 148 & 0.26 & $<10$ & $<10$ & 68 & $<10$ & 48 \\
\hline 06LF178A & .01 & $<10$ & 1.38 & 449 & $<1$ & 0.07 & 24 & 990 & $<2$ & 0.06 & 2 & 7 & 34 & 0.40 & $<10$ & $<10$ & 87 & $<10$ & 57 \\
\hline 06LF180A & $<0.01$ & $<10$ & 2.02 & 561 & 1 & 0.07 & 31 & 4200 & $<2$ & 0.54 & 2 & 8 & 185 & 0.18 & $<10$ & $<10$ & 149 & $<10$ & 82 \\
\hline 06LF189C & 0.12 & $<10$ & 1.88 & 777 & 1 & 0.05 & 41 & 1690 & 2 & 0.14 & $<2$ & 5 & 319 & 0.66 & $<10$ & $<10$ & 158 & $<10$ & 106 \\
\hline 06LF193A & 0.30 & 30 & 1.34 & 596 & $<1$ & 0.02 & 60 & 750 & 4 & $<0.01$ & 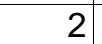 & 5 & 9 & 0.02 & $<10$ & $<10$ & 47 & $<10$ & 66 \\
\hline 06LF199A & 0.28 & 20 & 0.12 & 744 & $<1$ & 0.02 & 32 & 540 & 7 & $<0.01$ & 76 & 4 & 138 & $<0.01$ & $<10$ & $<10$ & 17 & $<10$ & 92 \\
\hline 06LF226B & 0.01 & $<10$ & 5.06 & 159 & $<1$ & 0.01 & 14 & 170 & 5 & $<0.01$ & $<2$ & 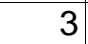 & 229 & 0.06 & $<10$ & $<10$ & 23 & $<10$ & 13 \\
\hline 06LF288D & 0.06 & $<10$ & 0.33 & 214 & 1 & 0.01 & 36 & 230 & 3 & $<0.01$ & 41 & 2 & 604 & $<0.01$ & $<10$ & 10 & 13 & $<10$ & 98 \\
\hline 06LF289B & 0.01 & $<10$ & 2.60 & 554 & $<1$ & $<0.01$ & 7 & 40 & $<2$ & $<0.01$ & 4 & $\perp$ & 284 & $<0.01$ & $<10$ & $<10$ & 3 & $<10$ & 11 \\
\hline 06LF309B & 0.08 & $<10$ & 0.04 & 56 & 11 & 0.03 & 3 & 70 & 21 & 0.02 & 5 & $\perp$ & 5 & $<0.01$ & $<10$ & $<10$ & 50 & $<10$ & 6 \\
\hline 06LF323A & 0.03 & $<10$ & 2.44 & 422 & 1 & 0.05 & 49 & 3590 & $<2$ & 0.14 & $<2$ & 8 & 131 & 0.33 & $<10$ & $<10$ & 164 & $<10$ & 97 \\
\hline 06LF355A & 0.22 & 10 & 0.86 & 210 & 2 & 0.03 & 38 & 2390 & 3 & 0.07 & 2 & 4 & 80 & 0.19 & $<10$ & $<10$ & 95 & $<10$ & 100 \\
\hline 06LF357A & 0.09 & $<10$ & 0.25 & 243 & $<1$ & 0.01 & 21 & 760 & 3 & $<0.01$ & 45 & 5 & 355 & $<0.01$ & $<10$ & 10 & 28 & $<10$ & 21 \\
\hline 06LF357B & 0.03 & $<10$ & 0.16 & 343 & $<1$ & 0.01 & 6 & 660 & 6 & $<0.01$ & 31 & 4 & 599 & $<0.01$ & $<10$ & 10 & 16 & $<10$ & 27 \\
\hline 06LF361B & 0.39 & 30 & 0.92 & 438 & 1 & 0.03 & 49 & 650 & 3 & 0.01 & 3 & 4 & 12 & 0.01 & $<10$ & $<10$ & 44 & $<10$ & 48 \\
\hline
\end{tabular}


Table 2. (continued).

\begin{tabular}{|c|c|c|c|c|c|c|c|c|c|c|c|c|c|c|c|c|c|c|c|}
\hline Sample Number & $\begin{array}{c}\mathrm{Au} \\
\mathrm{ppm}\end{array}$ & $\begin{array}{c}\mathrm{Au}(+) \\
\mathrm{ppm}\end{array}$ & $\begin{array}{c}\mathrm{Ag} \\
\mathrm{ppm}\end{array}$ & $\begin{array}{l}\mathrm{Al} \\
\% \\
\end{array}$ & $\begin{array}{c}\text { As } \\
\text { ppm }\end{array}$ & $\begin{array}{c}\text { B } \\
\text { ppm }\end{array}$ & $\begin{array}{c}\mathrm{Ba} \\
\mathrm{ppm}\end{array}$ & $\begin{array}{c}\mathrm{Be} \\
\mathrm{ppm}\end{array}$ & $\begin{array}{c}\mathrm{Bi} \\
\text { ppm }\end{array}$ & $\begin{array}{l}\mathrm{Ca} \\
\% \\
\end{array}$ & $\begin{array}{c}\text { Cd } \\
\text { ppm }\end{array}$ & $\begin{array}{c}\text { Co } \\
\text { ppm }\end{array}$ & $\begin{array}{c}\mathrm{Cr} \\
\mathrm{ppm}\end{array}$ & $\begin{array}{c}\mathrm{Cu} \\
\mathrm{ppm}\end{array}$ & $\begin{array}{c}\mathrm{Cu}(+) \\
\%\end{array}$ & $\begin{array}{l}\mathrm{Fe} \\
\% \\
\end{array}$ & $\begin{array}{c}\mathrm{Ga} \\
\mathrm{ppm}\end{array}$ & $\begin{array}{c}\mathrm{Hg} \\
\mathrm{ppm}\end{array}$ & \begin{tabular}{|c}
$\mathrm{Hg}(+)$ \\
$\mathrm{ppm}$ \\
\end{tabular} \\
\hline 06LF362A & 0.423 & - & 0.3 & 2.37 & 254 & $<10$ & 60 & 0.5 & 2 & 0.85 & $<0.5$ & 16 & 60 & 8 & - & 3.93 & 10 & $<1$ & $<0.1$ \\
\hline 06LF372B & $<0.005$ & - & $<0.2$ & 1.49 & 13 & $<10$ & 50 & 0.6 & 2 & 7.22 & $<0.5$ & 12 & 33 & 29 & - & 3.75 & $<10$ & $<1$ & 0.08 \\
\hline 06LF373B & $<0.005$ & - & $<0.2$ & 0.66 & 2 & $<10$ & 60 & $<0.5$ & 2 & 10.85 & 0.5 & 5 & 9 & 5 & - & 3.94 & $<10$ & $<1$ & 0.02 \\
\hline 06LF382A & $<0.005$ & - & $<0.2$ & 0.84 & 39 & $<10$ & 50 & $<0.5$ & $<2$ & 8.19 & $<0.5$ & 10 & 18 & 24 & - & 3.10 & $<10$ & 1 & 0.08 \\
\hline 06LF393A & $<0.005$ & - & 0.8 & 0.41 & 27 & $<10$ & 90 & $<0.5$ & 2 & 0.05 & 0.7 & 5 & 19 & 55 & - & 2.30 & $<10$ & $<1$ & 0.06 \\
\hline 06LF400A & 0.008 & - & $<0.2$ & 2.35 & 17 & $<10$ & 10 & $<0.5$ & 3 & 2.31 & $<0.5$ & 44 & 12 & 215 & - & 6.90 & 10 & $<1$ & 0.02 \\
\hline 06LF407A & $<0.005$ & - & 0.3 & 0.17 & 36 & $<10$ & 120 & $<0.5$ & 2 & 0.01 & $<0.5$ & 1 & 56 & 56 & - & 4.88 & $<10$ & $<1$ & 0.08 \\
\hline 06LF408B & 0.005 & - & 0.2 & 0.17 & 14 & $<10$ & 160 & $<0.5$ & $<2$ & $<0.01$ & 0.6 & 4 & 35 & 26 & - & 1.07 & $<10$ & $<1$ & 0.09 \\
\hline 06LF409B & $<0.005$ & - & 0.4 & 0.58 & 25 & $<10$ & 40 & $<0.5$ & 6 & 0.01 & 0.9 & 1 & 21 & 427 & - & 32.80 & $<10$ & $<1$ & 0.10 \\
\hline 06LF413B & $<0.005$ & - & 0.3 & 0.10 & 19 & $<10$ & 10 & $<0.5$ & 2 & 0.01 & 2.0 & 33 & 51 & 169 & - & 11.00 & $<10$ & 1 & 0.10 \\
\hline 06LF423B & $<0.005$ & - & 0.5 & 0.27 & 22 & $<10$ & 170 & $<0.5$ & $<2$ & 0.02 & 2.3 & 4 & 39 & 58 & - & 1.66 & $<10$ & $<1$ & 0.09 \\
\hline 06LF442B & $<0.005$ & - & 0.2 & 0.10 & 5 & $<10$ & 160 & $<0.5$ & $<2$ & 0.01 & $<0.5$ & $<1$ & 62 & 3 & - & 0.17 & $<10$ & 1 & 0.06 \\
\hline 06LF449A & $<0.005$ & - & $<0.2$ & 1.44 & 107 & $<10$ & 30 & $<0.5$ & 2 & 21.50 & $<0.5$ & 27 & 176 & 80 & - & 4.13 & $<10$ & $<1$ & $<0.1$ \\
\hline 06LF450A & 0.006 & - & $<0.2$ & 0.05 & 43 & $<10$ & 20 & $<0.5$ & $<2$ & 0.57 & $<0.5$ & 9 & 58 & 10 & - & 1.30 & $<10$ & $<1$ & 0.02 \\
\hline 06LF457A & $<0.005$ & - & 0.4 & 0.55 & $<2$ & $<10$ & 40 & $<0.5$ & $<2$ & 3.18 & 0.5 & 10 & 27 & 34 & - & 2.27 & $<10$ & $<1$ & 0.04 \\
\hline 06LF489A & $<0.005$ & - & $<0.2$ & 1.64 & 2 & $<10$ & 70 & 0.5 & $<2$ & 1.83 & 0.6 & 14 & 75 & 62 & - & 2.41 & $<10$ & 1 & 0.08 \\
\hline 06LF492A & $<0.005$ & - & $<0.2$ & 0.23 & 5 & $<10$ & 120 & $<0.5$ & $<2$ & 7.71 & 1.0 & 4 & 24 & 17 & - & 1.40 & $<10$ & 1 & 0.22 \\
\hline 06LF492B & $<0.005$ & - & $<0.2$ & 0.15 & 7 & $<10$ & 200 & $<0.5$ & $<2$ & 24.40 & $<0.5$ & 3 & 12 & 10 & - & 1.21 & $<10$ & $<1$ & 0.04 \\
\hline 06LF495A & $<0.005$ & - & 0.2 & 1.67 & 18 & $<10$ & 40 & $<0.5$ & 2 & 1.50 & $<0.5$ & 29 & 30 & 59 & - & 4.28 & 10 & 1 & 0.32 \\
\hline 06LF499B & $<0.005$ & - & $<0.2$ & 0.20 & 23 & $<10$ & 40 & $<0.5$ & $<2$ & $>25.0$ & $<0.5$ & 3 & 7 & 2370 & - & 1.61 & $<10$ & $<1$ & 0.05 \\
\hline 06LF501B & $<0.005$ & - & $<0.2$ & 0.03 & 2 & $<10$ & $<10$ & $<0.5$ & 2 & 0.56 & $<0.5$ & $<1$ & 33 & 48 & - & 0.15 & $<10$ & $<1$ & 0.01 \\
\hline 06LF501C & $<0.005$ & - & $<0.2$ & 1.28 & 4 & $<10$ & 40 & 0.9 & 4 & 1.50 & 0.5 & 51 & 39 & 101 & - & 8.25 & $<10$ & $<1$ & 0.14 \\
\hline 06LF508A & $<0.005$ & - & 0.3 & 0.78 & 17 & $<10$ & 130 & $<0.5$ & $<2$ & 0.40 & 1.7 & 12 & 35 & 71 & - & 3.32 & $<10$ & 1 & 0.26 \\
\hline 06LF534B & $<0.005$ & - & 0.3 & 0.12 & 29 & $<10$ & 10 & $<0.5$ & $<2$ & $>25.0$ & 0.9 & 7 & 6 & 10 & - & 0.49 & $<10$ & $<1$ & 0.10 \\
\hline 06LF535B & $<0.005$ & - & $<0.2$ & 0.07 & 16 & $<10$ & 10 & $<0.5$ & $<2$ & $>25.0$ & $<0.5$ & 2 & 1 & 4 & - & 0.28 & $<10$ & $<1$ & 0.21 \\
\hline 06LF539A & 0.024 & - & 2.3 & 0.07 & 103 & $<10$ & 10 & $<0.5$ & $<2$ & 0.66 & 0.5 & 2 & 46 & 16 & - & 0.30 & $<10$ & $<1$ & 0.48 \\
\hline 06LF543B & $<0.005$ & - & 0.3 & 0.12 & 141 & $<10$ & 10 & $<0.5$ & $<2$ & $>25.0$ & 0.8 & 2 & 3 & 4 & - & 0.76 & $<10$ & $<1$ & 0.68 \\
\hline 06LF550B & 0.016 & - & $<0.2$ & 0.11 & 169 & $<10$ & 20 & $<0.5$ & 2 & $>25.0$ & $<0.5$ & 5 & 3 & 6 & - & 1.98 & $<10$ & 1 & 0.77 \\
\hline 06LF562A & 0.006 & - & $<0.2$ & 0.62 & 73 & $<10$ & 30 & 1.2 & 7 & 0.85 & 1.4 & 12 & 8 & 154 & - & 31.40 & $<10$ & $<1$ & 0.10 \\
\hline 06LF585B & $<0.005$ & - & $<0.2$ & 1.86 & 19 & $<10$ & 10 & $<0.5$ & $<2$ & 1.61 & $<0.5$ & 35 & 17 & 54 & - & 5.97 & 10 & $<1$ & 0.04 \\
\hline 06Z28B & 6.080 & - & 0.9 & 0.06 & $>10000$ & $<10$ & $<10$ & $<0.5$ & 8 & 0.08 & $<0.5$ & 1 & 42 & 4 & - & 6.01 & $<10$ & 1 & 0.10 \\
\hline 06Z36A & 0.047 & - & 0.2 & 0.02 & 762 & $<10$ & $<10$ & $<0.5$ & $<2$ & $>25.0$ & $<0.5$ & 2 & 3 & 2 & - & 0.15 & $<10$ & $<1$ & 0.05 \\
\hline 06Z47B & $<0.005$ & - & $<0.2$ & 1.34 & 34 & $<10$ & 10 & $<0.5$ & 2 & 2.23 & $<0.5$ & 5 & 49 & 8 & - & 2.72 & $<10$ & $<1$ & 0.23 \\
\hline 06Z100B & $<0.005$ & - & $<0.2$ & 1.53 & 21 & $<10$ & 40 & $<0.5$ & 3 & 16.10 & 0.6 & 12 & 34 & 13 & - & 3.51 & $<10$ & $<1$ & 0.38 \\
\hline
\end{tabular}


Table 2. (continued)

\begin{tabular}{|c|c|c|c|c|c|c|c|c|c|c|c|c|c|c|c|c|c|c|c|}
\hline Sample Number & $\begin{array}{l}\mathrm{K} \\
\%\end{array}$ & $\begin{array}{c}\mathrm{La} \\
\mathrm{ppm}\end{array}$ & $\begin{array}{c}\mathrm{Mg} \\
\%\end{array}$ & $\begin{array}{c}\mathrm{Mn} \\
\mathrm{ppm}\end{array}$ & $\begin{array}{c}\text { Mo } \\
\text { ppm }\end{array}$ & $\begin{array}{c}\mathrm{Na} \\
\%\end{array}$ & $\begin{array}{c}\mathrm{Ni} \\
\mathrm{ppm}\end{array}$ & $\begin{array}{l}\mathrm{P} \\
\%\end{array}$ & $\begin{array}{c}\mathrm{Pb} \\
\mathrm{ppm}\end{array}$ & $\begin{array}{l}\mathrm{S} \\
\%\end{array}$ & $\begin{array}{c}\mathrm{Sb} \\
\mathrm{ppm}\end{array}$ & $\begin{array}{c}\text { Sc } \\
\text { ppm }\end{array}$ & $\begin{array}{c}\mathrm{Sr} \\
\mathrm{ppm}\end{array}$ & $\begin{array}{l}\mathrm{Ti} \\
\%\end{array}$ & $\begin{array}{c}\mathrm{TI} \\
\mathrm{ppm}\end{array}$ & $\begin{array}{c}\mathrm{U} \\
\mathrm{ppm}\end{array}$ & $\begin{array}{c}\mathrm{V} \\
\mathrm{ppm}\end{array}$ & $\begin{array}{c}\mathrm{W} \\
\mathrm{ppm}\end{array}$ & $\begin{array}{c}\mathrm{Zn} \\
\mathrm{ppm}\end{array}$ \\
\hline 06LF362A & 0.29 & 30 & 1.02 & 470 & $<1$ & 0.04 & 46 & 630 & 8 & 0.01 & 6 & 6 & 13 & 0.01 & $<10$ & $<10$ & 59 & $<10$ & 98 \\
\hline oLF372B & 0.23 & 20 & 1.19 & 15 & $<1$ & 0.01 & 47 & 650 & 10 & 0.15 & 4 & 8 & 153 & 0.01 & $<10$ & $<10$ & 32 & 10 & 5 \\
\hline LF373B & 0.15 & 40 & 3.50 & 280 & 1 & 0.0 & 21 & 1050 & & 0.07 & 3 & & 162 & 0.01 & $<10$ & $<10$ & 15 & $<10$ & 33 \\
\hline 06LF382A & 0.25 & 10 & 1.13 & 582 & $<1$ & 0.01 & 27 & 560 & $\varepsilon$ & 0.37 & 20 & 5 & 251 & $<0.01$ & $<10$ & $<10$ & 18 & $<10$ & 52 \\
\hline 06LF393A & 0.18 & 10 & 0.04 & 93 & 56 & $<0.01$ & 39 & 350 & 28 & 0.05 & & 2 & 15 & $<0.01$ & $<10$ & 10 & 72 & $<10$ & 73 \\
\hline 06LF400A & 0.02 & $<10$ & 1.75 & 739 & $<1$ & 0.10 & 28 & 1170 & $<2$ & 0.80 & 3 & & 74 & 0.54 & $<10$ & $<10$ & 130 & $<10$ & 67 \\
\hline 06LF407A & 0.05 & 20 & 0.01 & 8 & 29 & $<0.01$ & 9 & 810 & 32 & 0.03 & 5 & & 20 & $<0.01$ & $<10$ & $<10$ & 68 & $<10$ & Is \\
\hline 06LF408B & 0.07 & $<10$ & 0.01 & 16 & 9 & $<0.01$ & 16 & 200 & 10 & $<0.01$ & 6 & $<1$ & 5 & $<0.01$ & $<10$ & $<10$ & 16 & $<10$ & 01 \\
\hline 06LF409B & 0.02 & $<10$ & 0.01 & 296 & 38 & $<0.01$ & 118 & 2060 & $<2$ & 0.36 & 7 & & 15 & $<0.01$ & $<10$ & $<10$ & 227 & $<10$ & 657 \\
\hline 06LF413B & 0.01 & $<10$ & 0.01 & 90 & 11 & $<0.01$ & 221 & 490 & 3 & 0.01 & 54 & & 3 & $<0.01$ & $<10$ & 10 & 8 & $<10$ & 316 \\
\hline 06LF423B & 0.11 & 10 & 0.02 & 17 & 34 & $<0.01$ & 92 & 370 & 5 & 0.03 & 4 & 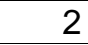 & 25 & $<0.01$ & $<10$ & 10 & 44 & $<10$ & 263 \\
\hline 06LF442B & 0.04 & 10 & 0.01 & 9 & 4 & $<0.01$ & 4 & 10 & 3 & 0.01 & $<2$ & $<1$ & 3 & $<0.01$ & $<10$ & $<10$ & 8 & $<10$ & 3 \\
\hline 06LF449A & 0.08 & 10 & 1.60 & 937 & $<1$ & $<0.01$ & 169 & 1310 & $<2$ & $<0.01$ & 109 & 11 & 340 & $<0.01$ & $<10$ & $<10$ & 53 & $<10$ & 37 \\
\hline 06LF450A & 0.01 & $<10$ & 0.12 & 1080 & 1 & $<0.01$ & 36 & 30 & 2 & 0.11 & 4 & & 5 & $<0.01$ & $<10$ & $<10$ & 2 & $<10$ & 8 \\
\hline 06LF457A & 14 & 20 & 0.50 & 165 & 1 & $<0.01$ & 27 & 530 & 6 & 0.45 & $<2$ & & 103 & $<0.01$ & $<10$ & $<10$ & 5 & $<10$ & 38 \\
\hline 06LF489A & 14 & 10 & 1.56 & 1025 & $<1$ & 0.02 & 51 & 670 & 30 & 0.01 & 3 & 5 & 70 & 0.03 & $<10$ & $<10$ & 39 & $<10$ & 71 \\
\hline 06LF492A & 06 & $<10$ & 0.14 & 182 & $<1$ & $<0.01$ & 26 & 260 & 2 & 0.01 & 3 & 4 & 211 & $<0.01$ & $<10$ & $<10$ & 32 & $<10$ & 51 \\
\hline 06LF492B & 0.06 & $<10$ & 0.41 & 243 & $<1$ & 0.01 & 14 & 200 & 5 & $<0.01$ & 3 & 3 & 944 & $<0.01$ & $<10$ & 10 & 7 & $<10$ & 21 \\
\hline LF495A & 0.01 & $<10$ & 1.41 & 310 & $<1$ & 0.03 & 32 & 3800 & 8 & 0.05 & $<2$ & 4 & 89 & 0.19 & $<10$ & $<10$ & 89 & $<10$ & 63 \\
\hline LLF499B & 0.03 & $<10$ & 0.76 & 955 & $<1$ & 0.01 & 4 & 360 & 2 & 0.01 & 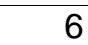 & 2 & 619 & 0.01 & 10 & 10 & 5 & $<10$ & 5 \\
\hline LLF501B & 0.01 & $<10$ & 0.01 & 19 & $<1$ & $<0.01$ & 3 & 20 & $<2$ & $<0.01$ & $<2$ & $<1$ & 14 & $<0.01$ & $<10$ & $<10$ & 1 & $<10$ & $<2$ \\
\hline LF501C & 0.02 & 10 & 0.27 & 959 & 1 & $<0.01$ & 91 & 1440 & $<2$ & 0.02 & 13 & 28 & 39 & 0.01 & $<10$ & $<10$ & 198 & $<10$ & 113 \\
\hline LF508A & 0.07 & 10 & 0.38 & 408 & 4 & $<0.01$ & 82 & 760 & 7 & 0.13 & 7 & 0 & 22 & $<0.01$ & $<10$ & $<10$ & 32 & $<10$ & 213 \\
\hline LF534B & 0.01 & 10 & 8.19 & 352 & 2 & 0.01 & 11 & 90 & 97 & $<0.01$ & 127 & 2 & 213 & 0.01 & $<10$ & 10 & 7 & $<10$ & 85 \\
\hline 06LF535B & 0.01 & $<10$ & 0.80 & 190 & $<1$ & $<0.01$ & 4 & 30 & 2 & $<0.01$ & 32 & $<1$ & 100 & $<0.01$ & $<10$ & 10 & 5 & $<10$ & 16 \\
\hline 06LF539A & 0.03 & $<10$ & 0.01 & 23 & $<1$ & $<0.01$ & 4 & 30 & 78 & $<0.01$ & 101 & $<1$ & 1 & $<0.01$ & $<10$ & $<10$ & 2 & $<10$ & 39 \\
\hline 06LF543B & 03 & $<10$ & 0.24 & 175 & $<1$ & 0.01 & 7 & 100 & 7 & $<0.01$ & 32 & 2 & 10 & $<0.01$ & $<10$ & 1 & 1 & $<10$ & 121 \\
\hline 06LF550B & 0.01 & $<10$ & 0.36 & 985 & 2 & 0.01 & 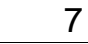 & 60 & 5 & $<0.01$ & 107 & & 512 & $<0.01$ & $<10$ & 10 & 10 & $<10$ & 14 \\
\hline 06LF562A & 09 & 20 & 0.10 & 183 & 44 & 0.01 & 151 & 2810 & 23 & 0.01 & 4 & & 14 & $<0.01$ & $<10$ & 10 & 15 & $<10$ & 408 \\
\hline 06LF585B & 02 & 10 & 1.1 & 530 & 1 & 0.02 & 29 & 3720 & & 0.26 & 3 & & 165 & 0.09 & $<10$ & $<10$ & 105 & $<10$ & 98 \\
\hline 06Z28B & 3 & $<10$ & 0.02 & 14 & 5 & $<0.01$ & 2 & 60 & 15 & 0.56 & 215 & & 21 & $<0.01$ & $<10$ & $<10$ & $?$ & 10 & y \\
\hline 06Z36A & $<0.01$ & $<10$ & 1.63 & 259 & $<1$ & 0.01 & $<1$ & 30 & $<2$ & $<0.01$ & 47 & $<1$ & 234 & $<0.01$ & $<10$ & 10 & 4 & $<10$ & 4 \\
\hline 06Z47B & 0.05 & 10 & 1.67 & 222 & 1 & 0.01 & 24 & 860 & 2 & 0.01 & 2 & 3 & 59 & $<0.01$ & $<10$ & $<10$ & 27 & $<10$ & 26 \\
\hline 66Z100B & 0.02 & 10 & 1.13 & 19 & & 0.01 & 39 & 480 & 12 & 0.31 & & & 583 & $<0.01$ & 10 & $\leqslant 10$ & 15 & $=10$ & \\
\hline
\end{tabular}


Table 2. (continued).

\begin{tabular}{|c|c|c|c|c|c|c|c|c|c|c|c|c|c|c|c|c|c|c|c|}
\hline Sample Number & $\begin{array}{c}\mathrm{Au} \\
\mathrm{ppm}\end{array}$ & $\begin{array}{c}\mathrm{Au}(+) \\
\mathrm{ppm}\end{array}$ & $\begin{array}{c}\mathrm{Ag} \\
\mathrm{ppm}\end{array}$ & $\begin{array}{l}\mathrm{Al} \\
\% \\
\end{array}$ & $\begin{array}{c}\text { As } \\
\text { ppm }\end{array}$ & $\begin{array}{c}\text { B } \\
\text { ppm }\end{array}$ & $\begin{array}{c}\mathrm{Ba} \\
\mathrm{ppm}\end{array}$ & $\begin{array}{c}\mathrm{Be} \\
\mathrm{ppm}\end{array}$ & $\begin{array}{c}\mathrm{Bi} \\
\text { ppm }\end{array}$ & $\begin{array}{l}\mathrm{Ca} \\
\% \\
\end{array}$ & $\begin{array}{c}\text { Cd } \\
\text { ppm }\end{array}$ & $\begin{array}{c}\text { Co } \\
\text { ppm }\end{array}$ & $\begin{array}{c}\mathrm{Cr} \\
\mathrm{ppm}\end{array}$ & $\begin{array}{c}\mathrm{Cu} \\
\mathrm{ppm}\end{array}$ & $\begin{array}{c}\mathrm{Cu}(+) \\
\%\end{array}$ & $\begin{array}{l}\mathrm{Fe} \\
\% \\
\end{array}$ & $\begin{array}{c}\mathrm{Ga} \\
\mathrm{ppm}\end{array}$ & $\begin{array}{c}\mathrm{Hg} \\
\mathrm{ppm}\end{array}$ & \begin{tabular}{|c}
$\mathrm{Hg}(+)$ \\
$\mathrm{ppm}$ \\
\end{tabular} \\
\hline 06Z108A & $<0.005$ & - & $<0.2$ & 0.80 & 19 & $<10$ & 20 & $<0.5$ & $<2$ & 3.38 & $<0.5$ & 12 & 40 & 9 & - & 2.08 & $<10$ & $<1$ & 0.37 \\
\hline 06Z125A & $<0.005$ & - & 0.2 & 0.04 & 17 & $<10$ & 10 & $<0.5$ & $<2$ & $>25.0$ & $<0.5$ & 1 & 8 & 4 & - & 0.37 & $<10$ & $<1$ & 0.52 \\
\hline 06Z143B & 0.005 & - & $<0.2$ & 0.10 & 28 & $<10$ & 10 & $<0.5$ & $<2$ & 7.65 & $<0.5$ & 5 & 31 & 30 & - & 1.19 & $<10$ & $<1$ & 0.18 \\
\hline 06Z151A & 8.38 & - & 39.4 & 0.11 & $>10000$ & $<10$ & 340 & $<0.5$ & 45 & 2.07 & 7.9 & 8 & 65 & 1040 & - & 19.10 & $<10$ & 14 & 15.70 \\
\hline 06Z151B & 0.292 & - & 2.0 & 0.13 & $>10000$ & $<10$ & 220 & $<0.5$ & 3 & 4.06 & 1.5 & 4 & 34 & 107 & - & 4.19 & $<10$ & 4 & 6.40 \\
\hline 06Z153A & 0.032 & - & 0.4 & 0.71 & 1045 & $<10$ & 30 & $<0.5$ & $<2$ & 0.90 & $<0.5$ & 12 & 37 & 91 & - & 2.86 & $<10$ & 1 & 2.59 \\
\hline 06Z156A & 0.112 & - & 5.7 & 0.07 & 180 & $<10$ & 30 & $<0.5$ & $<2$ & $>25.0$ & $<0.5$ & 1 & 3 & 11 & - & 0.65 & $<10$ & $>10000$ & $>100$ \\
\hline 06Z161A & $<0.005$ & - & $<0.2$ & 0.82 & 23 & $<10$ & 30 & $<0.5$ & $<2$ & 1.74 & $<0.5$ & 18 & 23 & 38 & - & 2.82 & $<10$ & 399 & $>100$ \\
\hline 06Z174A & $<0.005$ & - & 0.2 & 0.05 & 13 & $<10$ & 10 & $<0.5$ & $<2$ & $>25.0$ & $<0.5$ & 2 & 2 & 4 & - & 0.31 & $<10$ & 43 & 23.40 \\
\hline 06Z179A & $<0.005$ & - & $<0.2$ & 0.20 & 19 & $<10$ & 10 & $<0.5$ & $<2$ & 1.42 & $<0.5$ & 7 & 36 & 10 & - & 1.20 & $<10$ & 19 & 6.00 \\
\hline 06Z196B & $<0.005$ & - & 0.3 & 1.48 & 4 & $<10$ & 10 & $<0.5$ & $<2$ & 0.29 & $<0.5$ & 13 & 78 & 195 & - & 2.91 & $<10$ & 9 & 2.30 \\
\hline $06 Z 215 A$ & 0.012 & - & 6.5 & 0.94 & 203 & $<10$ & 200 & $<0.5$ & $<2$ & 0.01 & $<0.5$ & 5 & 20 & 162 & - & 9.04 & 10 & 6 & 1.60 \\
\hline 06Z217A & $<0.005$ & - & $<0.2$ & 0.11 & 4 & $<10$ & 20 & $<0.5$ & $<2$ & 0.02 & $<0.5$ & 1 & 17 & 6 & - & 0.68 & $<10$ & 4 & 0.10 \\
\hline 06Z231B & 0.013 & - & $<0.2$ & 0.19 & 19 & $<10$ & 20 & $<0.5$ & $<2$ & 16.30 & $<0.5$ & 2 & 5 & 3 & - & 1.61 & $<10$ & 5 & 0.10 \\
\hline 06Z244A & 0.021 & - & 0.6 & 2.40 & $<2$ & $<10$ & 40 & $<0.5$ & $<2$ & 0.79 & $<0.5$ & 21 & 84 & 725 & - & 4.37 & 10 & 3 & 0.10 \\
\hline 06Z282A & $<0.005$ & - & 0.4 & 0.39 & 28 & $<10$ & 160 & $<0.5$ & $<2$ & 0.31 & $<0.5$ & 7 & 10 & 50 & - & 3.52 & $<10$ & 3 & 0.10 \\
\hline 06Z285B & $<0.005$ & - & $<0.2$ & 0.34 & 3 & $<10$ & 30 & $<0.5$ & $<2$ & 5.70 & $<0.5$ & 3 & 9 & 8 & - & 3.10 & $<10$ & 2 & 0.03 \\
\hline 06Z307A & $<0.005$ & - & 0.2 & 0.32 & 10 & $<10$ & 70 & $<0.5$ & $<2$ & 0.03 & $<0.5$ & 4 & 12 & 41 & - & 1.77 & $<10$ & 2 & 0.29 \\
\hline 06Z336A & $<0.005$ & - & $<0.2$ & 0.07 & $<2$ & $<10$ & 20 & $<0.5$ & $<2$ & $>25.0$ & $<0.5$ & $<1$ & 2 & 1 & - & 0.13 & $<10$ & 2 & 0.03 \\
\hline 06Z342A & 0.008 & - & 0.2 & 1.29 & 10 & $<10$ & 50 & $<0.5$ & $<2$ & 0.42 & $<0.5$ & 13 & 36 & 71 & - & 3.49 & $<10$ & 2 & 1.19 \\
\hline 06Z345C & $<0.005$ & - & $<0.2$ & 1.42 & 2 & $<10$ & 20 & $<0.5$ & $<2$ & 5.36 & $<0.5$ & 8 & 24 & 21 & - & 3.24 & $<10$ & 1 & 0.08 \\
\hline 06Z355B & $<0.005$ & - & $<0.2$ & 0.03 & $<2$ & $<10$ & 10 & $<0.5$ & $<2$ & $>25.0$ & $<0.5$ & 1 & 1 & 1 & - & 0.66 & $<10$ & $<1$ & 0.09 \\
\hline 06Z375A & $<0.005$ & - & $<0.2$ & 0.69 & 3 & $<10$ & 20 & $<0.5$ & $<2$ & 3.42 & $<0.5$ & 5 & 14 & 5 & - & 4.49 & $<10$ & 1 & 0.04 \\
\hline 06Z387A & $<0.005$ & - & 0.3 & 0.40 & 26 & $<10$ & 170 & $<0.5$ & $<2$ & 0.08 & $<0.5$ & 2 & 14 & 33 & - & 2.46 & $<10$ & 1 & 0.07 \\
\hline 06Z388C & $<0.005$ & - & 0.7 & 1.80 & 54 & $<10$ & 130 & 1.1 & 4 & 0.40 & 12.4 & 49 & 19 & 460 & - & 34.50 & 10 & 1 & 0.16 \\
\hline 06Z394C & $<0.005$ & - & $<0.2$ & 0.23 & 26 & $<10$ & 40 & $<0.5$ & $<2$ & 0.02 & $<0.5$ & 2 & 12 & 26 & - & 2.50 & $<10$ & $<1$ & 0.03 \\
\hline 06Z404A & $<0.005$ & - & $<0.2$ & 0.25 & 5 & $<10$ & 160 & $<0.5$ & $<2$ & $>25.0$ & $<0.5$ & 5 & 3 & 19 & - & 1.37 & $<10$ & $<1$ & 0.05 \\
\hline 06Z412B & 0.008 & - & 0.3 & 1.48 & 22 & $<10$ & 110 & $<0.5$ & $<2$ & 0.07 & 4.3 & 15 & 14 & 469 & - & 12.00 & $<10$ & $<1$ & 0.08 \\
\hline 06Z420A & $<0.005$ & - & $<0.2$ & 1.13 & 430 & $<10$ & 70 & $<0.5$ & $<2$ & 12.25 & $<0.5$ & 37 & 334 & 14 & - & 4.52 & $<10$ & $<1$ & 0.02 \\
\hline 06Z430A & $<0.005$ & - & 0.2 & 0.79 & 8 & $<10$ & 40 & $<0.5$ & $<2$ & 6.02 & $<0.5$ & 7 & 14 & 105 & - & 3.08 & $<10$ & $<1$ & 0.18 \\
\hline 06Z444A & $<0.005$ & - & $<0.2$ & 1.27 & 15 & $<10$ & 50 & $<0.5$ & $<2$ & 3.26 & $<0.5$ & 11 & 30 & 18 & - & 3.24 & $<10$ & $<1$ & 0.03 \\
\hline 06Z489B & $<0.005$ & - & $<0.2$ & 0.26 & 5 & $<10$ & 20 & $<0.5$ & $<2$ & 20.60 & $<0.5$ & 2 & 4 & 19 & - & 0.86 & $<10$ & $<1$ & 0.07 \\
\hline 06Z490A & 0.006 & - & 0.2 & 0.17 & 417 & $<10$ & 20 & $<0.5$ & $<2$ & 0.18 & 0.6 & 1 & 12 & 4 & - & 3.46 & $<10$ & 9 & 8.90 \\
\hline 06Z492A & 7.82 & - & 2.0 & 0.23 & $>10000$ & $<10$ & 10 & $<0.5$ & 17 & 1.81 & $<0.5$ & 33 & 3 & 5 & - & 29.00 & $<10$ & $<1$ & 0.03 \\
\hline
\end{tabular}


Table 2. (continued)

\begin{tabular}{|c|c|c|c|c|c|c|c|c|c|c|c|c|c|c|c|c|c|c|c|}
\hline Sample Number & $\begin{array}{l}\mathrm{K} \\
\% \\
\end{array}$ & $\begin{array}{c}\mathrm{La} \\
\mathrm{ppm}\end{array}$ & $\begin{array}{c}\mathrm{Mg} \\
\%\end{array}$ & $\begin{array}{c}\mathrm{Mn} \\
\mathrm{ppm}\end{array}$ & $\begin{array}{c}\text { Mo } \\
\mathrm{ppm}\end{array}$ & $\begin{array}{c}\mathrm{Na} \\
\% \\
\end{array}$ & $\begin{array}{c}\mathrm{Ni} \\
\mathrm{ppm}\end{array}$ & $\begin{array}{l}\mathrm{P} \\
\% \\
\end{array}$ & $\begin{array}{c}\mathrm{Pb} \\
\mathrm{ppm}\end{array}$ & $\begin{array}{r}\mathrm{S} \\
\% \\
\end{array}$ & $\begin{array}{c}\text { Sb } \\
\mathrm{ppm}\end{array}$ & $\begin{array}{c}\mathrm{Sc} \\
\mathrm{ppm}\end{array}$ & $\begin{array}{c}\mathrm{Sr} \\
\mathrm{ppm}\end{array}$ & $\begin{array}{l}\mathrm{Ti} \\
\% \\
\end{array}$ & $\begin{array}{c}\mathrm{Tl} \\
\mathrm{ppm}\end{array}$ & $\begin{array}{c}\text { U } \\
\text { ppm }\end{array}$ & $\begin{array}{c}\text { V } \\
\text { ppm }\end{array}$ & $\begin{array}{c}\text { W } \\
\mathrm{ppm}\end{array}$ & $\begin{array}{c}\mathrm{Zn} \\
\mathrm{ppm}\end{array}$ \\
\hline 06Z108A & 0.06 & 20 & 1.04 & 210 & 1 & 0.02 & 40 & 400 & 5 & 0.60 & 2 & 3 & 87 & $<0.01$ & $<10$ & $<10$ & 19 & $<10$ & 23 \\
\hline 06Z125A & .01 & $<10$ & 0.40 & 122 & 3 & $<0.01$ & 21 & 250 & 4 & $<0.01$ & 6 & 1 & 245 & 0.01 & 10 & 10 & 43 & $<10$ & 38 \\
\hline 06Z143B & 06 & 10 & 0.18 & 412 & $<1$ & $<0.01$ & 22 & 220 & 2 & 0.01 & $<2$ & 1 & 137 & $<0.01$ & $<10$ & $<10$ & 2 & $<10$ & \\
\hline 06Z151A & 05 & 10 & 0.02 & 249 & 18 & 0.01 & 68 & 800 & 1450 & 0.06 & 629 & $\Gamma$ & 89 & $<0.01$ & $<10$ & 20 & 48 & 50 & 260 \\
\hline 06Z151B & 04 & 10 & 0.03 & 157 & 4 & $<0.01$ & 22 & 260 & 166 & 0.02 & 132 & 2 & 141 & $<0.01$ & $<10$ & $<10$ & 22 & $<10$ & 56 \\
\hline 06Z153A & 12 & 20 & 0.29 & 99 & 2 & 0.02 & 33 & 560 & 74 & $<0.01$ & 75 & 2 & 7 & $<0.01$ & $<10$ & $<10$ & 15 & $<10$ & 97 \\
\hline 06Z156A & 01 & $<10$ & 0.24 & 359 & $<1$ & $<0.01$ & 7 & 50 & 12 & 1.00 & 91 & 3 & 12 & $<0.01$ & $<10$ & 10 & 6 & 10 & 37 \\
\hline 06Z161A & 17 & 10 & 0.91 & 269 & 1 & $<0.01$ & 43 & 450 & 11 & 1.17 & 2 & 2 & 58 & $<0.01$ & $<10$ & $<10$ & 12 & $<10$ & 46 \\
\hline 06Z174A & 0.02 & $<10$ & 0.64 & 132 & $<1$ & 0.01 & 4 & 110 & 4 & $<0.01$ & 3 & 1 & 1205 & $<0.01$ & $<10$ & 10 & 2 & $<10$ & 6 \\
\hline 06Z179A & .07 & $<10$ & 0.13 & 447 & 5 & $<0.01$ & 12 & 260 & 3 & 0.41 & $<2$ & 1 & 27 & $<0.01$ & $<10$ & $<10$ & 5 & $<10$ & \\
\hline 06Z196B & .02 & 10 & 1.29 & 520 & $<1$ & 0.04 & 47 & 540 & 6 & 0.01 & $<2$ & 4 & 12 & 0.05 & $<10$ & $<10$ & 43 & $<10$ & 56 \\
\hline 06Z215A & 0.40 & 10 & 0.09 & 79 & 1 & 0.06 & 16 & 520 & 2000 & 0.87 & 117 & 4 & 15 & $<0.01$ & $<10$ & $<10$ & 35 & $<10$ & 572 \\
\hline 06Z217A & 0.03 & $<10$ & 0.01 & 50 & $<1$ & 0.01 & 3 & 40 & 14 & 0.01 & $<2$ & $<1$ & 1 & $<0.01$ & $<10$ & $<10$ & 2 & $<10$ & 5 \\
\hline 06Z231B & 0.08 & $<10$ & 0.12 & 333 & $<1$ & 0.02 & 9 & 210 & 4 & 0.03 & 4 & 2 & 195 & $<0.01$ & $<10$ & $<10$ & 4 & $<10$ & 23 \\
\hline 06Z244A & 0.06 & 10 & 2.53 & 831 & 2 & 0.12 & 45 & 570 & 5 & 0.09 & $<2$ & 7 & 53 & 0.10 & $<10$ & $<10$ & 88 & $<10$ & 57 \\
\hline $06 Z 282 A$ & 0.07 & $<10$ & 0.03 & 110 & 19 & 0.01 & 79 & 390 & 21 & 0.02 & 7 & 2 & 11 & $<0.01$ & $<10$ & $<10$ & 32 & $<10$ & 131 \\
\hline 06Z285B & 06 & $<10$ & 0.86 & 800 & 2 & 0.02 & 12 & 210 & 8 & 0.01 & $<2$ & 2 & 113 & $<0.01$ & $<10$ & $<10$ & 5 & $<10$ & 23 \\
\hline 06Z307A & 0.06 & $<10$ & 0.02 & 53 & 13 & 0.01 & 40 & 180 & 14 & 0.02 & 6 & 2 & 9 & $<0.01$ & $<10$ & $<10$ & 65 & $<10$ & 141 \\
\hline BZ336A & 0.01 & $<10$ & 0.45 & 105 & $<1$ & 0.02 & $<1$ & 60 & 2 & 0.01 & $<2$ & $<1$ & 360 & $<0.01$ & $<10$ & $<10$ & $<1$ & $<10$ & $\Sigma$ \\
\hline 06Z342A & 0.17 & 10 & 0.59 & 275 & 1 & 0.07 & 38 & 590 & 21 & 0.67 & 6 & 4 & 13 & $<0.01$ & $<10$ & $<10$ & 39 & $<10$ & 42 \\
\hline $6 Z 345 C$ & 0.25 & 30 & 1.16 & 1380 & $<1$ & 0.04 & 26 & 720 & 20 & 0.12 & 2 & 4 & 293 & $<0.01$ & $<10$ & $<10$ & 19 & $<10$ & 39 \\
\hline DZ355B & 0.01 & $<10$ & 8.97 & 651 & $<1$ & 0.02 & $<1$ & 30 & 2 & $<0.01$ & 2 & $<1$ & 200 & $<0.01$ & $<10$ & $<10$ & 3 & $<10$ & \\
\hline $06 Z 375 A$ & 0.19 & 10 & 0.63 & 1650 & 1 & 0.03 & 19 & 300 & 10 & 0.02 & $<2$ & 2 & 63 & $<0.01$ & $<10$ & $<10$ & 7 & $<10$ & 15 \\
\hline 06Z387A & 0.09 & $<10$ & 0.03 & 94 & 22 & 0.02 & 25 & 310 & 8 & 0.02 & 3 & . & 19 & $<0.01$ & $<10$ & $<10$ & 47 & $<10$ & 76 \\
\hline $6 Z 388 C$ & 12 & 10 & 0.13 & 460 & 52 & 0.01 & 578 & 2700 & {$[9$} & 0.12 & 6 & 3 & 8 & $<0.01$ & $<10$ & 40 & 114 & $<10$ & 2560 \\
\hline 06Z394C & 0.02 & $<10$ & 0.01 & 70 & 7 & 0. & 30 & 240 & 16 & 0.01 & $<2$ & 3 & 5 & $<0.01$ & $<10$ & $<10$ & 10 & $<10$ & 46 \\
\hline 06Z404A & 07 & $<$ & 1.24 & 262 & 2 & 0.02 & 19 & 210 & 9 & $<0.01$ & 2 & 2 & 703 & $<0.01$ & $<10$ & $<10$ & 5 & $<10$ & 79 \\
\hline 06Z412B & 10 & $<$ & 02 & 182 & 38 & 0. & 220 & 100 & 0 & 0.09 & 15 & & 10 & $<0.01$ & $<10$ & 20 & 65 & $<10$ & 754 \\
\hline 06Z420A & & $<10$ & .40 & 1580 & $<1$ & 0.02 & 274 & 640 & 16 & 0.09 & 2 & & 278 & $<0.01$ & $<10$ & $<10$ & 25 & $<10$ & 77 \\
\hline 06Z430A & 0.2 & 10 & 53 & 620 & 4 & 0. & 38 & 410 & 8 & 0.14 & $<2$ & & 186 & $<0.01$ & $<10$ & $<10$ & 15 & $<10$ & 88 \\
\hline 06Z444A & 0.25 & 20 & 66 & 530 & $<1$ & 0. & 37 & 470 & 7 & 0.09 & 2 & & 66 & $<0.01$ & $<10$ & $<10$ & 18 & $<10$ & 61 \\
\hline 06Z489B & 07 & $<10$ & .50 & 227 & 1 & 0.02 & 9 & 80 & 16 & $<0.01$ & $<2$ & 1 & 413 & $<0.01$ & $<10$ & $<10$ & 9 & $<10$ & 40 \\
\hline 06Z490A & 0.06 & $<10$ & 0.04 & 56 & 10 & 0.01 & 3 & 30 & 5 & 0.02 & 501 & $<1$ & 9 & $<0.01$ & $<10$ & $<10$ & 8 & $<10$ & 86 \\
\hline $6 Z 492 A$ & 0.06 & $<10$ & .04 & 49 & 23 & 0.03 & 17 & 90 & 94 & 10.0 & 02 & 2 & 36 & $<0.01$ & 10 & 10 & 6 & 30 & \\
\hline
\end{tabular}


Table 2. (continued).

\begin{tabular}{|c|c|c|c|c|c|c|c|c|c|c|c|c|c|c|c|c|c|c|c|}
\hline Sample Number & $\begin{array}{c}\mathrm{Au} \\
\mathrm{ppm}\end{array}$ & $\begin{array}{c}\mathrm{Au}(+) \\
\mathrm{ppm}\end{array}$ & $\begin{array}{c}\mathrm{Ag} \\
\mathrm{ppm}\end{array}$ & $\begin{array}{l}\mathrm{Al} \\
\% \\
\end{array}$ & $\begin{array}{c}\text { As } \\
\text { ppm }\end{array}$ & $\begin{array}{c}\text { B } \\
\text { ppm }\end{array}$ & $\begin{array}{c}\mathrm{Ba} \\
\mathrm{ppm}\end{array}$ & $\begin{array}{c}\mathrm{Be} \\
\mathrm{ppm}\end{array}$ & $\begin{array}{c}\mathrm{Bi} \\
\text { ppm }\end{array}$ & $\begin{array}{l}\mathrm{Ca} \\
\% \\
\end{array}$ & $\begin{array}{c}\text { Cd } \\
\text { ppm }\end{array}$ & $\begin{array}{c}\text { Co } \\
\text { ppm }\end{array}$ & $\begin{array}{c}\mathrm{Cr} \\
\mathrm{ppm}\end{array}$ & $\begin{array}{c}\mathrm{Cu} \\
\mathrm{ppm}\end{array}$ & $\begin{array}{c}\mathrm{Cu}(+) \\
\%\end{array}$ & $\begin{array}{l}\mathrm{Fe} \\
\% \\
\end{array}$ & $\begin{array}{c}\mathrm{Ga} \\
\mathrm{ppm}\end{array}$ & $\begin{array}{c}\mathrm{Hg} \\
\mathrm{ppm}\end{array}$ & \begin{tabular}{|c}
$\mathrm{Hg}(+)$ \\
$\mathrm{ppm}$ \\
\end{tabular} \\
\hline 06Z492D & 2.64 & - & 0.3 & 0.38 & $>10000$ & $<10$ & 10 & $<0.5$ & $<2$ & 6.63 & $<0.5$ & 3 & 11 & 5 & - & 3.09 & $<10$ & $<1$ & 0.58 \\
\hline 06Z523B & 0.099 & - & 0.5 & 0.25 & 3710 & $<10$ & 110 & $<0.5$ & $<2$ & 0.06 & $<0.5$ & 2 & 14 & 11 & - & 1.41 & $<10$ & $<1$ & 0.07 \\
\hline 06MBW48A & 1.51 & - & 0.3 & 0.45 & $>10000$ & $<10$ & 20 & $<0.5$ & $<2$ & 0.26 & $<0.5$ & 33 & 8 & 5 & - & 5.80 & $<10$ & $<1$ & 0.10 \\
\hline 06MBW50A & 0.015 & - & $<0.2$ & 0.53 & 428 & $<10$ & 10 & $<0.5$ & $<2$ & 0.45 & $<0.5$ & 4 & 23 & 25 & - & 1.28 & $<10$ & $<1$ & 0.02 \\
\hline 06MBW73A & 0.132 & - & 0.4 & 0.26 & 1760 & $<10$ & 100 & $<0.5$ & $<2$ & 0.39 & $<0.5$ & 5 & 12 & 26 & - & 3.52 & $<10$ & $<1$ & 0.06 \\
\hline 06MBW81A & 0.022 & - & $<0.2$ & 1.53 & 723 & $<10$ & 50 & $<0.5$ & $<2$ & 0.22 & $<0.5$ & 10 & 24 & 13 & - & 2.90 & $<10$ & $<1$ & 0.04 \\
\hline 06MBW82C & 0.017 & - & $<0.2$ & 0.09 & 277 & $<10$ & 10 & $<0.5$ & $<2$ & 0.68 & $<0.5$ & 3 & 11 & 2 & - & 0.90 & $<10$ & $<1$ & 0.02 \\
\hline 06MBW83C & 0.191 & - & 0.8 & 0.06 & 1030 & $<10$ & 10 & $<0.5$ & $<2$ & 19.30 & 0.8 & 1 & 2 & 45 & - & 0.78 & $<10$ & $<1$ & 0.34 \\
\hline 06MBW86A & 0.040 & - & 13.9 & 0.09 & 152 & $<10$ & $<10$ & $<0.5$ & $<2$ & 0.17 & $<0.5$ & 5 & 16 & 3300 & - & 1.09 & $<10$ & 7 & 7.91 \\
\hline 06MBW90B & $<0.005$ & - & 0.7 & 0.11 & 59 & $<10$ & 10 & $<0.5$ & $<2$ & 2.15 & $<0.5$ & 8 & 14 & 210 & - & 1.25 & $<10$ & $<1$ & 0.51 \\
\hline 06MBW90C & $<0.005$ & - & $<0.2$ & 0.17 & 80 & $<10$ & 10 & $<0.5$ & $<2$ & $>25.0$ & $<0.5$ & 4 & 4 & 12 & - & 1.03 & $<10$ & $<1$ & 0.16 \\
\hline 06MBW92A & $<0.005$ & - & $<0.2$ & 0.06 & 8 & $<10$ & $<10$ & $<0.5$ & $<2$ & 0.89 & $<0.5$ & 1 & 10 & 20 & - & 0.76 & $<10$ & $<1$ & 0.08 \\
\hline 06MBW107A & 0.005 & - & 0.3 & 0.10 & 24 & $<10$ & 10 & $<0.5$ & $<2$ & 1.52 & $<0.5$ & 2 & 9 & 654 & - & 0.83 & $<10$ & $<1$ & 0.03 \\
\hline 06MBW161A & $<0.005$ & - & $<0.2$ & 1.01 & 6 & $<10$ & 40 & $<0.5$ & $<2$ & 9.84 & $<0.5$ & 19 & 37 & 44 & - & 2.76 & $<10$ & 1 & 0.04 \\
\hline 06MBW163A & $<0.005$ & - & $<0.2$ & 0.02 & 3 & $<10$ & $<10$ & $<0.5$ & $<2$ & 1.14 & $<0.5$ & $<1$ & 15 & 2 & - & 0.53 & $<10$ & $<1$ & 0.03 \\
\hline 06MBW168A & $<0.005$ & - & $<0.2$ & 0.77 & $<2$ & $<10$ & 10 & $<0.5$ & $<2$ & 9.34 & $<0.5$ & 2 & 13 & 6 & - & 0.58 & $<10$ & $<1$ & 0.02 \\
\hline 06MBW179A & $<0.005$ & - & $<0.2$ & 1.10 & $<2$ & $<10$ & 10 & $<0.5$ & $<2$ & 0.34 & $<0.5$ & 9 & 74 & 11 & - & 2.07 & $<10$ & $<1$ & 0.03 \\
\hline 06MBW189A & $<0.005$ & - & $<0.2$ & 0.03 & 4 & $<10$ & 90 & $<0.5$ & $<2$ & $>25.0$ & 0.7 & 1 & 1 & 1 & - & 1.14 & $<10$ & $<1$ & 0.02 \\
\hline 06MBW232A & $<0.005$ & - & $<0.2$ & 1.72 & 3 & $<10$ & 30 & $<0.5$ & $<2$ & 1.12 & $<0.5$ & 5 & 15 & 4 & - & 0.93 & $<10$ & $<1$ & 0.01 \\
\hline 06MBW262A & 0.005 & - & 0.2 & 0.78 & 6 & $<10$ & 480 & $<0.5$ & $<2$ & 0.21 & $<0.5$ & 2 & 15 & 27 & - & 1.36 & $<10$ & $<1$ & 0.04 \\
\hline 06MBW263B & $<0.005$ & - & 0.6 & 1.10 & 5 & $<10$ & 90 & $<0.5$ & $<2$ & 0.05 & 0.5 & 6 & 16 & 76 & - & 2.79 & $<10$ & $<1$ & 0.23 \\
\hline 06MBW264B & $<0.005$ & - & $<0.2$ & 0.02 & $<2$ & $<10$ & 10 & $<0.5$ & $<2$ & 0.02 & $<0.5$ & $<1$ & 14 & 8 & - & 0.65 & $<10$ & $<1$ & 0.02 \\
\hline 06MBW266B & $<0.005$ & - & $<0.2$ & 0.76 & 41 & $<10$ & 10 & $<0.5$ & $<2$ & 0.32 & $<0.5$ & 2 & 24 & 7 & - & 0.93 & $<10$ & $<1$ & 0.03 \\
\hline 06MBW273A & $>10.0$ & 22 & 12.4 & 0.12 & $>10000$ & $<10$ & 100 & $<0.5$ & 35 & 0.45 & 1.7 & 7 & 1 & 271 & - & 21.10 & $<10$ & 5 & 2.60 \\
\hline 06MBW277A & 0.133 & - & 0.4 & 0.20 & 711 & $<10$ & 10 & $<0.5$ & $<2$ & $>25.0$ & 0.8 & 3 & 3 & 19 & - & 0.51 & $<10$ & $<1$ & 0.20 \\
\hline 06MBW287A & 0.017 & - & 0.3 & 1.11 & 286 & $<10$ & 50 & 1.2 & $<2$ & 0.25 & 0.5 & 17 & 42 & 28 & - & 11.45 & $<10$ & $<1$ & 0.07 \\
\hline 06MBW289A & $<0.005$ & - & $<0.2$ & 0.43 & 124 & 10 & 10 & $<0.5$ & $<2$ & 0.34 & $<0.5$ & 2 & 12 & 8 & - & 1.00 & $<10$ & $<1$ & 0.02 \\
\hline 06MBW309A & 0.005 & - & $<0.2$ & 0.16 & 47 & $<10$ & 10 & $<0.5$ & $<2$ & 0.04 & $<0.5$ & 2 & 16 & 2 & - & 0.86 & $<10$ & $<1$ & 0.08 \\
\hline 06MBW330A & 0.011 & - & $<0.2$ & 0.05 & 51 & $<10$ & 20 & $<0.5$ & $<2$ & $>25.0$ & $<0.5$ & 1 & 3 & 6 & - & 1.99 & $<10$ & 14 & 13.60 \\
\hline 06MBW336A & $<0.005$ & - & $<0.2$ & 1.92 & 6 & $<10$ & 20 & $<0.5$ & $<2$ & 0.67 & $<0.5$ & 13 & 69 & 23 & - & 2.98 & 10 & $<1$ & 0.07 \\
\hline 06MBW403A & $<0.005$ & - & 0.5 & 0.67 & 17 & $<10$ & 250 & $<0.5$ & $<2$ & 0.07 & 4.5 & 7 & 13 & 33 & - & 1.95 & $<10$ & $<1$ & 0.04 \\
\hline 06MBW438A & $<0.005$ & - & $<0.2$ & 0.11 & 12 & $<10$ & 40 & $<0.5$ & $<2$ & $>25.0$ & $<0.5$ & 2 & 2 & $<1$ & - & 0.67 & $<10$ & $<1$ & 0.04 \\
\hline 06MBW454A & $<0.005$ & - & 0.4 & 0.96 & 34 & $<10$ & 280 & $<0.5$ & $<2$ & 0.13 & 1.9 & 6 & 17 & 93 & - & 6.51 & $<10$ & $<1$ & 0.08 \\
\hline 06MBW465A & $<0.005$ & - & $<0.2$ & 0.05 & 5 & $<10$ & 10 & $<0.5$ & $<2$ & 9.54 & $<0.5$ & $<1$ & 4 & 2 & - & 0.39 & $<10$ & $<1$ & 0.02 \\
\hline
\end{tabular}


Table 2. (continued)

\begin{tabular}{|c|c|c|c|c|c|c|c|c|c|c|c|c|c|c|c|c|c|c|c|}
\hline Sample Number & $\begin{array}{l}\mathrm{K} \\
\% \\
\end{array}$ & $\begin{array}{c}\mathrm{La} \\
\mathrm{ppm}\end{array}$ & $\begin{array}{c}\mathrm{Mg} \\
\% \\
\end{array}$ & $\begin{array}{c}\mathrm{Mn} \\
\mathrm{ppm}\end{array}$ & $\begin{array}{c}\text { Mo } \\
\text { ppm }\end{array}$ & $\begin{array}{c}\mathrm{Na} \\
\% \\
\end{array}$ & $\begin{array}{c}\mathrm{Ni} \\
\mathrm{ppm}\end{array}$ & $\begin{array}{l}\mathrm{P} \\
\% \\
\end{array}$ & $\begin{array}{c}\mathrm{Pb} \\
\mathrm{ppm}\end{array}$ & $\begin{array}{l}\mathrm{S} \\
\% \\
\end{array}$ & $\begin{array}{c}\text { Sb } \\
\text { ppm }\end{array}$ & $\begin{array}{c}\text { Sc } \\
\text { ppm }\end{array}$ & $\begin{array}{c}\mathrm{Sr} \\
\mathrm{ppm}\end{array}$ & $\begin{array}{l}\mathrm{Ti} \\
\% \\
\end{array}$ & $\begin{array}{c}\text { TI } \\
\text { ppm }\end{array}$ & $\begin{array}{c}\text { U } \\
\text { ppm }\end{array}$ & $\begin{array}{c}\text { V } \\
\text { ppm }\end{array}$ & $\begin{array}{c}\text { W } \\
\mathrm{ppm}\end{array}$ & $\begin{array}{c}\mathrm{Zn} \\
\mathrm{ppm}\end{array}$ \\
\hline 06Z492D & 0.08 & $=10$ & 0.06 & 309 & 5 & 0.06 & 10 & 70 & 21 & 0.45 & 94 & 3 & 105 & $<0.01$ & $<10$ & $<10$ & 9 & 1310 & 29 \\
\hline 06Z523B & 0.06 & 10 & .02 & 49 & 6 & 0.01 & 10 & 110 & 12 & 0.16 & 20 & 1 & 4 & 0.01 & $<10$ & $<10$ & 23 & $<10$ & 33 \\
\hline 06MBW48A & 0.05 & 10 & 11 & 128 & 1 & 0.02 & 41 & 20 & 7 & 4.33 & 136 & & 15 & $<0.01$ & $<10$ & $<10$ & & $<10$ & 11 \\
\hline 06MBW50A & 0.02 & $<10$ & .38 & 174 & $<1$ & 0.03 & 9 & 180 & 5 & 0.04 & $<2$ & 2 & 13 & 0.02 & $<10$ & $<10$ & 15 & $<10$ & 17 \\
\hline 06MBW79A & 0.04 & $<10$ & .06 & 44 & 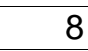 & 0.01 & 15 & 2090 & 14 & .35 & 19 & & 69 & $<0.01$ & $<10$ & $<10$ & 17 & $<10$ & 98 \\
\hline 06MBW81A & 0.19 & 10 & .72 & 457 & 1 & 0.03 & 35 & 410 & 7 & 0.04 & $<2$ & 2 & 11 & 0.01 & $<10$ & $<10$ & 21 & $<10$ & 69 \\
\hline 06MBW82C & 0.02 & $<10$ & 0.01 & 61 & 4 & 0.01 & 5 & 10 & 3 & 0.06 & 4 & $<1$ & 5 & $<0.01$ & $<10$ & $<10$ & & $<10$ & \\
\hline 06MBW83C & 0.02 & $<10$ & 0.09 & 265 & $<1$ & 0.01 & 2 & 10 & 8 & $<0.01$ & 1585 & $<1$ & 192 & 0.01 & $<10$ & $<10$ & $<1$ & $<10$ & 101 \\
\hline 06MBW86A & 0.01 & $<10$ & 0.01 & 52 & & 0.0 & 9 & 10 & $<2$ & 0.02 & 322 & $<1$ & 2 & 0.01 & $<10$ & $<10$ & & $<10$ & 29 \\
\hline 06MBW90B & 0.02 & $<10$ & 0.13 & 89 & 1 & 0.01 & 9 & 30 & 5 & 0.01 & 20 & $<1$ & 6 & $<0.01$ & $<10$ & $<10$ & & $<10$ & 25 \\
\hline 06MBW90C & 0.03 & $<10$ & 7.54 & 572 & 1 & 0.02 & 8 & 80 & 11 & $<0.01$ & 13 & 2 & 88 & $<0.01$ & $<10$ & $<10$ & 16 & $<10$ & 58 \\
\hline 06MBW92A & 0.02 & $<10$ & 0.04 & 76 & 1 & 0.01 & 2 & 10 & $<2$ & 0.01 & 0 & $<1$ & 2 & $<0.01$ & $<10$ & $<10$ & & $<10$ & 4 \\
\hline 06MBW107A & 0.02 & $<10$ & 0.30 & 87 & 1 & 0.01 & 2 & 10 & 3 & 0.02 & 11 & $<1$ & 5 & $<0.01$ & $<10$ & $<10$ & & $<10$ & 7 \\
\hline 06MBW161A & 0.06 & $<10$ & 0.90 & 642 & $<1$ & 0.03 & 48 & 160 & 3 & 0.02 & $<2$ & 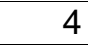 & 439 & 0.04 & $<10$ & $<10$ & 30 & $<10$ & 45 \\
\hline 06MBW163A & $<0.01$ & $<10$ & 0.02 & 48 & 1 & 0.01 & 1 & 10 & 4 & 0.01 & $<2$ & $<1$ & 43 & $<0.01$ & $<10$ & $<10$ & $<1$ & $<10$ & 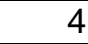 \\
\hline 06MBW168A & 0.05 & $<10$ & 0.40 & 51 & $<1$ & 0.0 & 5 & 60 & 3 & 0.01 & 2 & 2 & 410 & $<0.01$ & $<10$ & $<10$ & 13 & $<10$ & 10 \\
\hline $79 A$ & 0.02 & 10 & 87 & 573 & 1 & 0.1 & 32 & 700 & 5 & 0.01 & $<2$ & 5 & 12 & 0.01 & $<10$ & $<10$ & 29 & $<10$ & 33 \\
\hline 06MBW189A & 0.01 & $<10$ & 11 & 151 & $<1$ & 0.02 & 2 & 10 & 2 & $<0.01$ & $<2$ & 1 & 367 & $<0.01$ & 10 & $<10$ & $<1$ & $<10$ & 32 \\
\hline MBW232A & 16 & 10 & 0.21 & 528 & $<1$ & 0.11 & 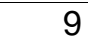 & 740 & 7 & 0.01 & $<2$ & & 70 & 0.03 & 10 & $<10$ & 25 & $<10$ & 0 \\
\hline MBW262A & 0.24 & 1 & 14 & 36 & 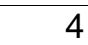 & 0.02 & 22 & 1200 & 6 & 0.01 & $<2$ & & 40 & $<0.01$ & -10 & $<10$ & 44 & $<10$ & 68 \\
\hline MBW263B & 0.09 & 10 & 64 & 50 & 11 & 0.02 & 70 & 180 & 11 & 0.02 & $<2$ & 2 & 15 & $<0.01$ & K10 & $<10$ & 61 & $<10$ & 171 \\
\hline MBW264B & 0.01 & $<10$ & $<0.01$ & 0 & 1 & 0.01 & 4 & 30 & 2 & .01 & $<2$ & $<1$ & 2 & $<0.01$ & $<10$ & $<10$ & 1 & $<10$ & 8 \\
\hline 06MBW266B & 0.01 & $<10$ & 0.80 & 8 & 1 & 0.03 & 15 & 370 & $<2$ & 0.01 & $<2$ & 2 & 19 & $<0.01$ & $<10$ & $<10$ & 34 & $<10$ & 17 \\
\hline MBW273A & 0.05 & $<10$ & 0.01 & 2 & 1 & 0.02 & 18 & 90 & 2600 & 3.42 & 650 & $<1$ & 180 & $<0.01$ & $<10$ & $<10$ & 8 & 80 & 27 \\
\hline 06MBW277A & 0 & 10 & & 0 & $<1$ & 0.02 & 20 & 130 & 24 & $<0.01$ & 24 & & 527 & $<0.01$ & $<10$ & $<10$ & 12 & $<10$ & 45 \\
\hline 06MBW287A & 0 & 10 & & & 2 & 0.6 & 81 & 1030 & 14 & 0.01 & 3 & & 22 & 0.02 & $<10$ & $<10$ & 48 & $<10$ & 68 \\
\hline MBW289A & 03 & $<10$ & & 112 & 1 & 0. & 3 & 390 & 3 & 0.01 & 2 & & 19 & 0.20 & 10 & $<10$ & 25 & $<10$ & \\
\hline 06MBW309A & 01 & $<10$ & 0 & 14 & 2 & 0. & $\varepsilon$ & 20 & 2 & 0.01 & $<2$ & & 2 & $<0.01$ & $<10$ & $<10$ & 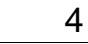 & $<10$ & \\
\hline 06MBW330A & & $<10$ & 99 & 832 & 2 & 0 & 3 & 70 & 4 & 0.01 & 80 & & 155 & $<0.01$ & $\leqslant 10$ & $<10$ & 13 & $<10$ & \\
\hline 06MBW336A & & 2 & & 9 & $<1$ & & 3 & 1230 & 4 & $<0.01$ & $<2$ & & 11 & 0.02 & $<10$ & $<10$ & 48 & $<10$ & 58 \\
\hline MBW403A & & 10 & & 2 & 7 & 0. & 58 & 220 & $s$ & 0.01 & $<2$ & & 10 & $<0.01$ & $<10$ & $<10$ & 27 & $<10$ & 132 \\
\hline MBW438A & 05 & $<10$ & 56 & 400 & $<$ & 0.02 & 2 & 100 & 3 & $<0.01$ & 4 & 1 & 215 & $<0.01$ & $<10$ & $<10$ & 1 & $<10$ & \\
\hline 06MBW454A & 0.12 & $<10$ & 10 & 60 & 32 & 0.01 & 69 & 600 & 16 & 0.04 & 4 & 2 & 15 & $<0.01$ & $<10$ & $<10$ & 108 & $<10$ & 247 \\
\hline $465 \mathrm{~A}$ & 0.01 & $<10$ & 3.74 & 186 & 1 & 01 & $<1$ & 40 & 11 & $<0.01$ & & <1 & 90 & $<0.01$ & 10 & 10 & & 10 & I. \\
\hline
\end{tabular}


Table 2. (continued).

\begin{tabular}{|c|c|c|c|c|c|c|c|c|c|c|c|c|c|c|c|c|c|c|c|}
\hline Sample Number & $\begin{array}{c}\mathrm{Au} \\
\mathrm{ppm}\end{array}$ & $\begin{array}{l}\mathrm{Au}(+) \\
\mathrm{ppm}\end{array}$ & $\begin{array}{c}\mathrm{Ag} \\
\mathrm{ppm}\end{array}$ & $\begin{array}{l}\mathrm{Al} \\
\% \\
\end{array}$ & $\begin{array}{c}\text { As } \\
\text { ppm }\end{array}$ & $\begin{array}{c}\mathrm{B} \\
\mathrm{ppm}\end{array}$ & $\begin{array}{c}\mathrm{Ba} \\
\mathrm{ppm}\end{array}$ & $\begin{array}{c}\mathrm{Be} \\
\mathrm{ppm}\end{array}$ & $\begin{array}{c}\mathrm{Bi} \\
\mathrm{ppm}\end{array}$ & $\begin{array}{l}\mathrm{Ca} \\
\% \\
\end{array}$ & $\begin{array}{c}\mathrm{Cd} \\
\mathrm{ppm}\end{array}$ & $\begin{array}{c}\text { Co } \\
\text { ppm }\end{array}$ & $\begin{array}{c}\mathrm{Cr} \\
\mathrm{ppm}\end{array}$ & $\begin{array}{c}\mathrm{Cu} \\
\mathrm{ppm}\end{array}$ & $\begin{array}{c}\mathrm{Cu}(+) \\
\% \\
\end{array}$ & $\begin{array}{l}\mathrm{Fe} \\
\% \\
\end{array}$ & $\begin{array}{c}\mathrm{Ga} \\
\mathrm{ppm}\end{array}$ & $\begin{array}{c}\mathrm{Hg} \\
\mathrm{ppm}\end{array}$ & $\begin{array}{c}\mathrm{Hg}(+) \\
\mathrm{ppm} \\
\end{array}$ \\
\hline 06MBW472A & 0.017 & - & $<0.2$ & 0.12 & 71 & $<10$ & 10 & $<0.5$ & $<2$ & 6.03 & $<0.5$ & 22 & 9 & 5 & - & 1.51 & $<10$ & $<1$ & 0.05 \\
\hline 06MBW484A & $<0.005$ & - & 0.5 & 0.44 & 13 & $<10$ & 1740 & $<0.5$ & $<2$ & 0.09 & $<0.5$ & 2 & 20 & 113 & - & 1.31 & $<10$ & $<1$ & 0.05 \\
\hline 06MBW565A & $<0.005$ & - & $<0.2$ & 0.06 & 6 & $<10$ & 30 & $<0.5$ & $<2$ & 8.16 & $<0.5$ & 1 & 12 & 7 & - & 0.49 & $<10$ & $<1$ & 0.03 \\
\hline 06MBW568A & $<0.005$ & - & 0.6 & 0.41 & 28 & $<10$ & 200 & $<0.5$ & $<2$ & 0.04 & $<0.5$ & 4 & 16 & 26 & - & 2.67 & $<10$ & $<1$ & 0.08 \\
\hline 06MBW572A & $<0.005$ & - & 0.2 & 1.48 & 13 & $<10$ & 340 & $<0.5$ & $<2$ & 0.06 & $<0.5$ & 10 & 20 & 28 & - & 1.66 & $<10$ & $<1$ & 0.03 \\
\hline 06MBW573A & $<0.005$ & - & 0.3 & 0.34 & 14 & $<10$ & 280 & $<0.5$ & $<2$ & 0.03 & $<0.5$ & 1 & 15 & 2 & - & 0.46 & $<10$ & $<1$ & 0.86 \\
\hline 06MBW589A & $<0.005$ & - & $<0.2$ & 0.03 & 5 & $<10$ & 10 & $<0.5$ & $<2$ & $>25.0$ & $<0.5$ & $<1$ & 2 & 1 & - & 0.13 & $<10$ & $<1$ & 0.72 \\
\hline 06MBW594A & $<0.005$ & - & $<0.2$ & 0.03 & 9 & $<10$ & 10 & $<0.5$ & $<2$ & $>25.0$ & $<0.5$ & $<1$ & 1 & 1 & - & 0.23 & $<10$ & $<1$ & 0.69 \\
\hline 06MBW615A & $<0.005$ & - & $<0.2$ & 0.55 & 5 & $<10$ & 30 & $<0.5$ & $<2$ & 21.50 & $<0.5$ & 7 & 11 & 14 & - & 2.05 & $<10$ & $<1$ & 0.53 \\
\hline 06MBW619B & $<0.005$ & - & $<0.2$ & 1.09 & 8 & $<10$ & 10 & $<0.5$ & $<2$ & 1.89 & $<0.5$ & 2 & 33 & 2 & - & 2.08 & $<10$ & 1 & 0.54 \\
\hline 06MBW628A & $<0.005$ & - & $<0.2$ & 0.26 & 68 & $<10$ & 20 & $<0.5$ & $<2$ & $>25.0$ & $<0.5$ & 5 & 5 & 19 & - & 1.71 & $<10$ & 2 & 2.42 \\
\hline 06MBW631A & $<0.005$ & - & $<0.2$ & 0.13 & 13 & $<10$ & 10 & $<0.5$ & $<2$ & $>25.0$ & $<0.5$ & 2 & 3 & 9 & - & 1.17 & $<10$ & $<1$ & 0.86 \\
\hline 06MBW646A & $<0.005$ & - & 0.2 & 0.93 & 29 & $<10$ & 20 & 0.5 & $<2$ & 19.50 & $<0.5$ & 7 & 22 & 1 & - & 5.44 & $<10$ & $<1$ & 0.35 \\
\hline 06MBW647A & $<0.005$ & - & $<0.2$ & 0.23 & 2 & $<10$ & 10 & $<0.5$ & $<2$ & 14.00 & $<0.5$ & 2 & 8 & 4 & - & 2.57 & $<10$ & $<1$ & 0.26 \\
\hline 06MBW702B & $<0.005$ & - & $<0.2$ & 0.11 & 15 & $<10$ & 10 & $<0.5$ & $<2$ & 4.87 & $<0.5$ & 2 & 7 & 1 & - & 1.45 & $<10$ & $<1$ & 0.18 \\
\hline 06MBW703A & 0.020 & - & $<0.2$ & 0.07 & 10 & $<10$ & 10 & $<0.5$ & $<2$ & 0.28 & $<0.5$ & 2 & 12 & 2 & - & 1.07 & $<10$ & $<1$ & 0.54 \\
\hline 06MBW711A & $<0.005$ & - & $<0.2$ & 0.13 & 39 & $<10$ & 10 & $<0.5$ & $<2$ & 0.93 & $<0.5$ & 6 & 12 & 2 & - & 1.42 & $<10$ & $<1$ & 0.18 \\
\hline 06MBW711B & $<0.005$ & - & $<0.2$ & 0.49 & $<2$ & $<10$ & $<10$ & $<0.5$ & $<2$ & 0.47 & $<0.5$ & 2 & 18 & 1 & - & 0.88 & $<10$ & $<1$ & 0.18 \\
\hline 06MBW729B & $<0.005$ & - & $<0.2$ & 0.74 & 7 & $<10$ & 20 & $<0.5$ & $<2$ & 0.07 & $<0.5$ & 11 & 23 & 13 & - & 1.89 & $<10$ & $<1$ & 0.20 \\
\hline 06MBW736A & $<0.005$ & - & $<0.2$ & 0.08 & 6 & $<10$ & 20 & $<0.5$ & $<2$ & 0.08 & $<0.5$ & 4 & 43 & 8 & - & 1.26 & $<10$ & 4 & 4.88 \\
\hline 06MBW760A & $<0.005$ & - & 0.2 & 0.88 & 4 & $<10$ & 30 & $<0.5$ & $<2$ & 0.15 & 0.5 & 7 & 32 & 8 & - & 1.06 & $<10$ & 2 & 2.40 \\
\hline 06MBW776A & $<0.005$ & - & $<0.2$ & 0.32 & 2 & $<10$ & 60 & $<0.5$ & $<2$ & 22.00 & $<0.5$ & 2 & 4 & 1 & - & 1.74 & $<10$ & 1 & 2.03 \\
\hline 06MBW777B & 0.010 & - & 0.8 & 0.36 & 570 & $<10$ & 150 & $<0.5$ & 4 & 0.52 & $<0.5$ & 23 & 6 & 59 & - & 38.00 & 10 & 1 & 0.40 \\
\hline 06MBW780A & $<0.005$ & - & $<0.2$ & 0.16 & 7 & $<10$ & 10 & $<0.5$ & $<2$ & 0.05 & $<0.5$ & 2 & 35 & 4 & - & 2.08 & $<10$ & $<1$ & 0.57 \\
\hline 06JEA36B & $<0.005$ & - & $<0.2$ & 0.02 & 6 & $<10$ & $<10$ & $<0.5$ & $<2$ & 11.15 & $<0.5$ & 2 & 22 & 2 & - & 0.18 & $<10$ & $<1$ & 0.52 \\
\hline 06JEA44B & $<0.005$ & - & $<0.2$ & 0.01 & $<2$ & $<10$ & $<10$ & $<0.5$ & $<2$ & 0.20 & $<0.5$ & 1 & 65 & $<1$ & - & 0.13 & $<10$ & $<1$ & 0.24 \\
\hline 06JEA55B & $<0.005$ & - & $<0.2$ & 0.54 & 2 & $<10$ & 20 & $<0.5$ & $<2$ & 0.06 & $<0.5$ & 4 & 49 & 3 & - & 1.08 & $<10$ & $<1$ & 0.21 \\
\hline 06JEA92B & $<0.005$ & - & $<0.2$ & 0.04 & $<2$ & $<10$ & 20 & $<0.5$ & $<2$ & 24.20 & $<0.5$ & $<1$ & 9 & 16 & - & 0.42 & $<10$ & $<1$ & 0.51 \\
\hline 06JEA151A & $<0.005$ & - & 0.2 & 1.82 & 5 & $<10$ & 30 & $<0.5$ & $<2$ & 0.22 & $<0.5$ & 5 & 48 & 10 & - & 5.17 & 10 & $<1$ & 0.18 \\
\hline 06JEA158B & $<0.005$ & - & $<0.2$ & 0.07 & 20 & $<10$ & 30 & $<0.5$ & $<2$ & 21.20 & $<0.5$ & 2 & 9 & 4 & - & 0.63 & $<10$ & $<1$ & 0.45 \\
\hline 06JEA161B & $<0.005$ & - & $<0.2$ & 0.01 & $<2$ & $<10$ & $<10$ & $<0.5$ & $<2$ & 10.05 & $<0.5$ & $<1$ & 31 & $<1$ & - & 0.10 & $<10$ & $<1$ & 0.25 \\
\hline 06JEA164A & $<0.005$ & - & $<0.2$ & 0.01 & 3 & $<10$ & 10 & $<0.5$ & $<2$ & $>25.0$ & $<0.5$ & $<1$ & 2 & $<1$ & - & 0.05 & $<10$ & $<1$ & 0.13 \\
\hline 06JEA165B & 0.022 & - & 0.4 & 0.44 & 1140 & $<10$ & 20 & $<0.5$ & $<2$ & 0.28 & 1.2 & 1 & 46 & 258 & - & 6.72 & $<10$ & 1 & 2.26 \\
\hline 06JEA191A & $<0.005$ & - & $<0.2$ & 0.03 & 19 & $<10$ & $<10$ & $<0.5$ & $<2$ & 0.53 & $<0.5$ & 3 & 48 & 33 & - & 0.38 & $<10$ & $<1$ & 0.10 \\
\hline
\end{tabular}


Table 2. (continued)

\begin{tabular}{|c|c|c|c|c|c|c|c|c|c|c|c|c|c|c|c|c|c|c|c|}
\hline Sample Number & $\begin{array}{l}\mathrm{K} \\
\% \\
\end{array}$ & $\begin{array}{c}\mathrm{La} \\
\mathrm{ppm}\end{array}$ & $\begin{array}{c}\mathrm{Mg} \\
\% \\
\end{array}$ & $\begin{array}{c}\mathrm{Mn} \\
\mathrm{ppm}\end{array}$ & $\begin{array}{c}\text { Mo } \\
\text { ppm }\end{array}$ & $\begin{array}{c}\mathrm{Na} \\
\% \\
\end{array}$ & $\begin{array}{c}\mathrm{Ni} \\
\mathrm{ppm}\end{array}$ & $\begin{array}{l}\mathrm{P} \\
\% \\
\end{array}$ & $\begin{array}{c}\mathrm{Pb} \\
\mathrm{ppm}\end{array}$ & $\begin{array}{l}\mathrm{S} \\
\% \\
\end{array}$ & $\begin{array}{c}\mathrm{Sb} \\
\mathrm{ppm}\end{array}$ & $\begin{array}{c}\mathrm{Sc} \\
\mathrm{ppm}\end{array}$ & $\begin{array}{c}\mathrm{Sr} \\
\mathrm{ppm}\end{array}$ & $\begin{array}{l}\mathrm{Ti} \\
\% \\
\end{array}$ & $\begin{array}{c}\mathrm{TI} \\
\mathrm{ppm}\end{array}$ & $\begin{array}{c}\mathrm{U} \\
\mathrm{ppm}\end{array}$ & $\begin{array}{c}\mathrm{V} \\
\mathrm{ppm}\end{array}$ & $\begin{array}{c}\mathrm{W} \\
\mathrm{ppm}\end{array}$ & $\begin{array}{c}\mathrm{Zn} \\
\mathrm{ppm}\end{array}$ \\
\hline 06MBW472A & 0.04 & $<10$ & 0.74 & 118 & $<1$ & 0.01 & 14 & 70 & 5 & 0.01 & $<2$ & $<1$ & 98 & $<0.01$ & $<10$ & $<10$ & 3 & $<10$ & - \\
\hline 06MBW484A & 12 & $<10$ & 0.13 & 44 & 13 & .02 & 24 & 170 & 24 & 0.06 & 3 & & 19 & 0.01 & $<10$ & $<10$ & 60 & 10 & 53 \\
\hline $06 \mathrm{Ml}$ & 01 & $<10$ & 0.23 & 353 & $<1$ & 01 & & 20 & & 0.02 & 2 & & 176 & .01 & $<10$ & $<10$ & 1 & $=10$ & \\
\hline $06 \mathrm{Ml}$ & 16 & $<10$ & 0.03 & 28 & 32 & 0.05 & 20 & 120 & 27 & 0.98 & $<2$ & & 10 & 0.01 & $<10$ & $<10$ & 25 & $<10$ & 19 \\
\hline $06 \mathrm{M}$ & 16 & 10 & 1.16 & 215 & 2 & .02 & 35 & 180 & 10 & 0.08 & $<2$ & & 13 & 0.01 & $<10$ & $<10$ & 1 & $<10$ & 79 \\
\hline 06MBV & 10 & $<10$ & 0.04 & 31 & 3 & .01 & & 20 & 4 & 0.08 & $<2$ & 1 & 3 & $<0.01$ & $<10$ & $<10$ & 45 & $<10$ & \\
\hline 06MBW & 01 & $<10$ & 0.14 & 54 & $<1$ & 0.02 & $<1$ & 100 & 3 & 0.01 & 3 & $<1$ & 280 & $<0.01$ & $<10$ & $<10$ & $<1$ & $<10$ & \\
\hline 06MBW594A & 01 & $<10$ & 0.13 & 171 & $<1$ & 0.0 & $<1$ & 80 & 4 & 0.01 & 3 & 1 & 1230 & $<0.01$ & $<10$ & $<10$ & 1 & $<10$ & \\
\hline 06MBW615A & 17 & 10 & 0.97 & 536 & $<1$ & 0 & 26 & 370 & 10 & $<0.01$ & 3 & 5 & 1470 & $<0.01$ & $<10$ & $<10$ & 8 & $<10$ & 23 \\
\hline 06MBW619B & 03 & $<10$ & 0.58 & 322 & $<1$ & 0.0 & $\varepsilon$ & 40 & & 0.01 & 4 & & 51 & $<0.01$ & $<10$ & $<10$ & 19 & $<10$ & 28 \\
\hline 06MBW628A & 0.05 & $<10$ & 0.14 & 521 & 1 & 0.0 & 39 & 170 & 8 & $<0.01$ & 98 & & 98 & $<0.01$ & $<10$ & $<10$ & 11 & $<10$ & 80 \\
\hline 06MBW631A & 0.04 & $<10$ & 0.14 & 430 & 1 & 0.0 & 11 & 80 & 3 & $<0.01$ & 57 & & 108 & $<0.01$ & $<10$ & $<10$ & 10 & $<10$ & 24 \\
\hline 06MBW646A & 0.05 & 10 & 2.21 & 2990 & $<1$ & 0.03 & 23 & 540 & 4 & $<0.01$ & 0 & s & 501 & $<0.01$ & $<10$ & $<10$ & 18 & $<10$ & 66 \\
\hline 06MBW647A & 0.04 & $<10$ & 2.57 & 1910 & $<1$ & 0.02 & 4 & 230 & 11 & 0.22 & $<2$ & 2 & 564 & $<0.01$ & $<10$ & $<10$ & 5 & $<10$ & 19 \\
\hline 06MBW702B & 0.04 & $<10$ & 0.72 & 244 & 2 & 0.0 & 2 & 50 & & 0.12 & $<2$ & 7 & 140 & $<0.01$ & $<10$ & $<10$ & 1 & $<10$ & 5 \\
\hline 06MBW7 & 0.03 & $<10$ & 0.06 & 152 & $<1$ & 0 & 4 & 80 & 2 & 0.01 & $<2$ & $<1$ & 5 & $<0.01$ & $<10$ & $<10$ & $\perp$ & $<10$ & 7 \\
\hline $06 \mathrm{Ml}$ & 06 & $<10$ & 0.03 & 53 & $?$ & & 7 & 110 & $<2$ & 0.01 & 2 & - & 22 & $<0.01$ & $<10$ & $<10$ & $z$ & $<10$ & $<2$ \\
\hline 06MBW & .01 & $<10$ & 0.55 & 57 & $<1$ & .01 & 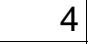 & 10 & $<2$ & $<0.01$ & $<2$ & $<1$ & 10 & $<0.01$ & $<10$ & $<10$ & 7 & 10 & 10 \\
\hline 06MBW729B & 01 & 10 & 0.24 & 121 & 1 & 0 & 35 & 170 & 7 & 0.01 & $<2$ & & 11 & $<0.01$ & $<10$ & $<10$ & 15 & $<10$ & 30 \\
\hline 06MBW736A & 03 & 10 & 0.02 & 660 & $<1$ & & 27 & 70 & 8 & 0.01 & $<2$ & & 3 & $<0.01$ & $<10$ & $<10$ & 2 & $<10$ & \\
\hline 06MBW7 & 0.03 & 10 & 0.46 & 71 & 1 & 0 & 60 & 150 & 5 & 0.01 & $<2$ & 3 & 14 & 0.07 & $<10$ & 10 & 17 & $<10$ & 66 \\
\hline 06MBW776A & 0.01 & 10 & 8.60 & 590 & $<1$ & & & 70 & 4 & $<0.01$ & $<2$ & 1 & 178 & $<0.01$ & $<10$ & $<10$ & 5 & $<10$ & 14 \\
\hline 06MBW777B & 0.04 & $<10$ & 0.25 & 127 & 8 & & 14 & 850 & 34 & 0.17 & $<2$ & & 35 & $<0.01$ & $<10$ & 10 & 29 & $<10$ & 4 \\
\hline $06 \mathrm{M}$ & 01 & $<10$ & 0.06 & 32 & 6 & & 29 & 90 & 8 & 0.01 & $<2$ & & 3 & $<0.01$ & $<10$ & $<10$ & 19 & $<10$ & 24 \\
\hline 0 & 01 & $<10$ & 0.05 & 69 & $<$ & & $<1$ & 50 & 2 & 0.01 & $<2$ & $<$ & 77 & $<0.01$ & $<10$ & $<10$ & $<1$ & 10 & \\
\hline B & $<0.01$ & $<10$ & $<0.01$ & 8 & $<1$ & & 2 & 10 & $<2$ & $<0.01$ & $<2$ & $<1$ & 2 & $<0.01$ & $<10$ & $<10$ & $<1$ & $<10$ & $<2$ \\
\hline EA & 0.07 & 10 & 0. & 89 & $<$ & & 13 & 220 & 0 & $<0.01$ & $<2$ & & 4 & 0.01 & $<10$ & $<10$ & 10 & $<10$ & 17 \\
\hline$B$ & 02 & $<10$ & $0 \perp$ & 37 & $<1$ & & $<1$ & 220 & $y$ & $<0.01$ & $<2$ & 3 & 351 & $<0.01$ & $<10$ & $<10$ & 5 & $<10$ & 11 \\
\hline 06JEA & 10 & 10 & & & 6 & & 22 & 410 & 5 & 0.63 & 2 & & 6 & 0.01 & $<10$ & $<10$ & 48 & $<10$ & 72 \\
\hline 06JEA1 & 2 & $<10$ & 0.13 & 546 & 3 & & $\epsilon$ & 80 & & $<0.01$ & 11 & & 52 & 0.01 & $<10$ & $<10$ & 3 & $<10$ & 15 \\
\hline JEA & 01 & $<10$ & 0.03 & 33 & $<$ & & $<$ & 10 & & 0.01 & $<2$ & $<\perp$ & 97 & 0.01 & $<10$ & $<10$ & $<1$ & $<10$ & 4 \\
\hline JEA1 & $<0.01$ & $<10$ & 0.02 & 61 & $<1$ & & $<1$ & $<1$ & & $<0.01$ & $<2$ & $<1$ & 264 & $<0.01$ & $<10$ & $<10$ & $<1$ & $<10$ & \\
\hline JJEA165B & 0.06 & 10 & 0.02 & 26 & 1 & 0.01 & 12 & 800 & 79 & 0.06 & 165 & 4 & 17 & $<0.01$ & $<10$ & 20 & 48 & $<10$ & 295 \\
\hline 6JEA191A & 0.01 & 10 & .01 & 37 & $<1$ & .01 & 0 & 40 & 4 & 0.01 & $<2$ & $<1$ & & $<0.01$ & 10 & $<10$ & 1 & 10 & \\
\hline
\end{tabular}


Table 2. (continued).

\begin{tabular}{|c|c|c|c|c|c|c|c|c|c|c|c|c|c|c|c|c|c|c|c|}
\hline Sample Number & $\begin{array}{c}\mathrm{Au} \\
\mathrm{ppm}\end{array}$ & $\begin{array}{c}\mathrm{Au}(+) \\
\mathrm{ppm}\end{array}$ & $\begin{array}{c}\mathrm{Ag} \\
\mathrm{ppm}\end{array}$ & $\begin{array}{l}\mathrm{Al} \\
\% \\
\end{array}$ & $\begin{array}{c}\text { As } \\
\text { ppm }\end{array}$ & $\begin{array}{c}\text { B } \\
\text { ppm }\end{array}$ & $\begin{array}{c}\mathrm{Ba} \\
\mathrm{ppm}\end{array}$ & $\begin{array}{c}\mathrm{Be} \\
\mathrm{ppm}\end{array}$ & $\begin{array}{c}\mathrm{Bi} \\
\text { ppm }\end{array}$ & $\begin{array}{l}\mathrm{Ca} \\
\% \\
\end{array}$ & $\begin{array}{c}\text { Cd } \\
\text { ppm }\end{array}$ & $\begin{array}{c}\text { Co } \\
\text { ppm }\end{array}$ & $\begin{array}{c}\mathrm{Cr} \\
\mathrm{ppm}\end{array}$ & $\begin{array}{c}\mathrm{Cu} \\
\mathrm{ppm}\end{array}$ & $\begin{array}{c}\mathrm{Cu}(+) \\
\% \\
\end{array}$ & $\begin{array}{l}\mathrm{Fe} \\
\% \\
\end{array}$ & $\begin{array}{c}\mathrm{Ga} \\
\mathrm{ppm}\end{array}$ & $\begin{array}{c}\mathrm{Hg} \\
\mathrm{ppm}\end{array}$ & $\begin{array}{c}\mathrm{Hg}(+) \\
\mathrm{ppm} \\
\end{array}$ \\
\hline 06JEA196B & 0.005 & - & $<0.2$ & 0.05 & 36 & $<10$ & 10 & $<0.5$ & $<2$ & $>25.0$ & $<0.5$ & 1 & 5 & $<1$ & - & 1.18 & $<10$ & 2 & 0.40 \\
\hline 06JEA334A & 0.006 & - & $<0.2$ & 0.05 & 20 & $<10$ & $<10$ & $<0.5$ & $<2$ & 14.75 & $<0.5$ & 2 & 23 & 20 & - & 0.55 & $<10$ & 2 & 0.29 \\
\hline 06JEA390A & 0.008 & - & $<0.2$ & 0.96 & 5 & $<10$ & 20 & $<0.5$ & $<2$ & 0.47 & $<0.5$ & 12 & 46 & 18 & - & 3.23 & $<10$ & 1 & 0.05 \\
\hline 06JEA419A & 0.007 & - & 0.2 & 0.43 & 136 & $<10$ & 90 & $<0.5$ & 3 & 0.05 & 0.9 & 3 & 35 & 310 & - & 9.16 & $<10$ & 1 & 0.10 \\
\hline 06JEA437A & $<0.005$ & - & $<0.2$ & 0.03 & 8 & $<10$ & 10 & $<0.5$ & $<2$ & $>25.0$ & $<0.5$ & 3 & 4 & 8 & - & 0.39 & $<10$ & 3 & 1.51 \\
\hline 06JEA450B & $<0.005$ & - & $<0.2$ & 0.04 & $<2$ & $<10$ & 60 & $<0.5$ & $<2$ & 0.16 & $<0.5$ & 2 & 75 & 4 & - & 0.22 & $<10$ & $<1$ & 0.24 \\
\hline 06JEA464A & $<0.005$ & - & $<0.2$ & 0.28 & 39 & $<10$ & 40 & $<0.5$ & $<2$ & 0.47 & $<0.5$ & 15 & 47 & 70 & - & 4.53 & $<10$ & 1 & 0.79 \\
\hline 06JEA476A & $<0.005$ & - & $<0.2$ & 0.16 & 16 & $<10$ & $<10$ & $<0.5$ & $<2$ & 8.18 & 0.7 & 2 & 35 & 3 & - & 1.73 & $<10$ & $<1$ & 0.04 \\
\hline 06JEA481A & $<0.005$ & - & $<0.2$ & 0.17 & 23 & $<10$ & 10 & $<0.5$ & $<2$ & $>25.0$ & $<0.5$ & 4 & 9 & 3 & - & 0.95 & $<10$ & 3 & 0.24 \\
\hline 06JEA487A & $<0.005$ & - & $<0.2$ & 0.25 & $<2$ & $<10$ & 10 & $<0.5$ & $<2$ & 6.24 & $<0.5$ & 1 & 34 & $<1$ & - & 0.60 & $<10$ & $<1$ & 0.14 \\
\hline 06JEA512A & $<0.005$ & - & $<0.2$ & 0.03 & $<2$ & $<10$ & $<10$ & $<0.5$ & $<2$ & 2.51 & $<0.5$ & 2 & 48 & 9 & - & 0.15 & $<10$ & $<1$ & 0.13 \\
\hline 06JEA513A & $<0.005$ & - & $<0.2$ & 0.02 & $<2$ & $<10$ & $<10$ & $<0.5$ & $<2$ & 1.36 & $<0.5$ & 1 & 55 & $<1$ & - & 0.26 & $<10$ & $<1$ & 0.03 \\
\hline 06JEA513B & 0.038 & - & 0.3 & 0.42 & 1100 & $<10$ & 10 & $<0.5$ & 10 & 1.00 & 0.5 & 93 & 14 & 35 & - & 25.70 & $<10$ & 1 & 0.11 \\
\hline 06JEA518A & $<0.005$ & - & $<0.2$ & 0.04 & 11 & $<10$ & $<10$ & $<0.5$ & $<2$ & 0.60 & $<0.5$ & 2 & 53 & 9 & - & 0.50 & $<10$ & 1 & 0.04 \\
\hline 06JEA522A & 0.028 & - & 0.4 & 0.10 & 9 & $<10$ & $<10$ & $<0.5$ & $<2$ & 0.68 & $<0.5$ & 7 & 41 & $>10000$ & 6.98 & 1.14 & $<10$ & $<1$ & 0.30 \\
\hline 06JEA522B & 0.027 & - & 0.2 & 0.09 & 2 & $<10$ & $<10$ & $<0.5$ & $<2$ & 0.08 & $<0.5$ & 5 & 46 & $>10000$ & 2.54 & 1.59 & $<10$ & $<1$ & $<0.1$ \\
\hline 06JEA523A & $<0.005$ & - & $<0.2$ & 0.02 & $<2$ & $<10$ & $<10$ & $<0.5$ & $<2$ & 0.11 & $<0.5$ & 1 & 62 & 271 & - & 0.13 & $<10$ & $<1$ & 0.05 \\
\hline 06JEA524A & $<0.005$ & - & $<0.2$ & 0.03 & 2 & $<10$ & 10 & $<0.5$ & $<2$ & $>25.0$ & $<0.5$ & 1 & 3 & 36 & - & 0.25 & $<10$ & 2 & 0.06 \\
\hline 06JEA575A & $<0.005$ & - & $<0.2$ & 0.64 & $<2$ & $<10$ & 30 & $<0.5$ & $<2$ & 0.51 & $<0.5$ & 6 & 46 & 21 & - & 1.16 & $<10$ & $<1$ & 0.08 \\
\hline 06JEA663B & 0.005 & - & $<0.2$ & 0.59 & 160 & $<10$ & 20 & $<0.5$ & 5 & 3.44 & 1.0 & 21 & 63 & 199 & - & 12.55 & 10 & 34 & 34.40 \\
\hline 06JEA691A & $<0.005$ & - & $<0.2$ & 0.05 & 9 & $<10$ & 10 & $<0.5$ & $<2$ & $>25.0$ & $<0.5$ & 1 & 3 & 6 & - & 0.26 & $<10$ & 2 & 0.46 \\
\hline 06JEA691D & $<0.005$ & - & 0.3 & 0.16 & 267 & 110 & 100 & 1.5 & 2 & 11.95 & $<0.5$ & 3 & 30 & 17 & - & 9.36 & $<10$ & 15 & 16.50 \\
\hline 06JEA705A & $<0.005$ & - & $<0.2$ & 1.20 & 670 & $<10$ & 40 & $<0.5$ & $<2$ & 0.94 & 0.6 & 15 & 38 & 31 & - & 3.12 & $<10$ & $<1$ & 0.08 \\
\hline 06JEA706A & 6.150 & - & 1.2 & 1.70 & $>10000$ & $<10$ & 20 & 0.5 & 2 & 0.62 & $<0.5$ & 24 & 62 & 4 & - & 9.77 & 10 & 2 & 0.10 \\
\hline 06RN90B & $<0.005$ & - & 0.2 & 0.25 & 40 & $<10$ & 190 & $<0.5$ & $<2$ & 0.03 & $<0.5$ & 7 & 22 & 31 & - & 1.38 & $<10$ & 11 & 12.50 \\
\hline 06RN202C & $<0.005$ & - & $<0.2$ & 0.09 & 9 & $<10$ & 10 & $<0.5$ & $<2$ & 0.65 & $<0.5$ & 3 & 46 & 4 & - & 0.81 & $<10$ & 4 & 5.13 \\
\hline 06RN207C & $<0.005$ & - & $<0.2$ & 0.05 & 11 & $<10$ & 10 & $<0.5$ & $<2$ & 3.01 & $<0.5$ & 2 & 50 & $<1$ & - & 0.41 & $<10$ & 1 & 0.83 \\
\hline 06RN244A & 0.024 & - & 1.2 & 0.27 & 80 & $<10$ & 20 & $<0.5$ & $<2$ & 0.04 & $<0.5$ & 5 & 9 & 21 & - & 0.92 & $<10$ & 4 & 5.19 \\
\hline 06RN245A & 0.167 & - & 1.9 & 0.53 & 1675 & $<10$ & 40 & $<0.5$ & $<2$ & 0.08 & 8.2 & 8 & 13 & 166 & - & 1.78 & $<10$ & 11 & 11.2 \\
\hline 06RN245C & 0.020 & - & 1.4 & 0.40 & 193 & $<10$ & 30 & $<0.5$ & $<2$ & 0.06 & 1.2 & 3 & 12 & 41 & - & 1.21 & $<10$ & 2 & 2.70 \\
\hline 06RN246A & 0.025 & - & 0.2 & 2.63 & 26 & $<10$ & 90 & 1.0 & 2 & 2.43 & $<0.5$ & 24 & 28 & 25 & - & 4.69 & $<10$ & $<1$ & 0.05 \\
\hline 06RN248B & 0.012 & - & $<0.2$ & 2.31 & 10 & $<10$ & 260 & 1.3 & $<2$ & 2.80 & $<0.5$ & 27 & 64 & 26 & - & 4.93 & 10 & $<1$ & 0.04 \\
\hline 06RN249A & 0.010 & - & $<0.2$ & 4.22 & 5 & $<10$ & 80 & 0.8 & $<2$ & 3.19 & $<0.5$ & 40 & 69 & 22 & - & 5.32 & 10 & 1 & 0.48 \\
\hline 06RN283A & $<0.005$ & - & 0.2 & 0.96 & $<2$ & $<10$ & 10 & $<0.5$ & $<2$ & 0.21 & $<0.5$ & 8 & 74 & 9 & - & 1.66 & $<10$ & 1 & 0.32 \\
\hline
\end{tabular}


Table 2. (continued)

\begin{tabular}{|c|c|c|c|c|c|c|c|c|c|c|c|c|c|c|c|c|c|c|c|}
\hline Sample Number & $\begin{array}{l}\mathrm{K} \\
\% \\
\end{array}$ & $\begin{array}{c}\mathrm{La} \\
\mathrm{ppm}\end{array}$ & $\begin{array}{c}\mathrm{Mg} \\
\% \\
\end{array}$ & $\begin{array}{c}\mathrm{Mn} \\
\mathrm{ppm}\end{array}$ & $\begin{array}{c}\text { Mo } \\
\text { ppm }\end{array}$ & $\begin{array}{c}\mathrm{Na} \\
\% \\
\end{array}$ & $\begin{array}{c}\mathrm{Ni} \\
\mathrm{ppm}\end{array}$ & $\begin{array}{l}\mathrm{P} \\
\% \\
\end{array}$ & $\begin{array}{c}\mathrm{Pb} \\
\mathrm{ppm}\end{array}$ & $\begin{array}{l}\mathrm{S} \\
\% \\
\end{array}$ & $\begin{array}{c}\text { Sb } \\
\text { ppm }\end{array}$ & $\begin{array}{c}\text { Sc } \\
\text { ppm }\end{array}$ & $\begin{array}{c}\mathrm{Sr} \\
\mathrm{ppm}\end{array}$ & $\begin{array}{l}\mathrm{Ti} \\
\% \\
\end{array}$ & $\begin{array}{c}\text { TI } \\
\text { ppm }\end{array}$ & $\begin{array}{c}\text { U } \\
\text { ppm }\end{array}$ & $\begin{array}{c}\mathrm{V} \\
\mathrm{ppm}\end{array}$ & $\begin{array}{c}\text { W } \\
\text { ppm }\end{array}$ & $\begin{array}{c}\mathrm{Zn} \\
\mathrm{ppm}\end{array}$ \\
\hline 06JEA196B & 0.01 & $=10$ & 0.11 & 204 & $<1$ & 0.01 & 2 & 40 & 8 & $<0.01$ & 19 & $<1$ & 422 & $<0.01$ & $<10$ & $<10$ & 3 & $<10$ & 11 \\
\hline 06JEA334A & 0.01 & 10 & 10 & 111 & 1 & 0.01 & 15 & 110 & 4 & $<0.01$ & 13 & $<1$ & 93 & 0.01 & $<10$ & $<10$ & ( & $<10$ & \\
\hline 06JEA390A & 0.07 & 10 & 56 & 260 & 1 & 0.03 & 35 & 570 & 13 & 0.08 & 2 & & 9 & 0.01 & $<10$ & $<10$ & 32 & $<10$ & 24 \\
\hline 06JEA419A & 0.08 & $<10$ & 0.01 & 20 & 40 & 0.01 & 54 & 590 & 14 & 0.04 & 14 & 2 & 5 & $<0.01$ & $<10$ & $<10$ & 63 & $<10$ & 194 \\
\hline EA437A & 0.01 & $=10$ & .13 & 439 & $<1$ & 0.01 & 19 & 150 & 4 & $<0.01$ & 3 & 1 & 109 & $<0.01$ & $<10$ & $<10$ & & $<10$ & 19 \\
\hline EA450B & $<0.01$ & $<10$ & 0.01 & 11 & $<1$ & 0.01 & 6 & 20 & 2 & $<0.01$ & $<2$ & $<1$ & 2 & $<0.01$ & 10 & $<10$ & & $<10$ & \\
\hline 06JEA464A & 0.06 & 20 & 0.51 & 344 & $<1$ & 0.02 & 50 & 530 & 8 & 0.75 & 3 & & 18 & $<0.01$ & $<10$ & $<10$ & 42 & $<10$ & 69 \\
\hline 06JEA476A & 0.01 & $<10$ & 1.52 & 832 & $<1$ & 0.02 & 9 & 70 & 6 & 0.01 & 8 & 2 & 181 & $<0.01$ & $<10$ & $<10$ & & $<10$ & 70 \\
\hline 06JEA481A & 0.04 & $<10$ & 0.46 & 826 & $<1$ & 0.01 & 12 & 190 & 10 & $<0.01$ & $<2$ & 3 & 2030 & 0.01 & $<10$ & $<10$ & & $<10$ & \\
\hline 06JEA487A & 0.02 & 10 & 0.22 & 477 & $<1$ & 0.03 & 7 & 90 & 9 & 0.01 & 5 & 2 & 156 & $<0.01$ & $<10$ & $<10$ & 2 & $<10$ & 7 \\
\hline 06JEA512A & 0.01 & $<10$ & 0.01 & 91 & $<1$ & 0.01 & 3 & 10 & 3 & 0.01 & $<2$ & $<1$ & 36 & $<0.01$ & $<10$ & $<10$ & $<1$ & $<10$ & $<2$ \\
\hline 06JEA513A & 0.01 & $<10$ & 0.01 & 103 & $<1$ & $<0.01$ & 3 & 10 & 3 & $<0.01$ & $<2$ & $<1$ & 10 & $<0.01$ & $<10$ & $<10$ & $<1$ & $<10$ & $<2$ \\
\hline 06JEA513B & 0.09 & 10 & 0.10 & 43 & 2 & 0.01 & 222 & 3520 & 64 & 0.02 & 10 & 1 & 23 & 0.05 & $<10$ & $<10$ & & $<10$ & 2 \\
\hline 06JEA518A & 0.01 & $<10$ & $<0.01$ & 59 & $<1$ & $<0.01$ & 7 & 70 & 2 & $<0.01$ & 2 & $<1$ & 16 & $<0.01$ & $<10$ & $<10$ & & $<10$ & $<2$ \\
\hline 06JEA522A & 0.01 & $<10$ & 01 & 240 & 1 & $<0.01$ & 7 & 160 & $<2$ & 0.01 & 7 & 1 & 16 & $<0.01$ & $<10$ & $<10$ & & 10 & 22 \\
\hline 06JEA522B & 0.03 & 10 & .01 & 121 & $<1$ & $<0.01$ & 11 & 420 & 5 & 0.01 & 6 & $<1$ & 7 & $<0.01$ & $<10$ & $<10$ & & $<10$ & 59 \\
\hline 06JEA & 0.01 & $<10$ & 0.01 & 13 & $<1$ & 0.01 & 3 & $<10$ & $<2$ & $<0.01$ & 2 & $<1$ & 2 & $<0.01$ & $<10$ & $<10$ & $<1$ & $<10$ & $<2$ \\
\hline 06JEA524A & .01 & $<10$ & 0.10 & 193 & $<1$ & 0.01 & 3 & 100 & 7 & $<0.01$ & 5 & $<1$ & 669 & $<0.01$ & 10 & $<10$ & & $<10$ & $<2$ \\
\hline JEA575A & 16 & 10 & 20 & 98 & $<1$ & 0.01 & 14 & 230 & $<2$ & 0.07 & $<2$ & 1 & 11 & 0.01 & $<10$ & $<10$ & 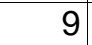 & $<10$ & 9 \\
\hline JEA663B & 0.06 & $<10$ & .08 & 188 & 0 & 0.01 & 77 & 1140 & 18 & 0.02 & 187 & 2 & 17 & $<0.01$ & 10 & 10 & 10 & $<10$ & 129 \\
\hline JEA691A & 0.01 & $<10$ & 4.42 & 100 & $<1$ & 0.01 & 4 & 40 & 5 & $<0.01$ & 12 & $<1$ & 103 & $<0.01$ & $<10$ & $<10$ & $\mathrm{v}$ & $<10$ & 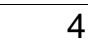 \\
\hline JEAC & 02 & $<10$ & 0.78 & 826 & 1 & 0.01 & 8 & 1930 & 18 & 0.01 & 210 & $<1$ & 38 & $<0.01$ & $<10$ & $<10$ & 30 & $<10$ & 128 \\
\hline JEA7 & 22 & 20 & 68 & 417 & 1 & 0.16 & 61 & 680 & 14 & 0.02 & 9 & & 27 & 0.01 & $<10$ & $<10$ & 7 & $<10$ & 58 \\
\hline 06JEA7 & & 10 & 55 & 366 & 3 & 0.03 & 48 & 420 & 13 & 3.43 & 16 & 5 & 27 & 0.01 & $<10$ & $<10$ & 56 & $<10$ & 40 \\
\hline PRNS & & 10 & 0 & 28 & 11 & 0.01 & 12 & 260 & 13 & $<0.01$ & 17 & & 8 & $<0.01$ & $<10$ & $<10$ & 11 & $<10$ & 16 \\
\hline 06RN2 & 4 & $<10$ & 2 & 159 & $<1$ & $<0.01$ & 5 & 60 & 3 & 0.01 & $\angle$ & $<1$ & 13 & $<0.01$ & $<10$ & $<10$ & & $<10$ & \\
\hline 06RN2 & 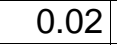 & $<$ & 4 & 169 & $<1$ & 0. & 3 & 10 & 0 & 0.01 & $<2$ & $<1$ & 93 & $<0.01$ & $<10$ & $<10$ & & $<10$ & \\
\hline 06RN244A & 08 & $<10$ & 03 & 80 & 1 & 0. & 8 & 50 & 110 & 0.01 & \multicolumn{2}{|c|}{$7<<1$} & 4 & $<0.01$ & $<10$ & $<10$ & 1 & $<10$ & 238 \\
\hline 06RN2 & & 10 & 34 & 99 & 2 & 0 & 22 & 170 & 797 & 0.04 & 106 & 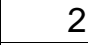 & 16 & $<0.01$ & $<10$ & $<10$ & 12 & $<10$ & 1080 \\
\hline 06RN2 & 12 & $<10$ & 0 & 80 & 1 & 0 & 9 & 60 & 165 & 0.02 & 23 & $<1$ & 7 & 0.01 & $<10$ & $<10$ & 7 & $<10$ & 505 \\
\hline 06RN246A & 23 & 40 & 2 & 620 & 1 & 0. & 36 & 970 & 9 & 0.12 & 4 & 7 & 223 & 0.14 & $<10$ & $<10$ & 32 & $<10$ & 100 \\
\hline 06RN248B & 42 & 50 & 2.26 & 6 & 1 & 0.2 & 69 & 3190 & 8 & 0.18 & 6 & & 191 & 0.3 & $<10$ & $<10$ & $4 \pi$ & $<10$ & 176 \\
\hline 06RN249A & 0.16 & 20 & 3.02 & 746 & 1 & 0.51 & 73 & 1680 & 7 & 0.16 & 44 & 11 & 235 & 0.16 & $<10$ & $<10$ & 74 & $<10$ & 300 \\
\hline $3 A$ & 0.02 & 10 & 79 & 363 & $=1$ & .12 & 32 & 400 & & 0.01 & & 2 & & 0.03 & $=10$ & 10 & 20 & 10 & \\
\hline
\end{tabular}


Table 2. (continued).

\begin{tabular}{|c|c|c|c|c|c|c|c|c|c|c|c|c|c|c|c|c|c|c|c|}
\hline Sample Number & $\begin{array}{c}\text { Au } \\
\text { ppm }\end{array}$ & $\begin{array}{c}\mathrm{Au}(+) \\
\mathrm{ppm}\end{array}$ & $\begin{array}{c}\mathrm{Ag} \\
\mathrm{ppm}\end{array}$ & $\begin{array}{l}\mathrm{Al} \\
\% \\
\end{array}$ & $\begin{array}{c}\text { As } \\
\text { ppm }\end{array}$ & $\begin{array}{c}\mathrm{B} \\
\mathrm{ppm}\end{array}$ & $\begin{array}{c}\mathrm{Ba} \\
\mathrm{ppm}\end{array}$ & $\begin{array}{c}\mathrm{Be} \\
\mathrm{ppm}\end{array}$ & $\begin{array}{c}\mathrm{Bi} \\
\mathrm{ppm}\end{array}$ & $\begin{array}{c}\mathrm{Ca} \\
\% \\
\end{array}$ & $\begin{array}{c}\mathrm{Cd} \\
\mathrm{ppm}\end{array}$ & $\begin{array}{c}\text { Co } \\
\mathrm{ppm}\end{array}$ & $\begin{array}{c}\mathrm{Cr} \\
\mathrm{ppm}\end{array}$ & $\begin{array}{c}\mathrm{Cu} \\
\mathrm{ppm}\end{array}$ & $\begin{array}{c}\mathrm{Cu}(+) \\
\% \\
\end{array}$ & $\begin{array}{c}\mathrm{Fe} \\
\% \\
\end{array}$ & $\begin{array}{c}\mathrm{Ga} \\
\mathrm{ppm}\end{array}$ & $\begin{array}{c}\mathrm{Hg} \\
\mathrm{ppm}\end{array}$ & $\begin{array}{c}\mathrm{Hg}(+) \\
\mathrm{ppm}\end{array}$ \\
\hline 06RN284A & $<0.005$ & - & $<0.2$ & 0.43 & 4 & $<10$ & 30 & $<0.5$ & $<2$ & 2.28 & $<0.5$ & 3 & 42 & 75 & - & 0.68 & $<10$ & 1 & 0.33 \\
\hline 06RN327B & $<0.005$ & - & $<0.2$ & 0.03 & 12 & $<10$ & 10 & $<0.5$ & $<2$ & 0.13 & $<0.5$ & 1 & 64 & 1 & - & 0.33 & $<10$ & $<1$ & 1.65 \\
\hline 06RN328A & 0.005 & - & $<0.2$ & 0.05 & 18 & $<10$ & 10 & $<0.5$ & $<2$ & 0.01 & $<0.5$ & 1 & 61 & $<1$ & - & 0.45 & $<10$ & 1 & 1.77 \\
\hline 06RN337C & 0.009 & - & 0.2 & 0.03 & 35 & $<10$ & 30 & $<0.5$ & 2 & 0.13 & $<0.5$ & 1 & 54 & 6 & - & 6.99 & $<10$ & 46 & 45.10 \\
\hline 06RN343C & $<0.005$ & - & $<0.2$ & 0.25 & 5 & $<10$ & 20 & $<0.5$ & $<2$ & 0.52 & $<0.5$ & 2 & 59 & 5 & - & 0.48 & $<10$ & $<1$ & 0.61 \\
\hline 06RN522A & 0.008 & - & $<0.2$ & 3.47 & 52 & $<10$ & 730 & 1.3 & 2 & 4.23 & $<0.5$ & 25 & 128 & 29 & - & 5.75 & 10 & 1 & 0.55 \\
\hline 06RN556B & $<0.005$ & - & $<0.2$ & 0.24 & 7 & $<10$ & 20 & $<0.5$ & $<2$ & 0.52 & $<0.5$ & 3 & 51 & 5 & - & 0.48 & $<10$ & 1 & 0.58 \\
\hline 06RN574B & $<0.005$ & - & 0.3 & 0.31 & 31 & $<10$ & 190 & $<0.5$ & $<2$ & 0.01 & $<0.5$ & 3 & 44 & 25 & - & 1.63 & $<10$ & $<1$ & 0.23 \\
\hline
\end{tabular}


Table 2. (continued)

\begin{tabular}{|c|c|c|c|r|r|r|r|r|r|r|r|r|r|r|r|r|r|r|r|}
\hline Sample Number & $\begin{array}{c}\mathrm{K} \\
\%\end{array}$ & $\begin{array}{c}\mathrm{La} \\
\mathrm{ppm}\end{array}$ & $\begin{array}{c}\mathrm{Mg} \\
\%\end{array}$ & $\begin{array}{c}\mathrm{Mn} \\
\mathrm{ppm}\end{array}$ & $\begin{array}{c}\mathrm{Mo} \\
\mathrm{ppm}\end{array}$ & $\begin{array}{c}\mathrm{Na} \\
\%\end{array}$ & $\begin{array}{c}\mathrm{Ni} \\
\mathrm{ppm}\end{array}$ & $\begin{array}{c}\mathrm{P} \\
\%\end{array}$ & $\begin{array}{c}\mathrm{Pb} \\
\mathrm{ppm}\end{array}$ & $\begin{array}{c}\mathrm{S} \\
\%\end{array}$ & $\begin{array}{c}\mathrm{Sb} \\
\mathrm{ppm}\end{array}$ & $\begin{array}{c}\mathrm{Sc} \\
\mathrm{ppm}\end{array}$ & $\begin{array}{c}\mathrm{Sr} \\
\mathrm{ppm}\end{array}$ & $\begin{array}{c}\mathrm{Ti} \\
\%\end{array}$ & $\begin{array}{c}\mathrm{TI} \\
\mathrm{ppm}\end{array}$ & $\begin{array}{c}\mathrm{U} \\
\mathrm{ppm}\end{array}$ & $\begin{array}{c}\mathrm{V} \\
\mathrm{ppm}\end{array}$ & $\begin{array}{c}\mathrm{W} \\
\mathrm{ppm}\end{array}$ & $\begin{array}{c}\mathrm{Zn} \\
\mathrm{ppm}\end{array}$ \\
\hline 06RN284A & 0.04 & $<10$ & 0.30 & 847 & $<1$ & 0.01 & 7 & 690 & 6 & $<0.01$ & $<2$ & 1 & 140 & 0.02 & $<10$ & $<10$ & 9 & $<10$ & 11 \\
\hline 06RN327B & 0.01 & $<10$ & $<0.01$ & 19 & 1 & $<0.01$ & 4 & 20 & 5 & 0.01 & 8 & $<1$ & 3 & $<0.01$ & $<10$ & $<10$ & 2 & $<10$ & 2 \\
\hline 06RN328A & 0.05 & $<10$ & $<0.01$ & 9 & 1 & $<0.01$ & 3 & 60 & 15 & 0.08 & 8 & $<1$ & 13 & $<0.01$ & $<10$ & $<10$ & 2 & $<10$ & 5 \\
\hline 06RN337C & 0.56 & $<10$ & 0.02 & 13 & 4 & 0.02 & 3 & 280 & 18 & 1.16 & 193 & $<1$ & 9 & $<0.01$ & 20 & $<10$ & 8 & $<10$ & 4 \\
\hline 06RN343C & 0.07 & $<10$ & 0.06 & 235 & 1 & 0.03 & 7 & 60 & 28 & 0.02 & 8 & $<1$ & 13 & $<0.01$ & $<10$ & $<10$ & 3 & $<10$ & 36 \\
\hline 06RN522A & 0.31 & 70 & 2.09 & 823 & 2 & 0.29 & 59 & 2910 & 18 & 0.22 & 15 & 16 & 164 & 0.32 & $<10$ & $<10$ & 95 & $<10$ & 133 \\
\hline 06RN556B & 0.07 & $<10$ & 0.06 & 236 & 1 & 0.03 & 8 & 60 & 31 & 0.01 & 11 & $<1$ & 12 & $<0.01$ & $<10$ & $<10$ & 3 & $<10$ & 36 \\
\hline 06RN574B & 0.13 & 10 & 0.02 & 12 & 19 & 0.01 & 22 & 280 & 41 & 0.02 & 11 & 1 & 24 & $<0.01$ & $<10$ & $<10$ & 38 & $<10$ & 53 \\
\hline
\end{tabular}


Table 3. Location and description of rocks collected for major-oxide, minor-oxide, and trace-element analyses in the Solomon and Nome Quadrangles, Seward Peninsula, Alaska.

\begin{tabular}{|c|c|c|c|c|c|}
\hline Sample Number & Latitude & Longitude & UTM E & UTM N & Description \\
\hline 06JEA65A & 64.7629 & -163.9439 & 550244 & 7182253 & Reddish-brown weathering, aphanitic to fine-grained, vesicular basalt. \\
\hline 06JEA249A & 64.8469 & -163.9826 & 548252 & 7191584 & $\begin{array}{l}\text { Brown weathering, black, fresh, basalt(?) dike with 3-5 percent feldspar } \\
\text { phenocrysts. Dike may trend 30(?) degrees (azimuth). }\end{array}$ \\
\hline 06JEA342A & 64.9062 & -164.1246 & 541425 & 7198095 & $\begin{array}{l}\text { Brown weathering basalt(?) dike. Possibly(?) has a strike of } 40 \text { degrees } \\
\text { (azimuth) and a dip of } 60 \text { degrees. }\end{array}$ \\
\hline 06JEA726A & 64.7352 & -163.9929 & 547962 & 7179127 & Basalt dike with 1- to 2-mm-diameter vesicles. \\
\hline 06JEA745A & 64.7779 & -163.8395 & 555180 & 7184012 & $\begin{array}{l}\text { Brown weathering, black, basalt(?) dike with 1- to 2-mm-diameter vesicles } \\
\text { filled with calcite, and phenocrysts of feathery, less than 1-mm-long } \\
\text { plagioclase. Also may contain biotite phenocrysts that are less than } 1 \mathrm{~mm} \text { in } \\
\text { diameter. }\end{array}$ \\
\hline 06LF348B & 64.793 & -163.8898 & 552759 & 7185659 & $\begin{array}{l}\text { Porphyritic andesite dike with } 10 \text { percent plagioclase and } 10 \text { percent pyroxene } \\
\text { phenocrysts in a very fine-grained matrix, which possibly contain biotite. }\end{array}$ \\
\hline 06LF529A & 64.7956 & -164.0936 & 543068 & 7185789 & $\begin{array}{l}\text { Coarse-grained granofels with green mica, } 10-15 \text { percent garnet, } 2 \text { percent } \\
\text { green amphibole, approximately } 30 \text { percent relict glaucophane, and } \\
\text { plagioclase porphyroblasts up to } 0.5 \text { inches in diameter. }\end{array}$ \\
\hline $\begin{array}{l}\text { 06LF586A } \\
\text { Nome } \\
\text { Quadrangle }\end{array}$ & 64.5924 & -165.2179 & 489569 & 7162856 & $\begin{array}{l}\text { Pale green granofels with } 10-20 \text { percent garnet (average } 1 \mathrm{~mm} \text { in diameter), } \\
30-40 \text { percent pyroxene(?) (pale green, blocky), } 15 \text { percent subhedral white } \\
\text { mica, and interstitial epidote and actinolite. }\end{array}$ \\
\hline 06MBW56A & 64.6348 & -163.9417 & 550588 & 7167982 & $\begin{array}{l}\text { Meta-calc-silicate rock within Casadepaga Schist that is less than } 1 \text { foot wide. } \\
\text { Light-brown weathering, white- to pale green-colored rock with indistinct, } \\
\text { slightly micaceous partings. Matrix is aphanitic to finely granular. Contains } \\
\text { randomly-oriented, euhedral, amphibole crystals up to } 3 \mathrm{~mm} \text { long. Also } \\
\text { contains crystals of plagioclase(?) and quartz. Rock is cut by sparse, brown, } \\
\text { secondary biotite and quartz veinlets, which are generally less than } 1 \mathrm{~mm} \text { in } \\
\text { width. }\end{array}$ \\
\hline
\end{tabular}


Table 3. (continued).

\begin{tabular}{|c|c|c|c|c|c|}
\hline Sample Number & Latitude & Longitude & UTM E & UTM N & Description \\
\hline 06MBW218A & 64.817 & -163.8627 & 553999 & 7188348 & $\begin{array}{l}\text { Lightly iron oxide-stained outcrop of grayish-brown weathering, massive, } \\
\text { jointed, equigranular gabbro dike. Dike contains approximately } 40 \text { percent } \\
\text { dark brown to black biotite and hornblende(?), and } 60 \text { percent white } \\
\text { plagioclase. Contains approximately } 1 \text { percent disseminated iron sulfide } \\
\text { (likely pyrrhotite since rock is magnetic). Weathering rind is } 1 \text { - to } 2 \text {-cm-thick; } \\
\text { submitted sample was taken from fresh pieces. Gabbro is fine-grained, with } \\
\text { crystals up to } 4 \mathrm{~mm} \text { in diameter, and an average grain size of } 1-\text { to } 2-\mathrm{mm} \text { in } \\
\text { diameter. Contains approximately } 3 \text { percent scattered, round amygdules filled } \\
\text { with calcite, which are up to } 1 \mathrm{~cm} \text { in diameter. Dike possibly trends } 250(?) \\
\text { degrees (azimuth). }\end{array}$ \\
\hline 06MBW299A & 64.7843 & -163.7849 & 557761 & 7184775 & $\begin{array}{l}\text { Lightly iron oxide-stained, white, very soft, quartz + very white mica-rich schist. } \\
\text { Potentially could have a felsic metaigneous protolith. Schist occurs as an } \\
\text { isolated, less than 3-foot-thick layer, surrounded by black graphitic quartzite. } \\
\text { Exposed in road cut along the south side of the Nome-Council road. }\end{array}$ \\
\hline 06MBW340A & 64.7857 & -164.1776 & 539092 & 7184629 & $\begin{array}{l}\text { Blocky, massive boulders of mafic granofels forming a } 10 \text {-foot-high knob in } \\
\text { the tundra. Brownish-gray weathering, green-, red-, and tan-colored, coarse- } \\
\text { grained (crystals up to } 0.5 \mathrm{~cm} \text { in diameter), equigranular, very poorly foliated, } \\
\text { garnet (red, approximately } 1-\text { to } 2-\mathrm{mm} \text {-diameter, approximately } 20 \text { percent) + } \\
\text { plagioclase (tan, approximately } 25-30 \text { percent) + amphibole (dark green, } \\
\text { euhedral, equant crystals, approximately } 55 \text { percent) mafic granofels. }\end{array}$ \\
\hline 06MBW393A & 64.7695 & -164.1091 & 542376 & 7182867 & $\begin{array}{l}\text { Sparse float chips of brown weathering, rounded (spheroidally weathered), } \\
\text { dark gray, fine-grained, plagioclase-porphyritic gabbro(?). Narrow dike. }\end{array}$ \\
\hline 06MBW462A & 64.7886 & -164.1815 & 538903 & 7184956 & $\begin{array}{l}\text { Metagraywacke(?). Blocky, gnarly weathering, massive, very indistinctly } \\
\text { foliated (rock is deformed, with irregular shear planes coated with dark green } \\
\text { chlorite(?)), anomalously very fine-grained, possibly composed of } 60 \text { percent } \\
\text { plagioclase and } 40 \text { percent, equant pyroxene(?) or secondary amphibole (after } \\
\text { pyroxene(?)). Trace iron oxide after sulfide. Unit forms a 10-foot-high knob } \\
\text { outcrop in tundra. }\end{array}$ \\
\hline 06RN247B & 64.5719 & -163.6769 & 563390 & 7161210 & $\begin{array}{l}\text { Bluff drill hole number } 5 \text {. Dike sample taken between } 22 \text { feet and } 45 \text { feet. } \\
\text { Dike is weathered, dark colored, fine grained, porphyritic. }\end{array}$ \\
\hline
\end{tabular}


Table 3. (continued).

\begin{tabular}{|c|c|c|c|c|c|}
\hline Sample Number & Latitude & Longitude & UTM E & UTM N & Description \\
\hline 06RN248A & 64.5728 & -163.6768 & 563390 & 7161310 & $\begin{array}{l}\text { Bluff drill hole number } 6 \text {. Dike sample taken between } 335 \text { feet and } 357 \text { feet. } \\
\text { Dike has occasional serpentine(?) and slickensides on sheared contacts. } \\
\text { Dike is dark, fine-grained, porphyritic(?), and has occasional disseminated } \\
\text { pyrite. }\end{array}$ \\
\hline 06RN248B & 64.5728 & -163.6768 & 563390 & 7161310 & $\begin{array}{l}\text { Bluff drill hole number } 6 \text {. Sample taken between } 416 \text { feet and } 446 \text { feet. Dike } \\
\text { has sheared contacts with marble host rock, inclusions of marble in the dike } \\
\text { along the contact, and the upper contact is approximately parallel with the } \\
\text { core axis. Calcite veins are also parallel with core axis. Sampled dike with 0- } \\
15 \text { percent, light greenish-brown clinopyroxene(?) phenocrysts, occasional } \\
\text { plagioclase(?) phenocrysts, and a very fine-grained matrix. }\end{array}$ \\
\hline 06RN294A & 64.8024 & -164.1462 & 540559 & 7186508 & $\begin{array}{l}\text { Altered feldspar porphyry intrusion. Possibly a gabbro(?). Intrusion contains } \\
\text { randomly oriented, 0.5- to 1-cm-diameter feldspars in a green groundmass } \\
\text { with minor remnant biotite(?). Not foliated, and found on top of knob. }\end{array}$ \\
\hline 06RN308A & 64.878 & -164.0555 & 544744 & 7195000 & $\begin{array}{l}\text { Mafic dike with biotite, olivine, and plagioclase phenocrysts that are less than } \\
\text { or equal to } 1 \mathrm{~mm} \text { in diameter. Phenocrysts make up } 30-60 \text { percent of the } \\
\text { rock, with the rest composed of dark, fine-grained groundmass. Dike is } \\
\text { approximately } 2-3 \text { meters wide, has a strike of approximately } 0 \text { degrees } \\
\text { (azimuth) and a dip of approximately } 90 \text { degrees. }\end{array}$ \\
\hline $\begin{array}{l}\text { 06RN595A } \\
\text { Nome } \\
\text { Quadrangle }\end{array}$ & 64.454 & -165.0467 & 497754 & 7147408 & $\begin{array}{l}\text { Orthogneiss with strongly gneissic fabric. Contains feldspar up to } 0.3 \mathrm{~cm} \text { in } \\
\text { diameter, approximately } 10-15 \text { percent chlorite (after biotite), and greater than } \\
50 \text { percent feldspar. }\end{array}$ \\
\hline 06Z114A & 64.7989 & -163.8921 & 552636 & 7186310 & $\begin{array}{l}\text { Mafic dike. Black, fine-grained, mafic rock consisting of hornblende, } \\
\text { plagioclase, biotite, and } 2-3 \text { percent fine-grained, disseminated pyrrhotite. } \\
\text { Contains } 1 \text { percent white calcite-filled amygdules that are approximately } 1 \mathrm{~cm} \\
\text { in diameter. Mafic minerals (biotite and hornblende) are about } 50 \text { percent of } \\
\text { the rock, and plagioclase is the other } 50 \text { percent. Possibly has a strike of } 40 \\
\text { degrees (azimuth). }\end{array}$ \\
\hline 06Z167A & 64.8421 & -163.941 & 550233 & 7191079 & $\begin{array}{l}\text { Basalt(?). Dark brown-weathering, black, fine-grained mafic igneous rock. } \\
\text { Plagioclase(?) crystals up to 1-mm-wide by } 3-\mathrm{mm} \text {-long. Trace disseminated } \\
\text { pyrite up to } 1 \mathrm{~mm} \text { in diameter. Aphanitic or very fine-grained matrix. Dike } \\
\text { strikes } 65 \text { degrees (azimuth) and is approximately } 5 \text { feet wide. }\end{array}$ \\
\hline
\end{tabular}


Table 3. (continued).

\begin{tabular}{|c|c|c|c|c|l|}
\hline Sample Number & Latitude & Longitude & UTM E & UTM N & \multicolumn{1}{|c|}{ Description } \\
\hline 06Z308B & 64.7917 & -163.9757 & 548681 & 7185441 & $\begin{array}{l}\text { Metamorphosed dike(?). Black rock interlayered with, or possibly cross- } \\
\text { cutting(?), pelitic schist. Aphanitic except for black amphibole(?). }\end{array}$ \\
\hline & & & & & $\begin{array}{l}\text { Biotite + white mica + plagioclase(?) semischist. Black and white, mottled, } \\
\text { fine-grained. One surface with 10 cm by 3 cm mass of black amphibole(?) } \\
\text { porphyroblasts(?) + biotite + chlorite + plagioclase. Possible metadike(?). }\end{array}$ \\
\hline
\end{tabular}


Table 4. Major-oxide, minor-oxide, and trace-element analyses for rocks collected in the Solomon and Nome Quadrangles, Seward Peninsula, Alaska.

\begin{tabular}{|c|c|c|c|c|c|c|c|c|c|c|c|c|c|c|c|c|c|c|c|c|}
\hline $\begin{array}{l}\text { Sample } \\
\text { Number }\end{array}$ & $\begin{array}{c}\mathrm{Al} 2 \mathrm{O} 3 \\
\% \\
\end{array}$ & $\begin{array}{c}\mathrm{BaO} \\
\% \\
\end{array}$ & $\begin{array}{c}\mathrm{CaO} \\
\% \\
\end{array}$ & $\begin{array}{c}\mathrm{Cr} 2 \mathrm{O} 3 \\
\% \\
\end{array}$ & $\begin{array}{c}\text { Fe2O3 } \\
\% \\
\end{array}$ & $\begin{array}{c}\mathrm{K} 2 \mathrm{O} \\
\% \\
\end{array}$ & $\begin{array}{c}\mathrm{MgO} \\
\% \\
\end{array}$ & $\begin{array}{c}\mathrm{MnO} \\
\% \\
\end{array}$ & $\begin{array}{c}\mathrm{Na2O} \\
\% \\
\end{array}$ & $\begin{array}{c}\text { P2O5 } \\
\% \\
\end{array}$ & $\begin{array}{c}\mathrm{SiO} 2 \\
\% \\
\end{array}$ & $\begin{array}{c}\mathrm{SrO} \\
\% \\
\end{array}$ & $\begin{array}{c}\mathrm{TiO} 2 \\
\% \\
\end{array}$ & $\begin{array}{c}\text { LOI } \\
\% \\
\end{array}$ & $\begin{array}{c}\text { Total } \\
\% \\
\end{array}$ & $\begin{array}{c}\mathrm{Nb} \\
\mathrm{ppm}\end{array}$ & $\begin{array}{c}\mathrm{Rb} \\
\mathrm{ppm}\end{array}$ & $\begin{array}{c}\mathrm{Sr} \\
\mathrm{ppm}\end{array}$ & $\begin{array}{c}\mathrm{Y} \\
\mathrm{ppm} \\
\end{array}$ & \begin{tabular}{|c|}
$\mathrm{Zr}$ \\
$\mathrm{ppm}$
\end{tabular} \\
\hline 06JEA65A & 5.67 & 0.20 & 8.83 & 0.01 & 8.29 & 2.41 & 4.08 & 0.15 & .64 & 0.53 & 45.56 & 0.09 & 1.45 & 7.99 & 98.89 & 13 & 71 & 912 & 28 & 292 \\
\hline 06JEA249A & .05 & .17 & .15 & 0.01 & 07 & .73 & 3.47 & 13 & 20 & .64 & 0.14 & .07 & 2.00 & 4.47 & 3.28 & 29 & 94 & 712 & 36 & 424 \\
\hline 06JEA342A & 5.84 & 0.18 & 4.12 & 0.01 & 9.04 & 3.02 & 2.37 & 0.1 & .14 & .97 & 52.42 & 0.04 & 2.31 & 4.86 & 8.42 & 37 & 77 & 389 & 53 & 545 \\
\hline 06JEA726A & 7.26 & 0.20 & 7.53 & 0.01 & 7.70 & 2.56 & 3.47 & .14 & .87 & .56 & 47.32 & 0.11 & 1.56 & 7.04 & 8.32 & 13 & 79 & 1090 & 28 & 308 \\
\hline 06JEA745A & & 0.21 & .28 & .02 & 8.77 & .71 & 4.09 & 14 & 94 & 59 & 8.80 & 07 & .60 & .11 & 09 & 19 & 104 & 723 & 32 & 347 \\
\hline 06LF348B & 5.94 & 0.17 & 7.22 & 0.01 & 8.39 & 1.60 & 4.53 & 0.16 & 2.82 & 55 & 46.59 & 0.09 & 1.47 & 3.97 & 98.51 & 13 & 38 & 938 & 29 & 302 \\
\hline 06LF529A & 7.51 & 0.01 & 1.79 & 0.06 & 9.10 & 0.13 & 7.67 & 0.14 & .76 & 0.06 & 45.85 & 0.03 & 0.82 & 3.86 & 98.79 & 3 & 4 & 299 & 14 & 58 \\
\hline 06LF586A & .30 & 0.01 & 1.15 & 0.01 & 12.05 & 0.27 & 6.61 & 8 & 31 & 09 & 46.82 & 0.02 & 1.09 & 1.69 & 09 & I & 10 & 180 & 20 & 88 \\
\hline 06MBW56A & 55 & 0.06 & 9.09 & 0.01 & 6.10 & 1.30 & 3.73 & 3 & 33 & 6 & 58.93 & 0.02 & 0.67 & 1.54 & 02 & 0 & 22 & 210 & 27 & 132 \\
\hline 06MBW218A & 16.78 & 0.21 & 6.87 & 0.01 & 9.17 & 2.08 & 4.50 & 0.15 & 3.29 & 0.59 & 47.64 & 0.08 & 1.45 & 5.96 & 98.77 & 13 & 65 & 764 & 28 & 307 \\
\hline 06MBW299A & 18.72 & 0.25 & 0.31 & 0.01 & 4.07 & 9 & 1.98 & 0.01 & 06 & 7 & 63.28 & 2 & 0.68 & .23 & 8.77 & 14 & 178 & 129 & 19 & 251 \\
\hline 06MBW340A & & 0.01 & 10.15 & 0.01 & 14.13 & & 5.64 & & 2 & & 48.86 & 3 & 1.50 & 2.23 & 9.10 & 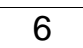 & $<2$ & 290 & 26 & 98 \\
\hline 06MBW393A & 16.28 & 0.10 & 6.27 & 0.03 & 8.98 & 1.72 & 5.54 & 0.26 & 2.60 & 0.62 & 47.81 & 0.05 & 2.29 & 5.66 & 98.21 & 23 & 50 & 515 & 44 & 413 \\
\hline 06MBW462A & 17.07 & 0.01 & 0.84 & 0.07 & 9.45 & 0.60 & 8.03 & .17 & 79 & 06 & 45.76 & 0.03 & 0.73 & .65 & 98.26 & 2 & 23 & 300 & 17 & 49 \\
\hline 06RN247B & 14.69 & 0.11 & 6.80 & 03 & 0 & 6 & 5 & & 8 & & 9 & 6 & 4 & 39 & 4 & 27 & 70 & 553 & 38 & 457 \\
\hline 06RN248A & 15.02 & 0.11 & 7.28 & 0.02 & 9.16 & 2.44 & 4.92 & 13 & 61 & 0 & 49.68 & 0.06 & 2.10 & 4.07 & 98.30 & 29 & 85 & 555 & 39 & 478 \\
\hline 06RN248B & 15.12 & 0.13 & 7.66 & 02 & 9.04 & 2.38 & 4.79 & 14 & 61 & 59 & 48.22 & 0.06 & 2.07 & .58 & 98.51 & 28 & 75 & 508 & 38 & 473 \\
\hline 06RN294A & 18.24 & 0.01 & 12.38 & 0.06 & 8.57 & 0 . & 7.65 & 2 & 60 & 6 & 45.42 & 0.03 & 0.69 & 3.51 & 98.42 & 3 & 2 & 248 & 13 & 47 \\
\hline 06RN308A & 3.84 & 0.16 & 7.12 & 0.04 & 7.35 & 2.06 & 6.65 & & 73 & & 49.01 & 0.07 & 1.29 & 7.71 & 98.63 & 21 & 98 & 683 & 28 & 275 \\
\hline 06RN595A & 14.12 & 0.01 & 0.51 & $<0.01$ & 3.09 & 0.41 & 1.31 & & 14 & & 70.82 & 0.01 & 0.46 & 1.22 & 8.21 & 21 & 19 & 108 & 39 & 292 \\
\hline 06Z114A & 16.83 & 0.20 & 6.51 & $<0.01$ & 9.18 & 2.55 & 4.56 & 5 & 8 & 5 & 48.13 & 0.08 & 1.54 & 4.81 & 98.27 & 14 & 90 & 777 & 31 & 358 \\
\hline 06Z167A & 14.93 & 0.16 & 5.79 & 0.01 & 8.67 & 2.78 & 3.21 & 0.14 & 2.88 & 0.89 & 51.97 & 0.07 & 2.09 & 5.09 & 98.67 & 33 & 80 & 680 & 45 & 513 \\
\hline 06Z308B & 20.79 & 0.01 & 1.01 & 0.01 & 6.95 & 0.19 & 6.00 & 0.02 & 6.35 & & 52.42 & 0.04 & 0.90 & 3.58 & 98.41 & 16 & 5 & 332 & 21 & 202 \\
\hline 06Z398B & 18.67 & 0.01 & 0.73 & 0.01 & 6.87 & 0.03 & 9.65 & 0.01 & 5.05 & 0.19 & 51.16 & 0.01 & 1.02 & 5.63 & 99.05 & 19 & $<2$ & 120 & 25 & 282 \\
\hline
\end{tabular}


Table 4. (continued)

\begin{tabular}{|c|cccccc|}
\hline $\begin{array}{c}\text { Sample } \\
\text { Number }\end{array}$ & $\begin{array}{c}\mathrm{Nb}^{*} \\
\mathrm{ppm}\end{array}$ & $\begin{array}{c}\mathrm{Rb}^{*} \\
\mathrm{ppm}\end{array}$ & $\begin{array}{c}\mathrm{Sr}^{*} \\
\mathrm{ppm}\end{array}$ & $\begin{array}{c}\mathrm{Y}^{*} \\
\mathrm{ppm}\end{array}$ & $\begin{array}{c}\mathrm{Zr}^{*} \\
\mathrm{ppm}\end{array}$ \\
\hline 06JEA65A & 7 & 69 & 899 & 33.6 & 255 \\
\hline 06JEA249A & 28.2 & 93 & 704 & 43.1 & 395 \\
\hline 06JEA342A & 42.8 & 77 & 384 & 61.4 & 530 \\
\hline 06JEA726A & - & - & - & - & - \\
\hline 06JEA745A & 16.9 & 102 & 704 & 37.5 & 315 \\
\hline 06LF348B & - & - & - & - & - \\
\hline 06LF529A & - & - & - & - & - \\
\hline 06LF586A & - & - & - & - & - \\
\hline 06MBW56A & - & - & - & - & - \\
\hline 06MBW218A & 10.4 & 63 & 752 & 35.5 & 283 \\
\hline 06MBW299A & - & - & - & - & - \\
\hline 06MBW340A & - & - & - & - & - \\
\hline 06MBW393A & 24 & 49 & 497 & 50.3 & 383 \\
\hline 06MBW462A & - & - & - & - & - \\
\hline 06RN247B & 29.2 & 69 & 543 & 43.7 & 432 \\
\hline 06RN248A & 30.1 & 81 & 530 & 45.1 & 438 \\
\hline 06RN248B & 30 & 75 & 601 & 45.8 & 448 \\
\hline 06RN294A & 2.3 & 3 & 241 & 13.7 & 44 \\
\hline 06RN308A & 18.6 & 95 & 661 & 32.1 & 246 \\
\hline 06RN595A & - & - & - & - & - \\
\hline 06Z114A & 11.8 & 89 & 761 & 36.5 & 327 \\
\hline 06Z167A & 33.4 & 79 & 671 & 53.1 & 481 \\
\hline 06Z308B & - & - & - & - & - \\
\hline 06Z398B & - & - & - & - & - \\
\hline
\end{tabular}

* = Pressed-pellet XRF analyses by UAF laboratory.

- = Not available 
Table 5. Detection limits for geochemical analyses. Analytical methods include: FA-AAS = Fire Assay-Atomic Absorption Spectroscopy, FA-GRAV = Fire Assay-Gravimetric Finish, ICP-AES = Inductively Coupled PlasmaAtomic Emission Spectroscopy, AAS = Atomic Absorption Spectroscopy. NOTE: * = possibly incomplete digestion dependent on mineralogy.

\begin{tabular}{|c|c|c|c|c|}
\hline Element & Units & $\begin{array}{c}\text { Lower } \\
\text { Detection } \\
\text { Limit }\end{array}$ & $\begin{array}{c}\text { Upper } \\
\text { Detection } \\
\text { Limit }\end{array}$ & $\begin{array}{c}\text { Analytical } \\
\text { Method }\end{array}$ \\
\hline $\mathrm{Au}$ & ppm & 0.005 & 10 & FA-AAS \\
\hline $\mathrm{Au}(+)$ & $\mathrm{ppm}$ & 0.05 & 1,000 & FA-GRAV \\
\hline $\mathrm{Ag}$ & ppm & 0.2 & 100 & ICP-AES \\
\hline$A I^{*}$ & percent & 0.01 & 25 & ICP-AES \\
\hline As & ppm & 2 & 10,000 & ICP-AES \\
\hline$B^{*}$ & $\mathrm{ppm}$ & 10 & 10,000 & ICP-AES \\
\hline $\mathrm{Ba}^{*}$ & ppm & 10 & 10,000 & ICP-AES \\
\hline $\mathrm{Be}^{*}$ & ppm & 0.5 & 1,000 & ICP-AES \\
\hline $\mathrm{Bi}$ & ppm & 2 & 10,000 & ICP-AES \\
\hline $\mathrm{Ca}^{*}$ & percent & 0.01 & 25 & ICP-AES \\
\hline $\mathrm{Cd}$ & ppm & 0.5 & 1,000 & ICP-AES \\
\hline Co & ppm & 1 & 10,000 & ICP-AES \\
\hline $\mathrm{Cr}^{*}$ & ppm & 1 & 10,000 & ICP-AES \\
\hline $\mathrm{Cu}$ & ppm & 1 & 10,000 & ICP-AES \\
\hline $\mathrm{Cu}(+)$ & $\%$ & 0.01 & 40 & AAS \\
\hline $\mathrm{Fe}$ & percent & 0.01 & 50 & ICP-AES \\
\hline $\mathrm{Ga}^{*}$ & ppm & 10 & 10,000 & ICP-AES \\
\hline $\mathrm{Hg}$ & ppm & 1 & 10,000 & ICP-AES \\
\hline $\mathrm{Hg}(+)$ & ppm & 0.01 & 100 & AAS \\
\hline $\mathrm{K}^{*}$ & percent & 0.01 & 10 & ICP-AES \\
\hline $\mathrm{La}^{*}$ & ppm & 10 & 10,000 & ICP-AES \\
\hline$M g^{\star}$ & percent & 0.01 & 25 & ICP-AES \\
\hline$M n$ & ppm & 5 & 50,000 & ICP-AES \\
\hline Mo & ppm & 1 & 10,000 & ICP-AES \\
\hline $\mathrm{Na}^{*}$ & percent & 0.01 & 10 & ICP-AES \\
\hline $\mathrm{Ni}$ & $\mathrm{ppm}$ & 1 & 10,000 & ICP-AES \\
\hline$P$ & ppm & 10 & 10,000 & ICP-AES \\
\hline $\mathrm{Pb}$ & ppm & 2 & 10,000 & ICP-AES \\
\hline $\mathrm{S}$ & percent & 0.01 & 10 & ICP-AES \\
\hline $\mathrm{Sb}$ & ppm & 2 & 10,000 & ICP-AES \\
\hline $\mathrm{Sc}^{*}$ & ppm & 1 & 10,000 & ICP-AES \\
\hline $\mathrm{Sr}^{\star}$ & $\mathrm{ppm}$ & 1 & 10,000 & ICP-AES \\
\hline $\mathrm{Ti}^{\star}$ & percent & 0.01 & 10 & ICP-AES \\
\hline$T l^{*}$ & ppm & 10 & 10,000 & ICP-AES \\
\hline $\mathrm{U}$ & ppm & 10 & 10,000 & ICP-AES \\
\hline V & ppm & 1 & 10,000 & ICP-AES \\
\hline$W^{*}$ & ppm & 10 & 10,000 & ICP-AES \\
\hline $\mathrm{Zn}$ & $\mathrm{ppm}$ & 2 & 10,000 & ICP-AES \\
\hline
\end{tabular}


Table 6. Detection limits for major-oxide, minor-oxide, and trace-element analyses. Analytical methods include: LBF-XRF = Lithium borate fusion and X-ray fluorescence spectroscopy, and PP-XRF = wavelength dispersive $\mathrm{X}$-ray fluorescence spectroscopy on a pressed pellet.

Note: $\mathrm{Fe}_{2} \mathrm{O}_{3}=$ total iron as $\mathrm{Fe}_{2} \mathrm{O}_{3} ; \mathrm{LOI}=$ loss on ignition.

\begin{tabular}{|c|c|c|c|c|}
\hline Element & Units & $\begin{array}{c}\text { Lower } \\
\text { Detection } \\
\text { Limit }\end{array}$ & $\begin{array}{c}\text { Upper } \\
\text { Detection } \\
\text { Limit }\end{array}$ & $\begin{array}{c}\text { Analytical } \\
\text { Method }\end{array}$ \\
\hline $\mathrm{Al}_{2} \mathrm{O}_{3}$ & percent & 0.01 & 100.00 & LBF-XRF \\
\hline $\mathrm{BaO}$ & percent & 0.01 & 100.00 & LBF-XRF \\
\hline $\mathrm{CaO}$ & percent & 0.01 & 100.00 & LBF-XRF \\
\hline $\mathrm{Cr}_{2} \mathrm{O}_{3}$ & percent & 0.01 & 100.00 & LBF-XRF \\
\hline $\mathrm{Fe}_{2} \mathrm{O}_{3}$ & percent & 0.01 & 100.00 & LBF-XRF \\
\hline $\mathrm{K}_{2} \mathrm{O}$ & percent & 0.01 & 100.00 & LBF-XRF \\
\hline $\mathrm{MgO}$ & percent & 0.01 & 100.00 & LBF-XRF \\
\hline $\mathrm{MnO}$ & percent & 0.01 & 100.00 & LBF-XRF \\
\hline $\mathrm{Na}_{2} \mathrm{O}$ & percent & 0.01 & 100.00 & LBF-XRF \\
\hline $\mathrm{P}_{2} \mathrm{O}_{5}$ & percent & 0.01 & 100.00 & LBF-XRF \\
\hline $\mathrm{SiO}_{2}$ & percent & 0.01 & 100.00 & LBF-XRF \\
\hline $\mathrm{SrO}$ & percent & 0.01 & 100.00 & LBF-XRF \\
\hline $\mathrm{TiO}_{2}$ & percent & 0.01 & 100.00 & LBF-XRF \\
\hline LOI & percent & 0.01 & 100.00 & LBF-XRF \\
\hline Total & percent & 0.01 & 105.00 & Calculation \\
\hline $\mathrm{Nb}$ & ppm & 2 & 10,000 & PP-XRF \\
\hline $\mathrm{Rb}$ & ppm & 2 & 10,000 & PP-XRF \\
\hline $\mathrm{Sr}$ & $\mathrm{ppm}$ & 2 & 10,000 & PP-XRF \\
\hline$Y$ & ppm & 2 & 10,000 & PP-XRF \\
\hline $\mathrm{Zr}$ & $\mathrm{ppm}$ & 2 & 10,000 & PP-XRF \\
\hline $\mathrm{Nb}^{*}$ & ppm & 1 & 100,000 & PP-XRF \\
\hline $\mathrm{Rb}^{*}$ & ppm & 0.5 & 100,000 & PP-XRF \\
\hline $\mathrm{Sr}^{\star}$ & ppm & 0.5 & 100,000 & PP-XRF \\
\hline$Y^{*}$ & $\mathrm{ppm}$ & 1 & 100,000 & PP-XRF \\
\hline$Z r^{\star}$ & ppm & 1 & 100,000 & PP-XRF \\
\hline
\end{tabular}

* = Pressed-pellet XRF analyses by UAF laboratory. 
Table 7. Location and description of rocks collected for non-carbonate carbon analyses in the Solomon Quadrangle, Seward Peninsula, Alaska. Carbon (non-carbonate) was analyzed by induction furnace pyrolysis following dilute acid digestion. The lower detection limit is 0.01 percent, and the upper detection limit is 50 percent carbon.

\begin{tabular}{|c|c|c|c|c|l|c|}
\hline Sample Number & Latitude & Longitude & UTM E & UTM N & \multicolumn{1}{c|}{ Description } & Non-carbonate Carbon (\%) \\
\hline & & & & & $\begin{array}{l}\text { Black, sooty, very fine-grained, finely foliated/laminated, graphitic } \\
\text { quartzite with 2-3 percent disseminated iron oxide after iron } \\
\text { sulfide (possibly pyrrhotite since the rock is relatively magnetic). }\end{array}$ & 1.08 \\
\hline 06MBW147A & 64.7770 & -164.0187 & 546663 & 7183772 & $\begin{array}{l}\text { Black, very sooty, graphite + quartz schist with fine, alternating } \\
\text { layers of quartz and graphite. }\end{array}$ \\
\hline 06LF442B & 64.8849 & -164.2139 & 537230 & 7195660 & 1.76 \\
\hline 06Z357A & 64.8352 & -164.3148 & 532511 & 7190070 & Silicified marble. & 0.07 \\
\hline
\end{tabular}

